\title{
Helioseismic diagnostics of solar dynamics in the near-surface layers
}

\author{
Dissertation \\ zur Erlangung des mathematisch-naturwissenschaftlichen Doktorgrades \\ "Doctor rerum naturalium" \\ der Georg-August-Universität Göttingen \\ im Promotionsstudiengang Physik \\ der Georg-August University School of Science (GAUSS)
}

\author{
vorgelegt von \\ Paul-Louis Poulier \\ aus Marseille, Frankreich
}

Göttingen, 2021 
Betreuungsausschuss

Prof. Dr. Laurent Gizon

Max-Planck-Institut für Sonnensystemforschung und

Institut für Astrophysik, Georg-August-Universität Göttingen, Göttingen, Deutschland

Dr. Thomas L. Duvall Jr.

Max-Planck-Institut für Sonnensystemforschung, Göttingen, Deutschland

PD Dr. Olga Shishkina

Max-Planck-Institut für Dynamik und Selbstorganisation, Göttingen, Deutschland

Mitglieder der Prüfungskommision

Referent: Prof. Dr. Laurent Gizon

Max-Planck-Institut für Sonnensystemforschung und

Institut für Astrophysik, Georg-August-Universität Göttingen, Göttingen, Deutschland

Korreferent: Prof. Dr. Thorsten Hohage

Institut für Numerische und Angewandte Mathematik, Georg-August-Universität Göttingen, Göttingen und

Max-Planck-Institut für Sonnensystemforschung, Göttingen, Deutschland

Weitere Mitglieder der Prüfungskommission:

Prof. Dr. Stefan Dreizler

Institut für Astrophysik, Georg-August-Universität Göttingen, Göttingen, Deutschland

Prof. Dr. Wolfram Kollatschny

Institut für Astrophysik, Georg-August-Universität Göttingen, Göttingen, Deutschland

PD Dr. Olga Shishkina

Max-Planck-Institut für Dynamik und Selbstorganisation, Göttingen, Deutschland

Prof. Dr. Andreas Tilgner

Institut für Geophysik, Georg-August-Universität Göttingen, Göttingen, Deutschland

Tag der mündlichen Prüfung: 23.02.2021 
(C) Paul-Louis Poulier

(c) This work is distributed under a
Creative Commons Attribution 3.0 License

Printed in Germany 



\section{Contents}

$\begin{array}{ll}\text { Summary } & 9\end{array}$

1 Introduction $\quad 11$

1.1 The interior of the $\operatorname{Sun} \ldots \ldots \ldots \ldots 11$

1.1.1 The solar structure . . . . . . . . . . . . . . 11

1.1.2 The solar oscillations . . . . . . . . . . . . . . 11

1.2 Acoustic waves and turbulence near the surface . . . . . . . . . . . 13

1.2.1 Small-scale turbulent convection near the solar surface . . . . . . 13

1.2.2 Acoustic wave propagation in a time-varying random medium . . 14

1.3 Large-scale flows in the Sun . . . . . . . . . . . . . . . . . 15

1.3.1 The global circulations: solar differential rotation and meridional circulation .................... 15

1.3.2 The time-varying component of the axisymmetric flows . . . . 16

1.4 Aims of this thesis . . . . . . . . . . . . . . . 18

1.4.1 Chapter 2: Acoustic waves and near-surface convection . . . . 18

1.4.2 Chapter 3: Medium-scale flows and solar activity . . . . . . . . 20

2 Acoustic wave propagation through solar granulation: Validity of effective$\begin{array}{lr}\text { medium theories, coda waves } & 21\end{array}$

2.1 Introduction . . . . . . . . . . . . . . . . . . . 22

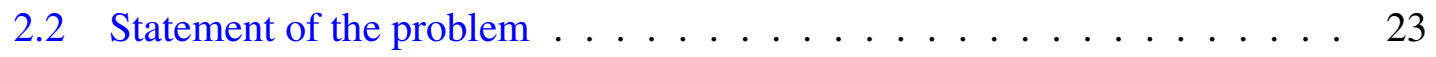

2.2.1 The random medium . . . . . . . . . . . . . 23

2.2 .2 The wave equation . . . . . . . . . . . . . . . 24

2.2.3 Characterizing the wave field .............. 26

2.3 Numerical methods . . . . . . . . . . . . . . . . . . . . . 27

2.3.1 Numerical scheme to solve for $\phi(x, t) \ldots \ldots$. . . . . . . . . 27

2.3.2 Numerical scheme to solve for $\tilde{\phi}(x, \omega) \ldots \ldots 29$

2.3.3 Measuring the attenuation . . . . . . . . . . . . . . 29

2.3.4 Measuring the effective wave speed . . . . . . . . . . . . . . . . 29

2.4 Effective medium theories . . . . . . . . . . . . . . . . . 29

2.5 Results and comparisons . . . . . . . . . . . . . . . 32

2.5.1 Coherent wave field . . . . . . . . . . . . . 32

2.5 .2 Attenuation . . . . . . . . . . . . . . . . . . . . 32

2.5.3 Effective wave speed .......................... 33

2.5.4 Variance of wave field . . . . . . . . . . . . . . . 34

2.5.5 Dependence on correlation time of the medium . . . . . . . . 36 
2.6 Discussion . . . . . . . . . . . . . . . . . . . 39

2.6.1 Accuracy of the theories . . . . . . . . . . . . 39

2.6.2 Validity of the frozen-medium approximation . . . . . . . . . 39

2.6.3 Detectability of coda waves . . . . . . . . . . . . . . . 40

2.7 Appendix . . . . . . . . . . . . . . . . . . . . 41

2.7.1 Keller approximation: Time-independent random medium . . . . 41

2.7.2 Keller approximation: Time-dependent random medium . . . . . 43

2.7.3 Second-order Born approximation . . . . . . . . . . . . . . . 44

2.7.4 Spatial homogenization . . . . . . . . . . . . . 45

2.7 .5 Ray approximation . . . . . . . . . . . . . . . 46

2.7.6 Comparing theories with numerical simulations in the limit $\epsilon \rightarrow 0 \quad 47$

3 Contribution of flows around active regions to the north-south helioseismic measurements 49

3.1 Introduction . . . . . . . . . . . . . . . . . . . 49

3.2 Horizontal flows from granulation tracking . . . . . . . . . 50

3.2 .1 Flow maps from LCT . . . . . . . . . . . . . . 50

3.2.2 Construction of background in CCD frame . . . . . . . . . 51

3.2 .3 Active-region flows ................. 51

3.3 Temporal variation of active-region flows . . . . . . . . . . . 55

3.4 Modeled helioseismic travel-time perturbations . . . . . . . . . . 56

3.4.1 Computation of travel-time perturbations . . . . . . . . . 56

3.4.2 Effect of masking travel-time perturbations inside magnetic regions ........................... 58

3.5 Extension to May 1996-April $2019 \ldots \ldots$. . . . . . . . . . . . . . . . . . . . 60

3.6 Comparison with helioseismic measurements . . . . . . . . . . . 62

3.7 Summary and discussion . . . . . . . . . . . . . . . . . . . 64

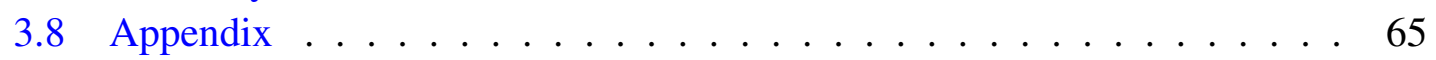

3.8.1 Definition of contour . . . . . . . . . . . . 65

3.8.2 Temporal variation of the background flows $\ldots \ldots . . \ldots 67$

3.8.3 3D Born sensitivity kernels . . . . . . . . . . . . 67

4 Discussion $\quad \mathbf{7 1}$

4.1 Properties of flows around the average active region . . . . . . . . . 71

4.1.1 LCT data show a retrograde flow in the leading polarity . . . . 71

4.1.2 Dependence of the flows on the total unsigned magnetic flux . . . 71

4.1.3 Effect of the flows around the selected active regions on the solarcycle variation of the meridional flow . . . . . . . . . . 72

4.1 .4 Conclusion of the analysis . . . . . . . . . . . . . 74

4.2 Conclusion and outlook . . . . . . . . . . . . . . . 75

4.2.1 Propagation of acoustic waves through turbulent convection . . . 75

4.2.2 Near-surface inflows around active regions . . . . . . . . . . 75 
A Derivation of the second-order Born approximation for a slab of frozen random medium

Publications

Acknowledgements 



\section{Summary}

This thesis proposes to develop new helioseismic diagnostics of solar near-surface flows. In a first part, we discuss the interaction of solar seismic waves (sound waves) with small-scale turbulent convection. The treatment of the effect of turbulent convection on waves is challenging because it involves all spatial and temporal scales. We consider several effective-medium approximations used to describe the propagation of acoustic waves through solar granulation and compare these approximations with numerical simulations. For large-amplitude perturbations, we find that the Keller approximation is best suited to estimate the effective wave speed and attenuation. While the temporal evolution of granulation may be ignored when estimating the effective wave speed, it must be taken into account in the computation of the attenuation. In addition, late arrival waves due to multiple scattering (coda waves) are seen in the simulations in the variance of the wave field. This work will help improve our understanding of the physics of the modes of solar oscillations, and contributes to the interpretation of global and local helioseismology observations.

In a second part, we study near-surface local flows around solar active regions. These active-region inflows are important because they contribute to the observed solar-cycle changes in the longitudinal average of the solar meridional circulation. Using measurements of the inflows with a granulation tracking method and a model for their depth dependence, we solve the forward problem of time-distance helioseismology to estimate their contribution to the observed helioseismic travel times. In the granulation-tracking maps, the inflows contribute up to $\pm 7 \mathrm{~m} / \mathrm{s}$ to the surface meridional flow, which is about $50 \%$ of its amplitude. We find however that the travel-time perturbations associated with near-surface active-region flows do not explain in full the solar-cycle variations observed in the seismic data. This work paves the way for correcting the travel times for the nearsurface flows, in order to probe the cycle variations of the meridional flow at depth. 



\section{Introduction}

\subsection{The interior of the Sun}

\subsubsection{The solar structure}

The Sun is a 4.5-billion-year-old rotating G-type star lying on the main sequence, with a radius $R_{\odot}$ of $696 \mathrm{Mm}$. It mostly consists of hydrogen and helium. Its interior structure can be divided up into several distinct regions.

The inner core extends from the center to about $25 \%$ of the solar radius $R_{\odot}$. There, hydrogen is transformed into helium through nuclear fusion at temperatures up to 15 million degrees. The resulting pressure gradient balances gravity, maintaining hydrostatic equilibrium. Up to $0.73 R_{\odot}$, the radial temperature gradient of the medium is subadiabatic, meaning that convection cannot take place. Radiation transports the energy from the core outward through this region: it is the radiative zone. At a depth of $0.73 R_{\odot}$, the temperature gradient becomes slightly super-adiabatic, such that convection becomes the dominant mechanism of energy transport (Christensen-Dalsgaard et al. 1991). At the top of the convection zone, due to a drop of the density by several orders of magnitude, the opacity decreases enough to let photons escape. The location of this opacity drop is called the photosphere and is a few hundred kilometers thick. The solar spectrum is roughly that of a black body with a temperature of about $5800 \mathrm{~K}$. The solar atmosphere, above the surface, consists of the chromosphere, the transition region and the corona, at which point the temperature has risen again up to $2 \times 10^{6} \mathrm{~K}$, for reasons that are still debated.

\subsubsection{The solar oscillations}

Leighton et al. (1962) first observed that the velocity field at the solar surface oscillates with a period of about five minutes, corresponding to a frequency of about $3 \mathrm{mHz}$. It was then hypothesized (e.g., Ulrich 1970, Leibacher and Stein 1971) that this signal comes from the superposition of standing waves trapped inside cavities below the photosphere. Deubner (1975) then observed for the first time the concentration of oscillatory power along ridges in frequency - horizontal wave number space. Such a power spectrum is presented in Fig. 1.1.

The oscillations are stochastically excited by the near-surface turbulent convection (e.g., Goldreich and Keeley 1977, Houdek 2006, Houdek and Dupret 2015). There exist three types of modes. The acoustic $(p)$ modes are sound waves, for which the restoring force is pressure. These waves propagate between the near-surface, where most of them are reflected, and an inner turning point corresponding to where the horizontal phase speed 
becomes equal to the local sound speed (Leibacher and Stein 1981, Duvall 1982). Because the sound speed increases with depth, the larger the phase speed is, the deeper the modes propagate. The power of these oscillations peaks at a frequency of about $3 \mathrm{mHz}$. The second type of waves consists of the surface gravity, also known as $f$ modes. They propagate near the surface like riddles on water, and their restoring force is buoyancy. They can be used for example to probe near-surface convection or measure rotation. Finally, the gravity $(g)$ modes are trapped inside the radiative zone and are driven by buoyancy fluctuations. Both acoustic and surface gravity modes are visible in the power spectrum of both the continuum intensity and the line-of-sight velocity measurements of the solar surface, superimposed to the surface convection power spectrum. However, because the $g$ modes are evanescent in the convection zone, their direct observation is difficult and still debated (e.g., Fossat et al. 2017, Schunker et al. 2018).

The observed solar oscillation modes, called normal modes, oscillate at frequencies called eigenfrequencies. They can be decomposed using three quantum numbers: $n$, the number of radial nodes; $l$, the number of surface nodes; and $m \in[-l,+l]$ the number of equator-crossing nodes. $l$ is related to the horizontal wave number $k_{h}$ by $k_{h}=\sqrt{l(l+1)} / R_{\odot}$.

Helioseismology studies the structure and flows inside the Sun by using the information contained in its oscillations. It makes use of continuous and high-resolution observations coming from ground-based networks like the Global Oscillation Network Group (GONG, Harvey et al. (1996)) or space telescopes like the Michelson Doppler Imager (MDI, Scherrer et al. (1995)) onboard the SOlar and Heliospheric Observatory (SOHO) and the Heliosesmic and Magnetic Imager (HMI, Scherrer et al. (2012)) onboard the Solar Dynamics Observatory (SDO). These instruments observe(d) the solar disk in some spectral line (HMI for instance uses the Fe I line at $617.3 \mathrm{~nm}$ ). The forward problem in helioseismology consists in retrieving a change in the observable, generally the lineof-sight Doppler velocity, due to a perturbation from a reference medium. The inverse problem aims at retrieving the departure from this background medium using the observables. Basu (2010) for instance provides a summary of the inversion methods used in helioseismology. Global helioseismology (e.g., Christensen-Dalsgaard 2002) is the study of the modes of the Sun that tell about its global structure, with some of the main results being the determination of the depth and latitudinal dependence of the solar rotation (e.g., Duvall et al. 1986, Libbrecht 1989, Schou et al. 1998) and that of the sound speed profile (Christensen-Dalsgaard et al. 1985). Local helioseismology on the other hand, is used to probe the local structure on smaller areas. Several techniques can be used, among which is time-distance helioseismology. First formulated by Duvall et al. (1993), it involves the cross-correlation of the Doppler signal at any two points of the solar surface, as a proxy for the wave packets traveling between these points. One wave packet goes directly from the first point to the second point (first skip), while others bounce at the surface before reaching destination. The greater the separation distance, the deeper the first-skip wave packet reaches. One can measure the travel time of the wave packet in both directions, which gives indications about the flows along or against the wave field (e.g., Burston et al. 2015). For a review of local helioseismology in general, see for example Gizon and Birch (2005) and Gizon et al. (2010). 


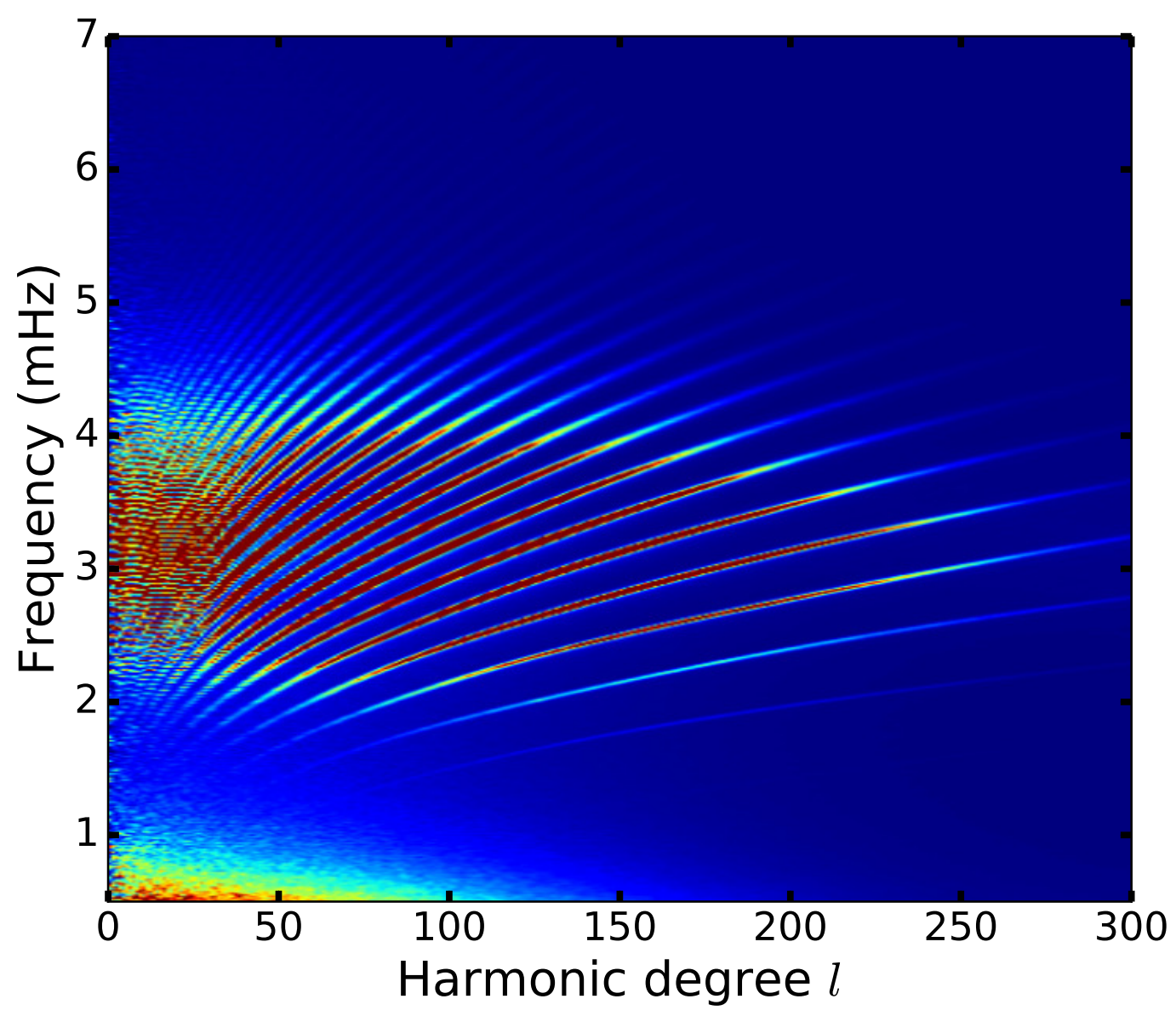

Figure 1.1: $m$-averaged power spectrum of SDO/HMI Dopplergrams. Red corresponds to high power. One day of medium- $l$ data was taken.

\subsection{Acoustic waves and turbulence near the surface}

\subsubsection{Small-scale turbulent convection near the solar surface}

Near the solar surface, in the photosphere, different scales of turbulent convection have been observed (Rieutord et al. 2010). These scales are selected by the constraint of mass conservation on upward and downward motions. The most easily identifiable pattern is granulation, mostly driven by radiative cooling from the surface (Nordlund et al. 2009). It consists of a myriad of bright cells covering the surface and separated by darker lines called intergranular lanes. Each cell is made of a hot parcel of gas, rising from below due to convective instability. The upward motion is essentially isentropic. The plasma then cools down at the surface and falls back inward in the intergranular lanes due to gravity. There, the downdrafts receive entropy by mixing with the upflows, so that the entropy difference between the former and the latter decreases with depth. Granulation is visible both on intensity images (due to the temperature variations) and on Doppler images (due to the line-of-sight velocity variations). The spatial size of a granule is typically 1 to 2 
$\operatorname{Mm}(1 \mathrm{Mm}=1000 \mathrm{~km})$, while its life time is of the order of ten minutes. The flows typically have velocities of 1 to $2 \mathrm{~km} / \mathrm{s}$, which is 15 to $30 \%$ of the local sound speed (about $7 \mathrm{~km} / \mathrm{s}$ ).

Supergranulation on the other hand, first discovered by Hart (1954), is a larger-scale convective pattern, with a life time of 1.5 days and a size of roughly $30 \mathrm{Mm}$ (Leighton et al. 1962, Rieutord et al. 2008, Hirzberger et al. 2008). The flows have an rms value of about $300 \mathrm{~m} / \mathrm{s}$, and are mostly horizontal, which explains why one sees them best far from disk center on Dopplergrams. They are however barely detectable in intensity observations. Whether supergranulation is convectively driven, has been debated for years (e.g., Rieutord and Rincon 2010, Rincon and Rieutord 2018). An extensive review of solar surface convection, from the observational and computational sides, can be found in Nordlund et al. (2009).

\subsubsection{Acoustic wave propagation in a time-varying random medium}

In the context of helioseismology and more broadly asteroseismology, understanding the pulsations of the star is paramount to infer properties such as radius, mass, etc. However, for the Sun already, there is a discrepancy between standard solar model predictions of the eigenfrequencies of the modes and the observations (e.g., Libbrecht et al. 1990, Gough and Toomre 1991, Christensen-Dalsgaard et al. 1996). The differences increase with frequency and scale roughly as the mode mass, which is the ratio of the mode energy to the square of the surface velocity (Libbrecht and Woodard 1991). This implies that the cause of this difference lies in near-surface regions, far above the turning point of the waves (Balmforth 1992b). This is why it is called the surface effect, which translates for example by a departure of the observed eigenfrequency from a standard model by about $-10 \mu \mathrm{Hz}$ at $3.5 \mathrm{mHz}$.

The discrepancies stem from an imperfect modeling of the superadiabatic shallow layer below the surface by the mixing-length theory used in standard solar (stellar) models. The surface effect can be decomposed into a model, or structural, effect, because the structure of the shallow subsurface is not well represented, and a modal effect, because the fluid equations do not hold anymore (non-adiabatic effects become important).

Brown (1984) and Delache and Fossat (1988) for example, investigated analytically the scattering effect of velocity fluctuations in the upper convection zone on the eigenfrequencies of the $p$ modes. Rosenthal et al. (1999) suggested to patch an averaged 3D numerical simulation of the near-surface convection on top of a standard solar model, in order to account for the structural term. They also tried to address the difficult question of how to include the turbulent pressure term in averaged fluid equations, in order to account for the mode physics. In addition, they made the assumption that the time scales of convective motions are greater than the period of the waves, while noting this is not valid for the Sun. Sonoi et al. (2015), Ball et al. (2016) for example, followed up on this idea and managed to significantly reduce the discrepancy. Houdek et al. (2017), in a way similar to Balmforth (1992a), and making use of a non-local mixing length theory (Gough 1977), tackled the modal effect and brought further improvement, bringing the difference down to around $2 \mu \mathrm{Hz}$. Schou and Birch (2020) numerically estimated the eigenfunctions in 3D simulations and found frequency differences consistent with Houdek et al. (2017). Another (practical) approach to resolve the discrepancy consists in parametrizing the surface 
term, regardless of the underlying physics, in order to generalize to other stars (Sonoi et al. 2015, Ball and Gizon 2017). One of the challenges in modeling the surface effect is to determine which approximations can be used when trying to include the mode physics. In particular, the solar turbulent convection has time scales that are comparable to the periods of the waves that propagate in it, requiring a priori a time-dependent treatment. Gough (1977), Unno (1967), Balmforth (1992c), to cite a few, developed mixing-length theories including the time dependence. Roth and Stix $(2001,2003)$ investigated theoretically the coupling of time-varying velocity fields with acoustic oscillations. More recently, Houdek et al. (2017) and Schou and Birch (2020) included time-dependent convection in their models.

In addition to the surface effect, the incoherent wave scattering contributes to the damping of the acoustic modes by mixing modes of different degrees (Bogdan 1989, Goldreich and Murray 1994). Goldreich and Murray (1994) found the associated damping rates to be in good qualitative agreement with the linewidths measured by, for example, Libbrecht (1988) and Libbrecht and Woodard (1991). It is not however the only mechanism responsible for the damping (e.g., Balmforth 1992a, Houdek et al. 1999).

It should be noted that a similar effect is observed on the $f$ modes. They experience a frequency shift and a mode damping because of multiple scattering of the waves by the surface turbulent horizontal flows (see for example Murawski and Roberts (1993a,b), Murawski et al. (1998), Duvall et al. (1998), Mȩdrek et al. (1999)).

\subsection{Large-scale flows in the Sun}

\subsubsection{The global circulations: solar differential rotation and merid- ional circulation}

There are two axisymmetric flows in the Sun. The first one is the solar differential rotation. The Sun rotates with a period of about 25 days at the equator $(\simeq 2 \mathrm{~km} / \mathrm{s})$, and more than 30 days near the poles. The rotation also depends on depth, as has been shown by global helioseismology using the frequency splittings of $p$ modes (Duvall and Harvey 1984, Duvall et al. 1984, 1986, Thompson et al. 1996, Schou et al. 1998, Larson and Schou 2018). It increases from the surface inward in the outer 5\% of the Sun (e.g., Barekat et al. 2014), and decreases thereafter. At $0.95 R_{\odot}$ is the so-called near-surface shear layer. The tachocline, at about $0.7 R_{\odot}$, is another shear layer below which the rotation becomes that of a solid body (e.g., Christensen-Dalsgaard and Schou 1988, Brown et al. 1989). The surface rotation rate has been measured via different methods: direct Doppler velocity measurements (Howard and Labonte 1980), small magnetic feature tracking (Howard et al. 1990), Doppler feature tracking (Snodgrass and Ulrich 1990), supergranular waves (Schou 2003). The answer varies slightly depending on the method, which probably comes as a consequence of the depth dependence. A review of solar rotation can be found in Thompson et al. (2003).

The second axisymmetric flow is the meridional circulation. It has been measured by different methods. A consensus was reached that it is a poleward flow at the surface in both hemispheres, with an amplitude of roughly $15 \mathrm{~m} / \mathrm{s}$ (Duvall 1979, Giles et al. 1997, Zhao and Kosovichev 2004, Hathaway 2012, Komm et al. 2018). The flow must return 
at some depth toward the equator because of mass conservation. The depth at which the return flow starts is still debated, as well as whether there is one cell (Hathaway 2012, Mandal et al. 2018, Gizon et al. 2020) or multiple ones (Zhao et al. 2013, Chen and Zhao 2017) (see also Böning et al. (2017)). The meridional flow is much weaker than supergranulation or differential rotation (about $200 \mathrm{~m} / \mathrm{s}$ ), so random noise and a variety of systematics make its determination much more difficult. This circulation is responsible for magnetic flux transport at the surface (advection of magnetic elements to the poles in the Babcock-Leighton mechanism of the solar dynamo) and is responsible in some dynamo models for the emergence of sunspots closer to the equator as the cycle progresses, therefore its measurement is key to understand the dynamo (Sheeley 1992, Dikpati and Gilman 2006, 2009, Gizon et al. 2020).

The meridional circulation results from an imperfect balance between two driving forces that result from the differential rotation and from an entropy gradient (Kitchatinov 2011, 2016). The differential rotation is sustained by the transport of angular momentum by turbulent convection via the Coriolis force (Reynolds stresses). This transport operates inward at small depths and equatorward at greater depths. Rotation is therefore not constant on cylinders aligned with the rotation axis, creating a torque in the sense of a surface poleward flow (Thompson et al. 2003); this is called the (non-conservative) centrifugal force or gyroscopic pumping. On the other hand, the Coriolis force inhibits the convective heat flux at low latitudes, making the poles slightly warmer than the equator. The induced entropy gradient creates, via the buoyancy force, a circulation that is equatorward at the surface; this is called the thermal forcing, or baroclinic driver, because the entropy gradient is related to a misalignment of the isosurfaces of pressure and density. The small difference between the two forces is responsible for the observed meridional circulation.

\subsubsection{The time-varying component of the axisymmetric flows}

These large-scale flows exhibit a time dependence. The time-varying part of the differential rotation is called torsional oscillations, whose amplitude is a few $\mathrm{m} / \mathrm{s}$ (Howard and Labonte 1980, Gizon 2003, 2004, Zhao and Kosovichev 2004). It looks like a shear flow at the latitude of mean activity, with a faster-rotating region on the equatorward side and a slower-rotating one on the poleward side. As the cycle progresses, it drifts toward the equator along with the magnetic activity, as has been seen by global (Kosovichev and Schou 1997, Schou et al. 1998, Schou 1999, Antia and Basu 2000) and local helioseismology (Basu and Antia 2000, Haber et al. 2000, Gizon 2003, Komm et al. 2004, 2014). Another shear region is present at high latitudes, that migrates toward the poles (Toomre et al. 2000, Antia and Basu 2001, Komm et al. 2014). It coincides with the high-latitude activity branch and seems to confirm that the zonal flows are connected to the regions of magnetic activity. The torsional oscillations are seen through much of the depth of the convection zone (e.g., Howe et al. 2000, Vorontsov et al. 2002).

The meridional circulation varies too, with time residuals also reaching a few $\mathrm{m} / \mathrm{s}$ as shown by magnetic-feature tracking (Komm et al. 1993, Komm 1994, Meunier 1999) and helioseismology (Chou and Dai 2001, Gizon 2003, 2004, Zhao and Kosovichev 2004, Zhao et al. 2014, Komm et al. 2015). The time residuals present a pattern similar to surface inflows converging toward the activity belt at low to mid-latitudes, also drifting 


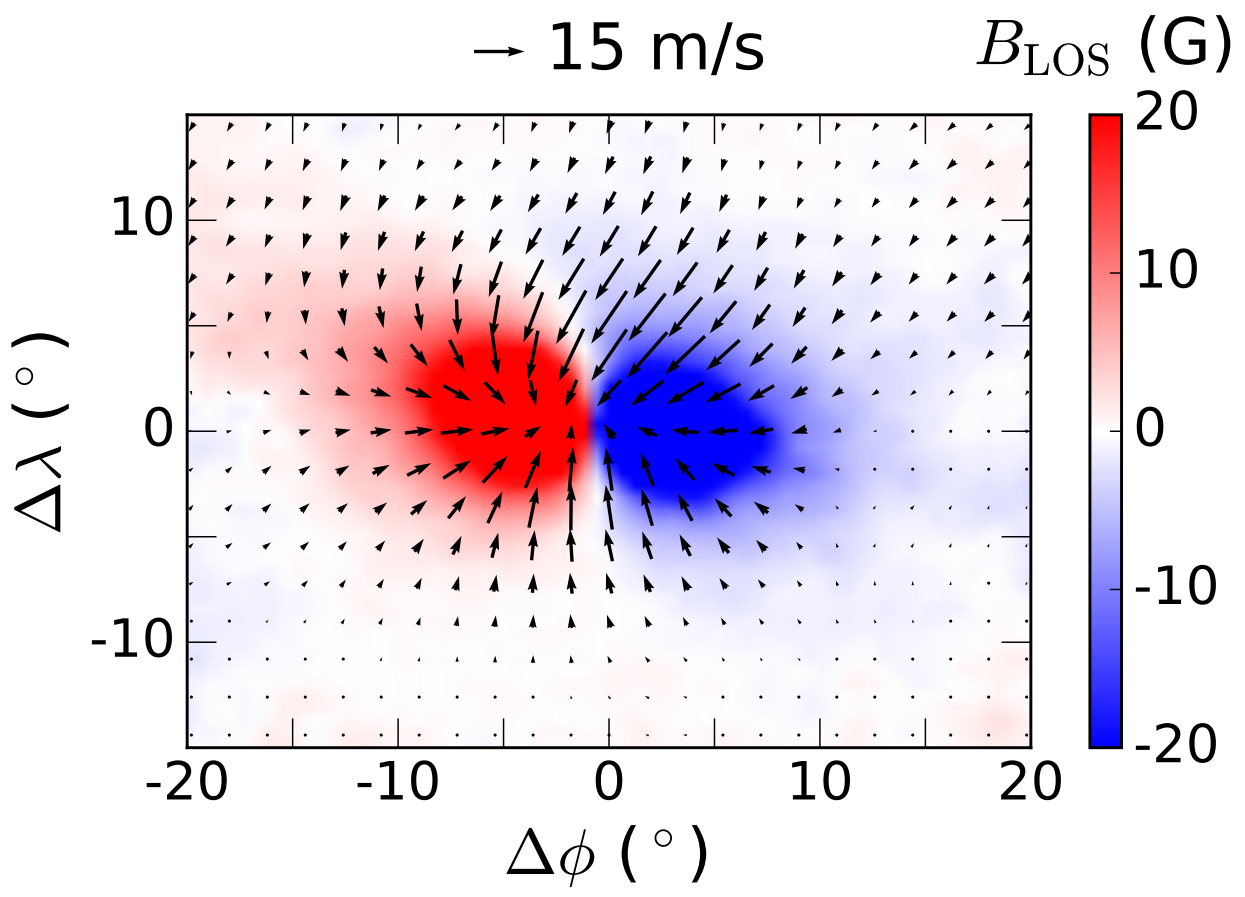

Figure 1.2: Inflows around an average active region, measured via the local correlation tracking of solar granulation on SDO/HMI intensity maps (see Subsection 1.4.2). $\Delta \lambda$ and $\Delta \phi$ are the angular distances to the center of the active region, respectively in the latitudinal and in the longitudinal directions. $\Delta \lambda>0^{\circ}$ is poleward, $\Delta \phi>0^{\circ}$ is prograde. The flows have been smoothed with a 2D Gaussian of $\sigma=2^{\circ}$. The flows at the center of each polarity have been removed for clarity. The line-of-sight magnetic field has been overplotted.

with time toward the equator. Surface inflows toward active regions have been observed for instance by Gizon et al. (2001), Hindman et al. (2003), Haber et al. (2004), Hindman et al. (2009), Braun (2019), with amplitudes reaching at the surface $50-100 \mathrm{~m} / \mathrm{s}$ for single regions and $10-20 \mathrm{~m} / \mathrm{s}$ on average. Helioseismology has provided different answers regarding the depth profile of the flows (e.g., Chou and Dai 2001, Haber et al. 2004, Zhao and Kosovichev 2004, Chou and Ladenkov 2005, Komm et al. 2020). In general, it is agreed that the inflows are localized in the upper $20 \mathrm{Mm}$ and become outflows thereafter. Fig. 1.2 shows the inflows around an average active region near the surface.

An open question is whether the inflows around the activity belt explain the totality of the time variation of the meridional circulation. Gizon (2003) found that they can explain it at the level of a few m/s. Cameron and Schüssler (2010) found a similar conclusion using a semi-empirical model of inflows. However, González Hernández et al. (2008) contested this result, finding in particular an inflow pattern even when excluding the surroundings of the active regions from their measurements.

Several mechanisms have been proposed to explain these flows. A possible driver is the feedback from the magnetic field via the Lorentz force (Yoshimura 1981, Schuessler 
1981). Schuessler (1981) showed that theoeretically, the kinetic energy provided by the Lorentz force in the toroidal direction is enough to quickly drive torsional oscillations of a few m/s. However, (Rempel 2006, 2007), using a dynamic flux-transport dynamo model, found that it could only account for the poleward high-latitude branch of the torsional oscillations, meaning that other processes had to play a role. They found that a thermal origin could explain the equatorward part. This process was initially described by Spruit (2003), who proposed that the enhanced radiative cooling by the small-scale magnetic field in the activity belts would create a region of slightly lower pressure. The Coriolis force would then drive a geostrophic zonal flow, just like on Earth. Inflows toward the active regions are predicted as a natural side effect of this theory. The flow is indeed not purely geostrophic because of viscosity effects. The generated Ekmann spiral then provides a poloidal flow of amplitude similar to that of the toroidal flow. The idea of this cyclonic motion is supported by helioseismic observations (e.g., Hindman et al. 2009, Komm et al. 2015), while some shortcomings remain, e.g. the fact that torsional oscillations precede the emergence of the magnetic field. Gizon and Rempel (2008) followed up on the model by Rempel (2006) and found that they could explain both torsional oscillations and meridional circulation variations, with values consistent with observations near the surface, while inconsistencies still remained at greater depths.

\subsection{Aims of this thesis}

This work focuses on the dynamics of the solar surface, namely variations of the solar medium or solar flows occurring near the surface on time scales smaller than, say, a solar cycle. The main question is how to average time-varying quantities to provide a meaningful background medium or background flow. In the first case, we look at time scales of the order of a few minutes (small-scale turbulent convection) and study how to find an effective medium for the propagation of acoustic waves through solar granulation. In the second case, we look at time scales of days to months, namely the active-region inflows that modulate the meridional flow; the goal is to find a background meridional flow that does not contain an imprint of the inflows and, conversely, to better constrain the impact of the inflows on the solar-cycle variation of the meridional flow.

\subsubsection{Chapter 2: Acoustic waves and near-surface convection}

The first paper included here tries to tackle the issue of the interaction between solar granulation and acoustic waves. More precisely, only the action of the medium on the waves is studied, while the back-reaction is neglected. Using second-order finite differences in an explicit scheme, we model numerically the propagation of acoustic modes through a one-dimensional medium that has been perturbed. The wave equation that we use is that of Gizon et al. (2017). We compare a certain number of wave scattering theories to the numerical (true) solutions, showing that a theoretical prediction of the scattering effect of the turbulent medium on solar acoustic waves is possible.

The study is based on the fact that solar granulation can be seen as a random process that modifies the background medium in which the solar acoustic waves propagate (quantities like temperature, density and small-scale velocity fields vary randomly). There- 
fore, the propagation of the waves is modified through (multiple) scattering. In helioseismology, in order to lower the noise level, it is common to average the observables (for instance, travel times for time-distance helioseismology) over time and space, that is over realizations of the turbulent medium (whether the realizations are independent is controlled by the correlation scales of the medium). Since each wave goes through a realization, it is itself part of a random process. It makes sense then to look at how the average, coherent wave field propagates. We also look at the variance of the wave field that contains information about the part that is scattered multiple times (coda waves).

Numerically, we use a Monte-Carlo approach by generating many realizations of a random medium with given statistical properties, and we propagate an acoustic wave packet in each of them before averaging the solutions over the realizations. We also derive the theoretical average wave field for a number of approximations down to the second order in the perturbation (the first-order correction is zero on average). One of them is the Born approximation, which gives a development of the perturbed wave field as a function of the perturbation to a background state, provided that the perturbation is "small" enough, as well as the ratio of the wavelength over the typical spatial size of the perturbation. The Keller approximation (Keller 1964) is based on a similar idea, but instead of providing a development of the solution, it rather looks at the equation satisfied by the average wave field. The homogenization theory (e.g., Hanasoge et al. 2013) and the geometrical optics (e.g., Tatarskii 1971) work for wavelengths respectively greater and smaller than the size of the perturbation. Unluckily, in the case of solar granulation and acoustic waves, the two quantities are comparable, meaning that we are in between the two regimes.

Both the numerical simulations and the theories show that the amplitude of the average wave field is reduced, because of the loss of coherent signal along the randomlyscattered path. Moreover, the effective path length browsed by the wave is increased by the multiple scattering, which decreases the effective wave speed. These two effects are qualitatively compatible with theoretical predictions and observations of the surface effect and the damping of the $p$ modes but we do not investigate this aspect further because our setup is very simplified compared to the true solar medium. We find that the Keller theory works best to describe the propagation of the coherent wave field, followed by the Born approximation that is valid for weaker perturbations or smaller distances of propagation. We derive the Keller solution in a time-dependent medium so that we can check the accuracy of the theory for different correlation times of the medium. We find, perhaps surprisingly, that one can use only a snapshot of granulation and still retrieve the effective wave speed, while the attenuation is underestimated. Considering a frozen medium would greatly simplify the theoretical and numerical study of wave scattering because frequencies would be uncoupled and the problem could be tackled in frequency domain via the Helmholtz equation. Finally, we show that the statistical variance of the wave field exhibits coda waves that are invisible in the coherent wave field. The coda waves are an indication of multiple scattering and have yet to be detected in the Sun, although they are commonly studied in terrestrial seismology (e.g., Aki 1969, Aki and Chouet 1975). 


\subsubsection{Chapter 3: Medium-scale flows and solar activity}

The second work presented in this thesis is observational. The motivation is a better measurement of the surface meridional flow and of its time-varying component. It would allow one to improve the inversions of the depth profile. A better knowledge of the meridional circulation would in turn help constrain the solar dynamo models. In this work, we check whether the surface inflows around regions of magnetic activity are responsible for the totality of the solar-cycle modulation of the meridional flow.

To do so, we use nine years of already-generated data from local correlation tracking of granulation, which is a method to retrieve surface flows (e.g., November and Simon 1988). The idea of the method is to track a certain type of features (granulation, supergranulation, magnetic features) over time. It is done by correlating in space (in two dimensions $x$ and $y$ ) pairs of small patches of observation maps (for example intensity maps or magnetograms) separated by a time lag $\Delta t$; for each patch, the maximal correlation arises for some distances $\left(d_{x}, d_{y}\right)$ (in both directions), meaning that the features' instantaneous velocity is $\left(v_{x}=d_{x} / \Delta t, v_{y}=d_{y} / \Delta t\right)$. For the method to work, the time lag must be small enough compared to the lifetime of the features themselves. We isolate in the flow maps the regions of high magnetic activity (active regions, mostly at low to midlatitudes, and diffuse magnetic field, emanating from these regions and dragged toward the poles by the meridional circulation) and their surroundings. We find that the longitudinal averages of the local inflows into active regions have amplitudes comparable to those of the meridional flow and the torsional oscillations at the surface. The latitudinal flow component converges toward the activity belts, while the longitudinal component exhibits retrograde flows present mostly on the poleward side of the active regions.

The active-region flows are converted into north-south helioseismic travel-time shifts by using 3D sensitivity kernels, assuming a radial dependence of the flows. A traveltime sensitivity kernel $\mathcal{K}$ connects a perturbation in the flow field $v$ to the shift in helioseismic travel time $\delta \tau$ induced by this flow (see for instance Fournier et al. (2018) for a computationally-efficient way to compute kernels in the Born approximation). The traveltime perturbation for waves traveling between points $\boldsymbol{r}_{\mathbf{1}}$ and $\boldsymbol{r}_{\mathbf{2}}$ is then the integral over the whole volume of the Sun

$$
\delta \tau\left(\boldsymbol{r}_{\mathbf{1}}, \boldsymbol{r}_{\mathbf{2}}\right)=\int_{\odot} \mathcal{K}\left(\boldsymbol{r}, \boldsymbol{r}_{\mathbf{1}}, \boldsymbol{r}_{\mathbf{2}}\right) \cdot \boldsymbol{v}(\boldsymbol{r}) \mathrm{d} \boldsymbol{r} .
$$

Using 3D kernels is a priori essential because the active-region inflows are not axisymmetric unlike the global meridional circulation. For active-region inflows with a constant depth profile, the longitudinal averages of the forward helioseismic travel-time perturbations computed using the above equation reach up to $\pm 0.3 \mathrm{~s}$ during the peaks of solar activity. When we remove the travel times between points associated with large magnetic field, this amplitude drops to approximately $\pm 0.1 \mathrm{~s}$. For the observed travel times, the amplitude of the meridional flow varies by about $\pm 0.3 \mathrm{~s}$ throughout the solar cycle. Therefore, the active-region flows do not explain in full the solar-cycle variations of the meridional flow. 


\section{Acoustic wave propagation through solar granulation: Validity of effective-medium theories, coda waves $^{1}$}

\section{Abstract}

Context. The frequencies, lifetimes, and eigenfunctions of solar acoustic waves are affected by turbulent convection, which is random in space and in time. Since the correlation time of solar granulation and the periods of acoustic waves ( $\sim 5 \mathrm{~min})$ are similar, the medium in which the waves propagate cannot a priori be assumed to be time independent.

Aims. We compare various effective-medium solutions with numerical solutions in order to identify the approximations that can be used in helioseismology. For the sake of simplicity, the medium is one dimensional.

Methods. We consider the Keller approximation, the second-order Born approximation, and spatial homogenization to obtain theoretical values for the effective wave speed and attenuation (averaged over the realizations of the medium). Numerically, we computed the first and second statistical moments of the wave field over many thousands of realizations of the medium (finite-amplitude sound-speed perturbations are limited to a $30 \mathrm{Mm}$ band and have a zero mean).

Results. The effective wave speed is reduced for both the theories and the simulations. The attenuation of the coherent wave field and the wave speed are best described by the Keller theory. The numerical simulations reveal the presence of coda waves, trailing the ballistic wave packet. These late arrival waves are due to multiple scattering and are easily seen in the second moment of the wave field.

Conclusions. We find that the effective wave speed can be calculated, numerically and theoretically, using a single snapshot of the random medium (frozen medium); however, the attenuation is underestimated in the frozen medium compared to the time-dependent

\footnotetext{
${ }^{1}$ This chapter reproduces the article Acoustic wave propagation through solar granulation: Validity of effective-medium theories, coda waves by P.-L. Poulier, D. Fournier, L. Gizon, and T. L. Duvall Jr., published in Astronomy \& Astrophysics 643, A168 (2020), DOI https://doi.org/10.1051/ 0004-6361/202039201. Reproduced under the terms of the Creative Commons Attribution License (http://creativecommons.org/licenses/by/4.0). Contribution statement: P.-L. P. performed research, accomplished the numerical computations and theoretical derivations, and wrote the paper with contributions from D. F., L. G. and T. L. D. Jr.. D. F. participated in the numerical computations.
} 
2 Acoustic wave propagation through solar granulation: Validity of effective-medium theories, coda waves

medium. Multiple scattering cannot be ignored when modeling acoustic wave propagation through solar granulation.

\subsection{Introduction}

Solar seismic waves interact with small-scale convective motions near the solar surface via a wave scattering process, affecting their properties (e.g., propagation speed, frequency, amplitude, and phase). As the e-folding lifetime of solar granulation is comparable to the period of the waves, the medium may not be assumed to be frozen. Furthermore, the spatial spectrum of convection encompasses all scales, including those that are comparable to the wavelengths of $\mathrm{p}$ and $\mathrm{f}$ modes.

Most approaches that have been proposed so far assume a separation of scales between the waves and the medium. Often the wave period is assumed to be much smaller than the time scale of the evolution of convective flows (Brown 1984, Delache and Fossat 1988, Rosenthal et al. 1999). Murawski and Roberts (1993a,b), using this assumption, derived a model for the scattering of the f mode by granulation using the binary collision approximation (Howe 1971) and found a mode frequency reduction due to the scattering as well as a large attenuation, which compare favorably with observations (Duvall et al. 1998). Other authors assume that the wave period, or the wavelength, is much larger than the temporal, or the spatial, scale of convection, which allows one to apply homogenization techniques (Hanasoge et al. 2013, Bhattacharya et al. 2015).

Numerical simulations provide a useful means to study the interaction of seismic waves with convection (e.g., Ball et al. 2016, Houdek et al. 2017, Schou and Birch 2020). Turbulent convection has an indirect effect on the waves through a change in the average medium (e.g., via a turbulent pressure term) and, in addition, it affects the physics of wave propagation and attenuation via a scattering process.

Here, we study this problem under a highly simplified setup. We consider a onedimensional steady medium that contains sound-speed perturbations over a finite region, but other than that it is uniform. There is no a priori separation of scales in space nor in time between the incoming wave packet and the medium. For relative sound-speed perturbations of a significant amplitude (e.g., 5\% and above) multiple scattering plays a significant role in the redistribution of wave energy. We compare our numerical simulations with theoretical approximations, which are easy to implement in this context.

Since the medium is random in both time and space, we study the effect of the medium on the waves in a statistical sense by computing the first and second moments of the quantities of interest (e.g., the wave field) over many realizations. From the expectation value of the wave field, also known as the coherent or ballistic wave field, we can extract the attenuation and the effective wave speed for example. For the variance of the wave field, we can extract information about the distribution of backward- and forward-scattered energy. This includes late-arrival fluctuations due to multiply-scattered (coda) waves.

We state the problem in Section 2.2 and explain the numerical implementation in Section 2.3. Various effective medium theories are reviewed in Section 2.4. We present our results in Section 2.5, and discuss them in Section 2.6. 
Incoming $\phi_{0}$ Outgoing

Back-scattered

$c_{0}$
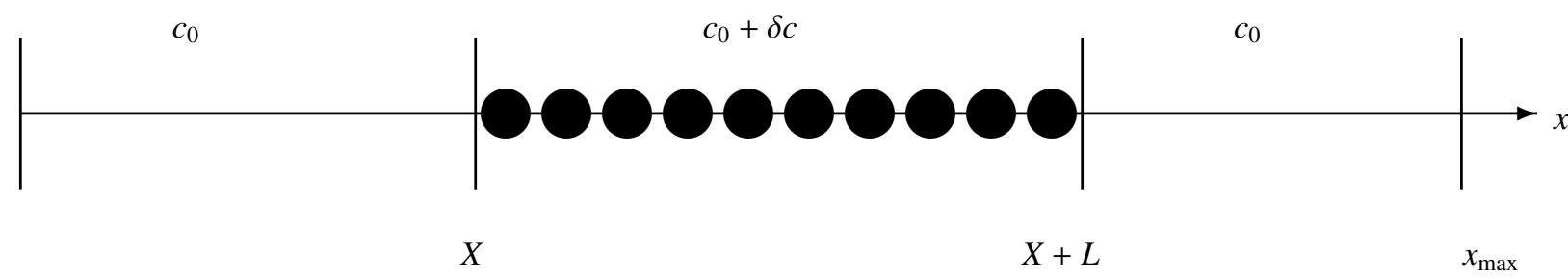

$c_{0}$

Figure 2.1: Schematics of the problem.

\subsection{Statement of the problem}

\subsubsection{The random medium}

We consider a uniform one-dimensional background with sound speed $c_{0}=10 \mathrm{~km} / \mathrm{s}$, a value of the same order of magnitude as the sound speed at the solar surface. We perturb the medium by adding locally a space- and time-dependent random fluctuation:

$$
c(x, t)= \begin{cases}c_{0} & (x<X) \\ c_{0}+\delta c(x, t) & (X \leq x \leq X+L) \\ c_{0} & (x>X+L) .\end{cases}
$$

This is shown in Fig. 2.1, where the filled circles symbolize the fluctuation. In Eq. (2.1), $\delta c$ has a zero mean so that $\langle c\rangle=c_{0}$ where angle brackets denote an expectation value.

The sound-speed perturbation is specified through the autocorrelation

$$
\left\langle\delta c\left(x^{\prime}, t^{\prime}\right) \delta c\left(x^{\prime}+x, t^{\prime}+t\right)\right\rangle=\epsilon^{2} c_{0}^{2} f(x) g(t),
$$

where we assume a separation between time and space. The value of $\epsilon$ is at most 0.1 in our simulations. The random medium can equivalently be characterized by its power spectrum

$$
P(k, \omega)=\int f(x) e^{-i k x} \mathrm{~d} x \int g(t) e^{i \omega t} \mathrm{~d} t=F(k) G(\omega) .
$$

In time, we choose an exponential profile

$$
g(t)=\exp (-|t| / \tau)
$$


2 Acoustic wave propagation through solar granulation: Validity of effective-medium theories, coda waves

where $\tau$ is the e-folding lifetime. For granulation, we have $\tau \approx 400 \mathrm{~s} \mathrm{(e.g.,} \mathrm{Title} \mathrm{et} \mathrm{al.}$ 1989). The temporal power spectrum is Lorentzian,

$$
G(\omega)=\frac{2 \tau}{1+(\omega \tau)^{2}}
$$

In space, we consider two different types of profile. The first choice is an exponential medium (hereafter Medium 1), which will enable us to carry out approximations analytically:

$$
f_{1}(x)=\exp (-|x| / a)
$$

For a granulation-like medium, it is reasonable to choose $a=1 \mathrm{Mm}$. In Fourier space,

$$
F_{1}(k)=\frac{2 a}{1+(k a)^{2}} .
$$

The second choice (hereafter Medium 2) is a spatial power spectrum of the form (e.g., Baran 2013)

$$
F_{2}(k)=C|k|^{\alpha} \exp (-\beta|k|),
$$

where $C=\pi \beta^{\alpha+1} / \Gamma(\alpha+1)$ is a normalization factor such that the spatial autocorrelation function equals 1 at $x=0$. The parameters $\alpha$ and $\beta$ can be tuned to obtain a power spectrum that peaks at the desired wavenumber. Here we fix $\alpha=1$ and $\beta=6.7 \times 10^{-4} R_{\odot}$ where $R_{\odot}=696 \mathrm{Mm}$, such that the spatial power spectrum peaks at $k R_{\odot}=1500$. In real space, for $\alpha=1$, we have

$$
f_{2}(x)=\frac{1-(x / \beta)^{2}}{\left(1+(x / \beta)^{2}\right)^{2}} .
$$

The two power spectra and their corresponding autocorrelation functions are shown in Fig. 2.2.

From the knowledge of the power spectrum $P(k, \omega)$, we can compute a realization of the sound speed perturbations as follows:

$$
\delta c(x, t)=\frac{c_{0}}{(2 \pi)^{2}} \int \sqrt{P(k, \omega)} \mathcal{N}(k, \omega) e^{i(k x-\omega t)} \mathrm{d} \omega \mathrm{d} k,
$$

where $\mathcal{N}(\omega, k)$ is a realization of a complex Gaussian random variable with zero mean and unit variance (the real and the imaginary parts are independent). To ensure that $\delta c(x, t)$ is real, we have $\mathcal{N}(k, \omega)=\mathcal{N}^{*}(-k,-\omega)$. This way to proceed is based on the assumptions of stationarity and horizontal spatial homogeneity of the medium (e.g., Gizon and Birch 2004).

\subsubsection{The wave equation}

The displacement $\boldsymbol{\xi}$ of acoustic waves is given by (Lynden-Bell and Ostriker 1967)

$$
\partial_{t}^{2} \boldsymbol{\xi}-\frac{1}{\rho} \nabla\left(\rho c^{2} \nabla \cdot \boldsymbol{\xi}\right)=0
$$

Here, we have ignored gravity, rotation, damping as well as any background flows. This equation has been derived in a background medium where the parameters $\rho$ and $c$ are 

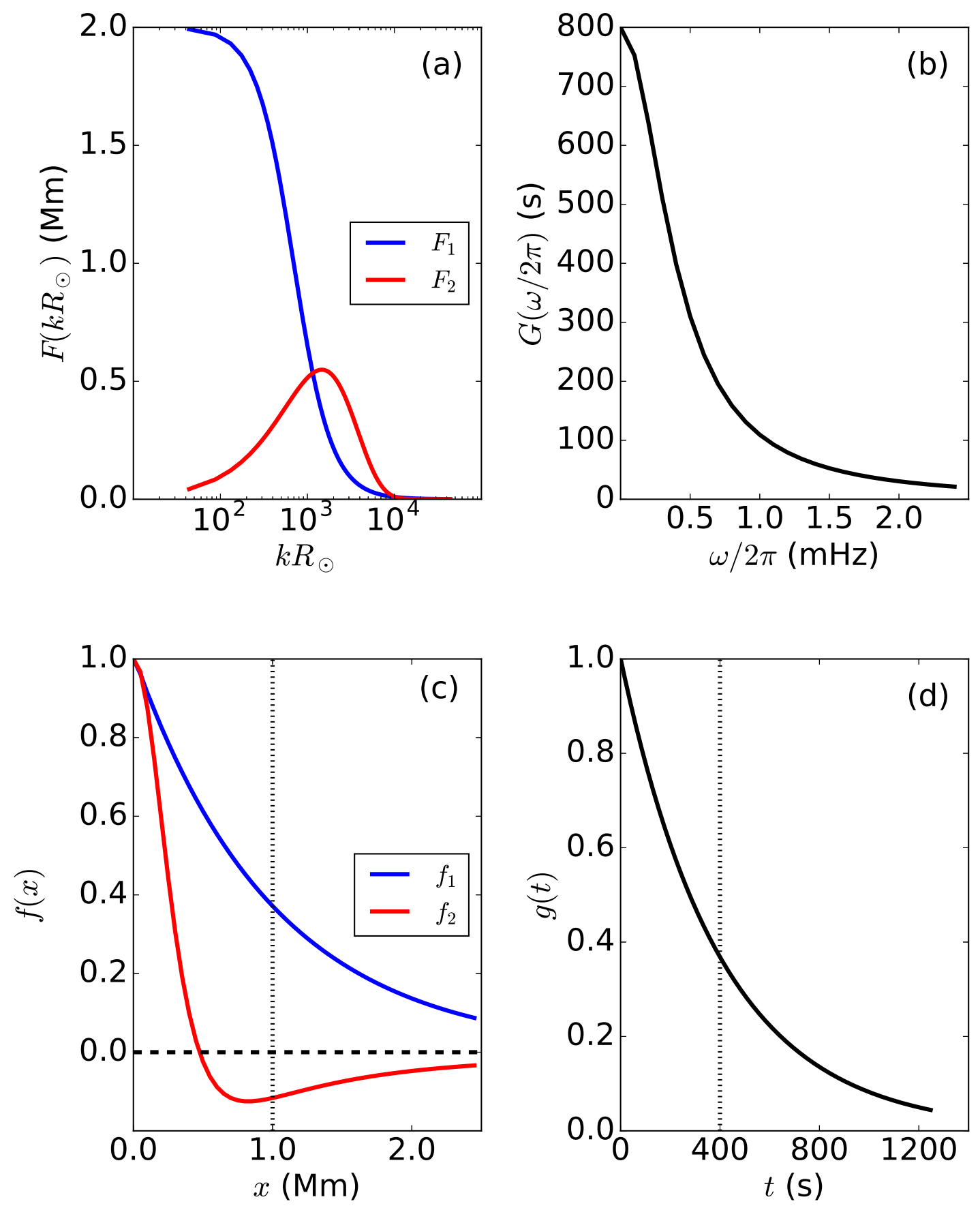

Figure 2.2: Panel $a$ : Power spectrum as a function of the adimensional wave number $k R_{\odot}$. Panel b: Power spectrum as a function of frequency. Panel $c$ : Spatial autocorrelation. Panel d: Temporal autocorrelation. The vertical dotted lines are drawn at the values of the correlation parameters chosen for medium 1, namely $\tau=400 \mathrm{~s}$ and $a=1 \mathrm{Mm}$.

independent of time. However, Legendre (2003) showed that this formulation remains valid for a time-varying medium. Taking the divergence of Eq. (2.11) and denoting $\phi=$ 
2 Acoustic wave propagation through solar granulation: Validity of effective-medium theories, coda waves

$\nabla \cdot \boldsymbol{\xi}$, we obtain

$$
\partial_{t}^{2} \phi-\nabla \cdot\left(\frac{1}{\rho} \nabla\left(\rho c^{2} \phi\right)\right)=0 .
$$

In this paper, we assume that the density is constant and consider the following 1D acoustic wave equation

$$
\partial_{t}^{2} \phi-\partial_{x}^{2}\left(c^{2} \phi\right)=0 .
$$

We implement two numerical codes. The first code is a time-domain code to study the propagation of a wave packet through a time-dependent random medium, based on Eq. (2.13). As initial condition, we inject at location $x_{0}<X$ a wave packet of central frequency $\omega_{0}$ and frequency width $\sigma$ :

$$
\phi(x, 0)=\phi_{0}(x, 0), \quad \partial_{t} \phi(x, 0)=\partial_{t} \phi_{0}(x, 0),
$$

where

$$
\phi_{0}(x, t)=\exp \left[-\frac{\sigma^{2}}{2}\left(\frac{x-x_{0}}{c_{0}}-t\right)^{2}\right] \cos \left[\omega_{0}\left(\frac{x-x_{0}}{c_{0}}-t\right)\right] .
$$

As shown in the schematics of Fig. 2.1, the incoming wave packet first travels in the $+x$ direction in the homogeneous medium, experiences scattering inside the perturbed medium, then comes out (outgoing wave packet) and propagates in the $+x$ direction in the homogeneous medium. Part of the wave packet is back-scattered and travels in the $-x$ direction. The simulation box is large enough so that the wave packet is not affected by the computational boundaries at $x=0$ and $x=x_{\max }$.

The second code is a frequency-domain code to study the wave field in a frozen medium $(\tau \rightarrow \infty)$. For a sound speed that does not depend on time, we can take the temporal Fourier transform of Eq. (2.13) to obtain the wave equation in the frequency domain, i.e. the Helmholtz equation

$$
\partial_{x}^{2}\left(c^{2} \tilde{\phi}(x, \omega)\right)+\omega^{2} \tilde{\phi}(x, \omega)=0,
$$

with Dirichlet boundary condition at $x=0$,

$$
\tilde{\phi}(0, \omega)=1,
$$

and the Sommerfeld outgoing radiation condition

$$
\partial_{x} \tilde{\phi}\left(x_{\max }, \omega\right)=\frac{i \omega}{c\left(x_{\max }\right)} \tilde{\phi}\left(x_{\max }, \omega\right) .
$$

The tilde denotes the temporal Fourier transform.

\subsubsection{Characterizing the wave field}

The wave field is affected randomly by the perturbations. The statistical effects can however be studied by looking at the moments of the wave field, i.e. by doing some averages over the realizations of the random medium.

In particular, the coherent wave field is attenuated because each wave packet travels in a different random realization of the medium and is deformed in a different way. 
This damping is related to the lifetime of the average acoustic wave. The coherent wave field also propagates with a different velocity than $c_{0}$, depending on frequency, called the effective wave speed.

An approximate representation of the coherent wave field inside the perturbed medium is therefore

$$
\langle\phi\rangle \sim e^{i k(\omega) x-i \omega t}=e^{-k_{i}(\omega) x} e^{i k_{r}(\omega) x-i \omega t},
$$

where the effective wave number is

$$
k_{r}(\omega)=\operatorname{Re} k(\omega),
$$

and the spatial attenuation is

$$
k_{i}(\omega)=\operatorname{Im} k(\omega) .
$$

The effective wave speed is defined by

$$
c_{\mathrm{eff}}(\omega)=\frac{\omega}{k_{r}(\omega)}
$$

Similarly, we define $k_{0}(\omega)$, the wave number for an unperturbed wave field, such that

$$
c_{0}=\frac{\omega}{k_{0}(\omega)} .
$$

We want to solve the (simplified) problem of acoustic wave scattering numerically and find which approximations work to retrieve the coherent wave field. In particular, we check whether we can get rid of the time dependence and assume a frozen medium.

Furthermore, we want to investigate the phenomenon of multiple scattering due to the finite-amplitude perturbations. This is more easily done by looking at the second moment (variance) of the wave field. It contains information that is otherwise zeroed out by doing a mere average. By doing so, the coda waves, which trail the ballistic wave packet and are often studied in seismology, can be readily observed.

\subsection{Numerical methods}

\subsubsection{Numerical scheme to solve for $\phi(x, t)$}

In order to solve numerically Eq. (2.13), we use an explicit finite-difference scheme of second order. We choose $\omega_{0} / 2 \pi=3 \mathrm{mHz}$ and $\sigma / 2 \pi=1 \mathrm{mHz}$ so that the frequency range of study is 1 to $5 \mathrm{mHz}$, which is a reasonable choice for solar acoustic waves. The wave packet is initially at $x_{0}=100 \mathrm{Mm}$, while $x_{\max }=200 \mathrm{Mm}$ and $t_{\max }=10000 \mathrm{~s}$. We set $X=120 \mathrm{Mm}$ and $L=30 \mathrm{Mm}$. The resolutions for the simulations are $\Delta x=50 \mathrm{~km}$ and $\Delta t=2.5 \mathrm{~s}$, so that $c_{0} \Delta t / \Delta x=0.5<1$.

An example of time-domain simulation with medium 1 is shown in the online movie and in Fig. 2.3. The wave packet begins to be perturbed when it enters the random medium. Most of the signal is transmitted forward roughly in the form of a wave packet (ballistic wave packet). Small oscillations trail that signal, propagating either forward or backward. Once out of the perturbation, the shape of the wave packet is not modified anymore. 
2 Acoustic wave propagation through solar granulation: Validity of effective-medium theories, coda waves
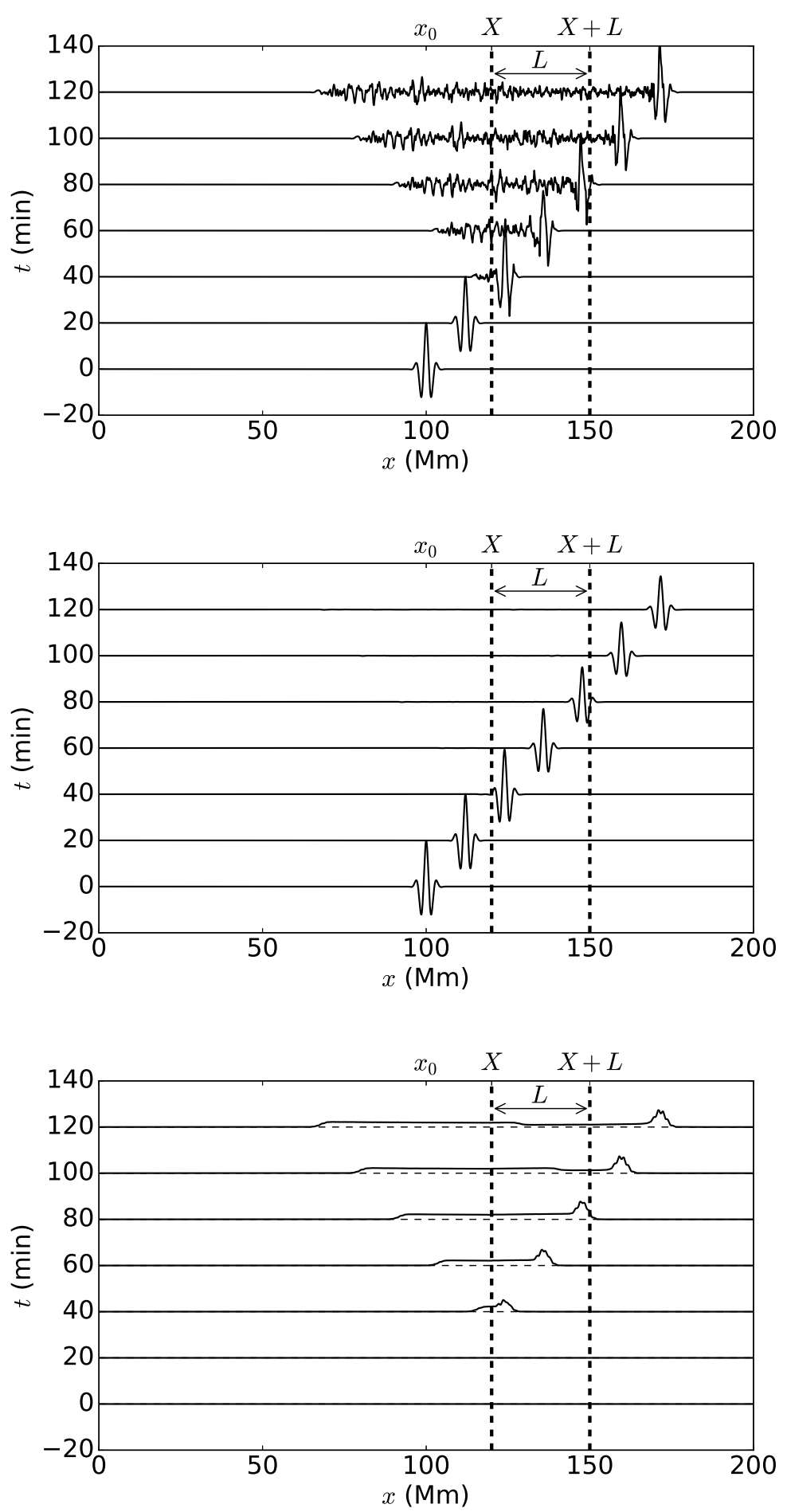

Figure 2.3: Top: Wave packet propagation through a realization of a random medium (medium 2, with $\epsilon=0.1$ and $\tau=400 \mathrm{~s}$ ) located between the vertical dashed lines at different time steps. Middle: Average over 10000 realizations. Bottom: Square root of the variance of the wave field. See the movie online. 


\subsubsection{Numerical scheme to solve for $\tilde{\phi}(x, \omega)$}

The code uses a second-order discretization scheme with a spatial resolution $\delta x=4 \mathrm{~km}$. A tridiagonal system is inverted with the tridiagonal matrix algorithm (Thomas algorithm). $c_{0}, x_{\max }, X$ and $L$ are the same as for the time-domain code. The resolution is done for frequencies between 1 and $5 \mathrm{mHz}$.

\subsubsection{Measuring the attenuation}

Following Aki and Richards (2002), after propagating between two points $x_{1}$ and $x_{2}\left(x_{2}>\right.$ $x_{1}$ ) in an attenuating medium, a plane wave is damped by a factor

$$
e^{-k_{i}\left(x_{2}-x_{1}\right)}
$$

The spatial attenuation $k_{i}$ could be measured from the amplitude difference between the incoming and the outgoing wave packets. However, this method leads to artifacts due to boundary effects occurring at the edges of the random medium. Therefore, we rather consider the wave packet inside the perturbation. We take $x_{1}=126 \mathrm{Mm}$ and $x_{2}=144 \mathrm{Mm}$, each point being $6 \mathrm{Mm}$ away from the edge of the perturbation. As shown in Fig. 2.4, we take the temporal Fourier transform of $\langle\phi(x, t)\rangle$ where $x \in\left[x_{1}, x_{2}\right]$. The power of the signal has been attenuated during the propagation from $x_{1}$ to $x_{2}$. At each frequency, we then fit a first order polynomial to the natural logarithm of the norm of the Fourier component in order to retrieve the decay coefficient.

\subsubsection{Measuring the effective wave speed}

We first apply a temporal Fourier transform to $\langle\phi\rangle$. Then we fit to $\operatorname{Re}(\langle\tilde{\phi}(x, \omega)\rangle)$ (we could have chosen the imaginary part arbitrarily), in the perturbed region, an exponentially decreasing oscillatory function where the decay rate has been determined via the method to measure the attenuation from the previous section. More precisely, we fit

$$
A e^{-k_{i}(x-X)} \cos \left(\frac{\omega}{c_{\mathrm{eff}}(\omega)}\left(x-x_{s}\right)\right)
$$

where $A, x_{s}$, and $c_{\text {eff }}$ are the free parameters, with $x_{s}$ being a phase shift. A similar fit is done on $\phi_{0}$ to take numerical dispersion into account. This is illustrated in Fig. 2.5.

\subsection{Effective medium theories}

Depending on the values of the parameters, in particular $k_{0} a$ and $\epsilon$, different theories can be used to compute the effective parameters $c_{\text {eff }}$ and $k_{i}$. For a medium whose spatial scale is much less than the wavelength $(\lambda \gg a$, regime of Rayleigh scattering), the homogenization method is appropriate and gives an effective sound speed $c_{\text {eff }}=c_{0}\left(1-3 / 2 \epsilon^{2}\right)$ (derivation for a frozen medium in Appendix 2.7.4). On the other hand, for small wavelengths ( $\lambda \ll a$, small-angle scattering regime), the geometrical optics approach is relevant and implies $c_{\text {eff }}=c_{\text {ray }}=c_{0}\left(1-\epsilon^{2}\right)$ (derivation for a frozen medium in Appendix 2.7.5). These two approaches give an effective wave speed that is independent of frequency and of the 
2 Acoustic wave propagation through solar granulation: Validity of effective-medium theories, coda waves
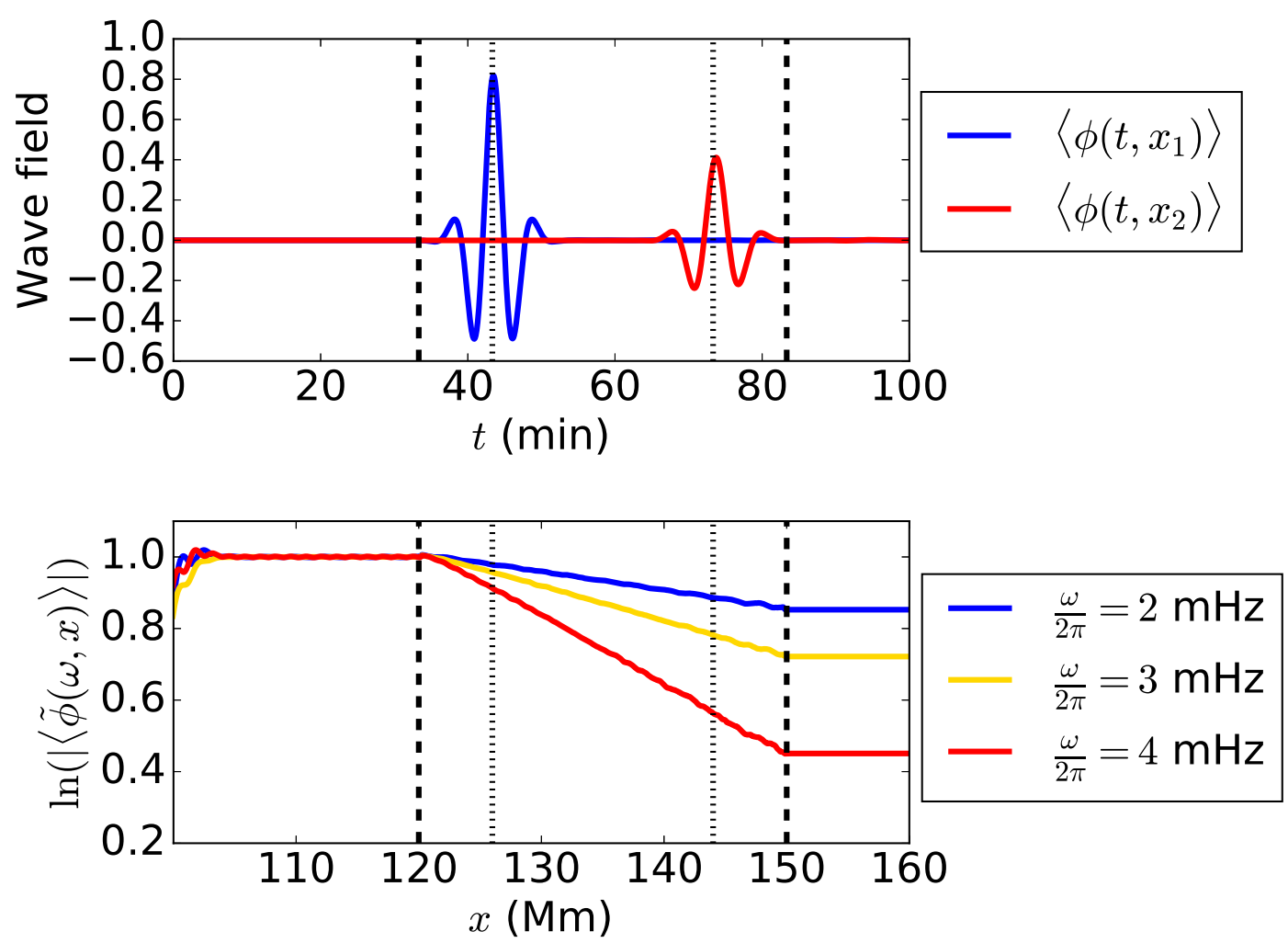

Figure 2.4: Measuring the attenuation with the temporal code. Top: Coherent wave packet at $x_{1}=126 \mathrm{Mm}$ (blue) and $x_{2}=144 \mathrm{Mm}$ (red). Bottom: Natural log of the spectrum of the coherent wave packet at different frequencies. We fit its slope between the vertical dotted lines, corresponding to $\left[x_{1}, x_{2}\right]$. The vertical dashed lines delimit the location of the perturbation in time (for a wave packet propagating at $c_{0}$ ) and in space. In the figure, the Fourier components have been normalized so that they have the same amplitude before entering the perturbation.

power spectrum of the perturbation but do not provide any attenuation. Another caveat is that in our model, we are in the regime of Mie scattering or large-angle scattering (Aki and $\mathrm{Wu} 1988$ ) because $\lambda \simeq 3.3 a$. The wave number can therefore not be considered large nor small compared to 1 and other theories may be required.

We explore two other derivations in the case of small perturbations $(\epsilon \ll 1)$. In this regime, two methods are used: the Keller solution, derived for a frozen (Appendix 2.7.1) or time-dependent medium (Appendix 2.7.2); the Born second-order approximation for a frozen medium (derived in Appendix 2.7.3). The Keller and the Born solutions converge toward the same values for small perturbations $(\epsilon=0.01$ for instance). However, at $\epsilon=0.1$, the Born solution is very different from the Keller one, and it is not possible to fit a function of the form of Eq.(2.19). We come back to this point in Section 2.5.1.

If $k_{0} a \ll 1$ or $k_{0} a \gg 1$, the effective wave speed obtained from the Keller theory converges toward the results of the homogenization and geometrical optics approaches, respectively. Table 2.1 summarizes the expressions for a frozen medium 1 (for which the analytical expressions can be easily derived) as $\epsilon \rightarrow 0$. We check the agreement of these 

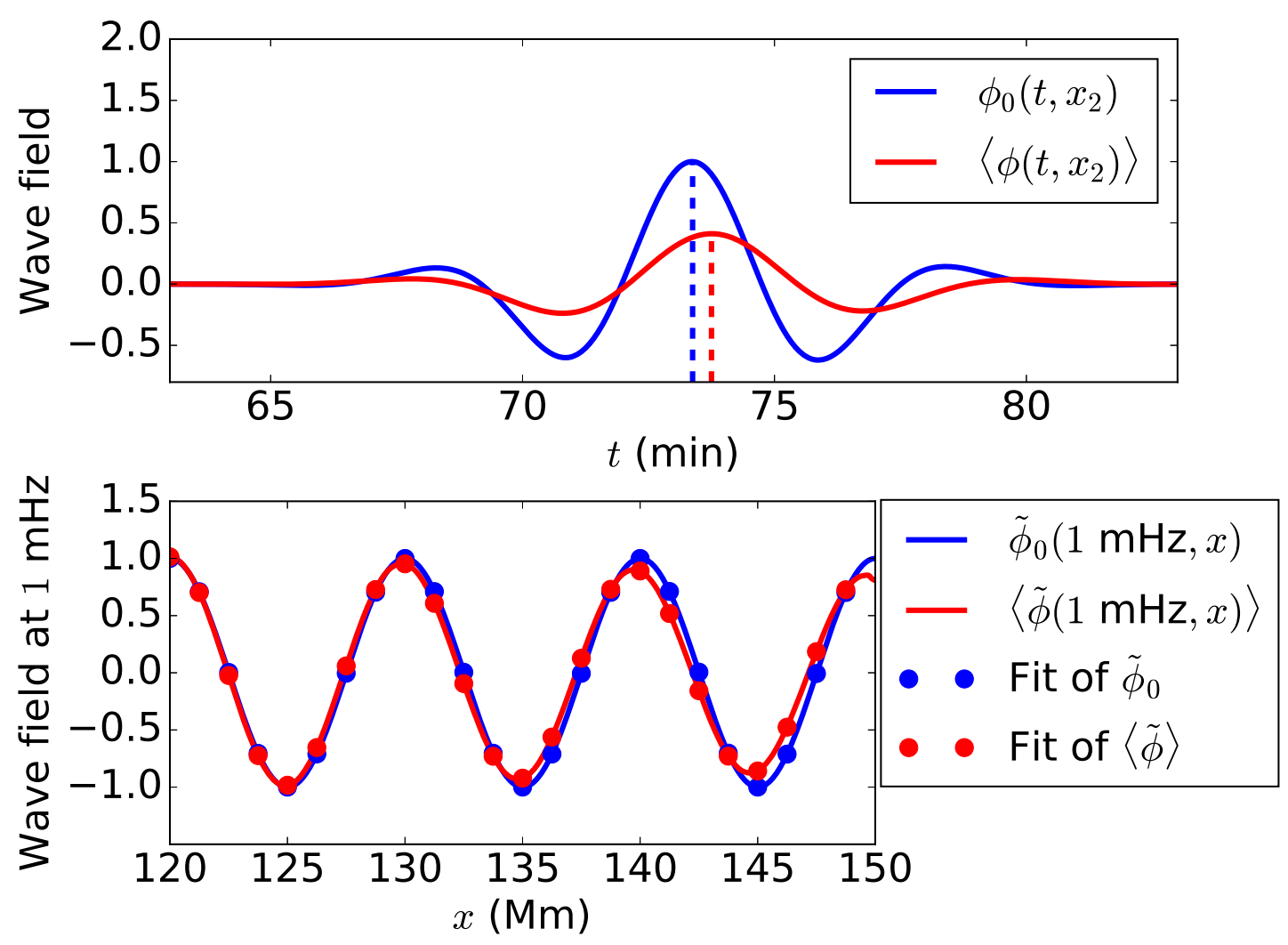

Figure 2.5: Measuring the effective wave speed. Top: Coherent wave packet (red curve) experiencing a travel time shift compared to the unperturbed wave packet (blue curve). The vertical dashed lines represent the arrival times at $x_{2}$. Bottom: Fit of a decaying cosine to the real part of the temporal Fourier transform in the random region, at $\omega / 2 \pi=1 \mathrm{mHz}$.

theories with our simulations in Appendix 2.7.6. The Keller and the Born second-order theories are consistent and predict that $k_{i}$ is proportional to $k_{0}^{2}$ while the effective wave speed difference is essentially independent of $k_{0}$ provided that $k_{0} a \gtrsim 1$. For comparison purposes, we note that Bourret (1963) and Sato et al. (2012) (pp. 214-220) found that for a 3D frozen medium with an exponential autocorrelation (i.e., similar to medium 1 ), the attenuation is proportional to $k_{0}^{4}$ for $k_{0} a \ll 1$ and to $k_{0}^{2}$ for $k_{0} a \gg 1$ while the behavior of the effective wave speed is in qualitative agreement. In particular, the effective wave speed is always less than $c_{0}$. On the other hand, van der Baan (2001) used the wave localization theory to make use of so-called self-averaging quantities; he derived the effective medium using one realization of a one-dimensional perturbation in density and bulk modulus. He found that the attenuation coefficient tends toward a constant value at high $k_{0} a$, while the effective wave speed difference is positive, in agreement with previous studies (e.g., Müller et al. 1992). The sign of the difference $c_{\text {eff }}-c_{0}$ and the dependence of the attenuation on frequency therefore seem to depend strongly on the equation that is solved. 
2 Acoustic wave propagation through solar granulation: Validity of effective-medium theories, coda waves

Table 2.1: Theories used in this paper for the effective wave speed and attenuation in a frozen medium as $\epsilon \rightarrow 0$, and their range of validity.

\begin{tabular}{cccc}
\hline \hline Theory & Validity range & $k_{i}$ & $c_{\text {eff }}$ \\
\hline Keller 1964 & $\epsilon \ll 1$ & $\epsilon^{2} k_{0}\left(k_{0} a+\frac{k_{0} a}{1+4\left(k_{0} a\right)^{2}}\right)$ & $c_{0}\left(1-\frac{\epsilon^{2}}{2}\left[3-\frac{4\left(k_{0} a\right)^{2}}{1+4\left(k_{0} a\right)^{2}}\right]\right)$ \\
Born (2nd order) & $\epsilon^{2}\left(k_{0} a\right)^{2} \frac{L}{a} \ll 1^{(\dagger)}$ & $\simeq^{(\ddagger)} \epsilon^{2} k_{0}\left(k_{0} a+\frac{k_{0} a}{1+4\left(k_{0} a\right)^{2}}\right)$ & $\simeq^{(\ddagger)} c_{0}\left(1-\frac{\epsilon^{2}}{2}\left[3-\frac{4\left(k_{0} a\right)^{2}}{1+4\left(k_{0} a\right)^{2}}\right]\right)$ \\
Homogenization & $k_{0} a \ll 1$ & Not applicable & $c_{\mathrm{h}}=\left\langle c^{-2}\right\rangle^{-1 / 2} \simeq c_{0}\left(1-\frac{3}{2} \epsilon^{2}\right)$ \\
Geometrical optics & $k_{0} a \gg 1, k_{0} a \gg 2 \pi \frac{L}{a}, \epsilon \ll 1^{(\#)}$ & Not applicable & $c_{\text {ray }}=\left\langle c^{-1}\right\rangle^{-1} \simeq c_{0}\left(1-\epsilon^{2}\right)$ \\
\hline
\end{tabular}

The Keller and the Born theories are for medium 1. (†) Approximation for $k_{0} a>1$ of Rytov et al. (1989b) who made the derivation for a Gaussian autocorrelation function and single scattering. ${ }^{(\ddagger)}$ The dominant term is that of Keller for small perturbations (see for example Fig. 2.12). " See Rytov et al. (1989a).

\subsection{Results and comparisons}

We compute the properties of the effective medium using the procedure explained in Sections 2.3.3 and 2.3.4, with $\epsilon=0.1$ and $a=1 \mathrm{Mm}$. The 1- $\sigma$ error bars shown later on the attenuation and wave speed measurements are obtained from ten sets of $10^{4}$ realizations.

\subsubsection{Coherent wave field}

We first reconstruct the theoretical coherent wave field obtained when using the various theories. For all of them (except the Born theory that provides directly the wave field), we assume the form written in Eq. (2.19). In Fig. 2.6, we plot $\operatorname{Re}(\langle\tilde{\phi}(3 \mathrm{mHz}, x)\rangle)$ $(x \in[X, X+L])$ for a frozen medium 1. Clearly the Keller approximation does the best job at approximating the true (numerical) solution. As mentioned before, neither the homogenization technique nor the geometrical optics makes an attenuation emerge. The Born solution is a good approximation on about half of the random medium at this frequency, before it becomes out of phase with the numerical solution while its amplitude also starts to increase. The discrepancy is worse and arises earlier in the medium for higher frequencies. Thus the Born approximation, although similar to the Keller approximation when $\epsilon \rightarrow 0$, performs poorly for a $10 \%$ perturbation in a medium of size $L=30 \mathrm{Mm}$.

\subsubsection{Attenuation}

Fig. 2.7 shows the measured attenuation for simulations with $\tau=400 \mathrm{~s}$ and $\tau \rightarrow \infty$. The case $\tau=1$ day (not shown on the plot) lies within the error bars of the curve for the frequency code, which is to be expected as the typical time scales involved (the period of the wave, about 5 minutes, and the time it takes for it to travel through the medium, about $1 \mathrm{~h}$ ) are much less than one day. We superimpose the attenuation that one expects from the time-dependent Keller theory.

The attenuation by medium 1 is an increasing function of frequency, with a value of about $1.5 \%$ of the wave number at $3 \mathrm{mHz}$ for $\tau=400 \mathrm{~s}$. The ratio $k_{i} / k_{0}$ is a linear function of frequency, meaning that $k_{i}$ is quadratic, as expected from the Keller theory. For medium 2 however, the attenuation is not quadratic. It reaches about $0.5 \%$ of the 


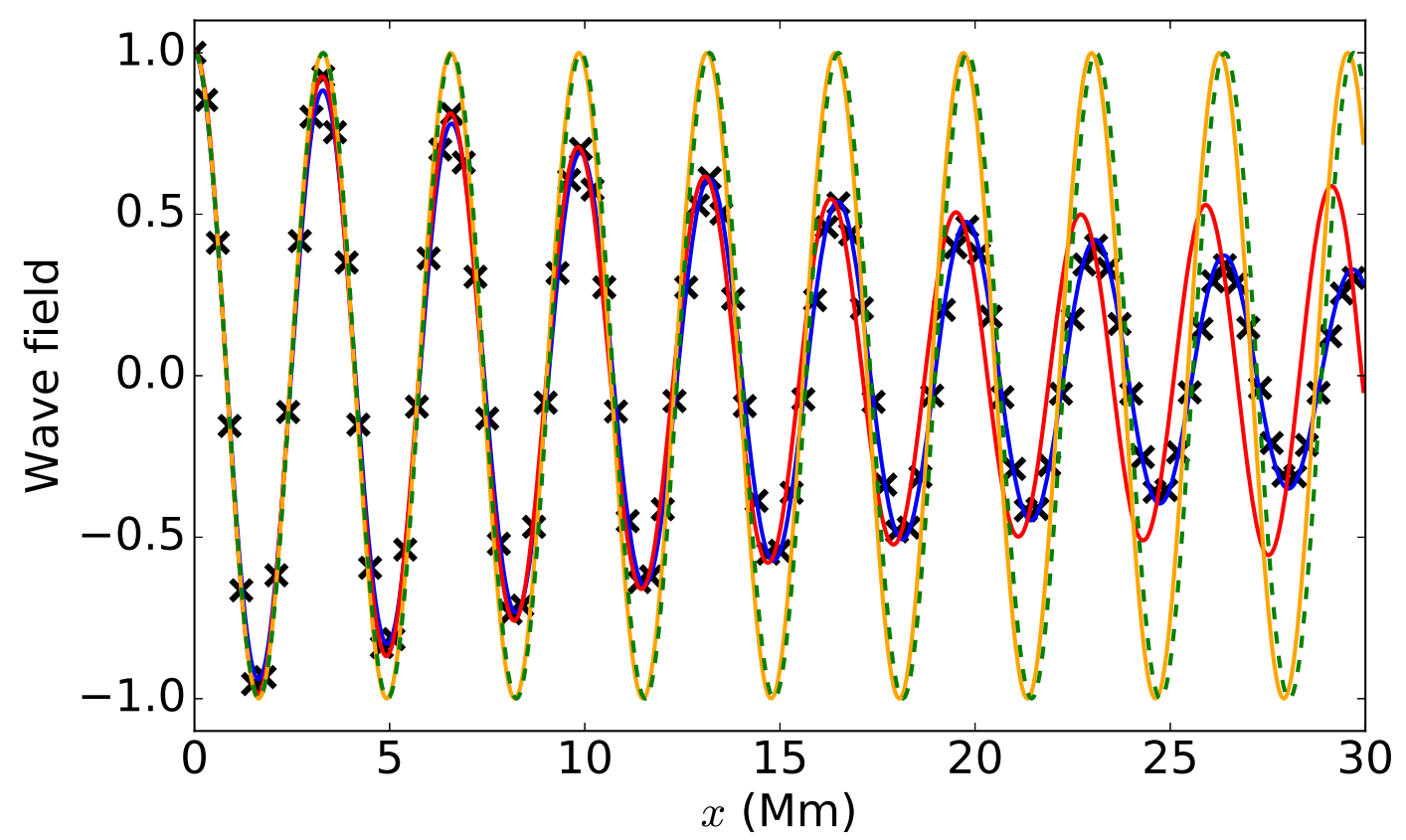

Figure 2.6: Theoretical solutions compared with the coherent wave field inside the random medium from the numerical simulation, for a frozen medium 1, at $3 \mathrm{mHz}$. Keller: blue. Born: red. Homogenization: orange. Geometrical optics: dashed green. The black crosses are the numerical solution.

wave number at $3 \mathrm{mHz}$, which is smaller than the medium 1 value by a factor 3 . The smaller attenuation values are caused by the lack of power toward low wave numbers in the spectrum of the perturbation: the absence of large scales in the perturbation means that the incoherence between the realizations of the wave packets occurs preferentially on small scales, thereby decreasing the overall broadening of the wave packet. In medium 2 , the ratio $k_{i} / k_{0}$ stabilizes above $5 \mathrm{mHz}$ for $\tau=400 \mathrm{~s}$, while it reaches a maximum at about $3 \mathrm{mHz}$ for $\tau \rightarrow \infty$. This may indicate that there is a preferred scale of damping of the coherent wave field.

\subsubsection{Effective wave speed}

Fig. 2.8 shows the effective wave speed computed with for $\tau=400 \mathrm{~s}$ and $\tau \rightarrow \infty$, as well as the time-dependent Keller theory, the (frozen) spatial homogenization solution and the (frozen) geometrical optics solution. Like for the attenuation, the case $\tau=1$ day (not shown) lies within the error bars of the curve for the frequency code. The effective wave speed is less than the unperturbed sound speed $c_{0}$. This is due in part to waves being scattered back and forth, contributing to the overall transmitted signal but at a later time than the unperturbed wave. The second reason is the delay experienced by forwardscattered waves. Indeed, in the regime of geometrical optics $(\lambda / a \ll 1)$ where scattering occurs essentially forward, the effective wave speed is given by the geometric velocity $c_{\text {ray }}=\left\langle c^{-1}\right\rangle^{-1}<c_{0}$. 
2 Acoustic wave propagation through solar granulation: Validity of effective-medium theories, coda waves

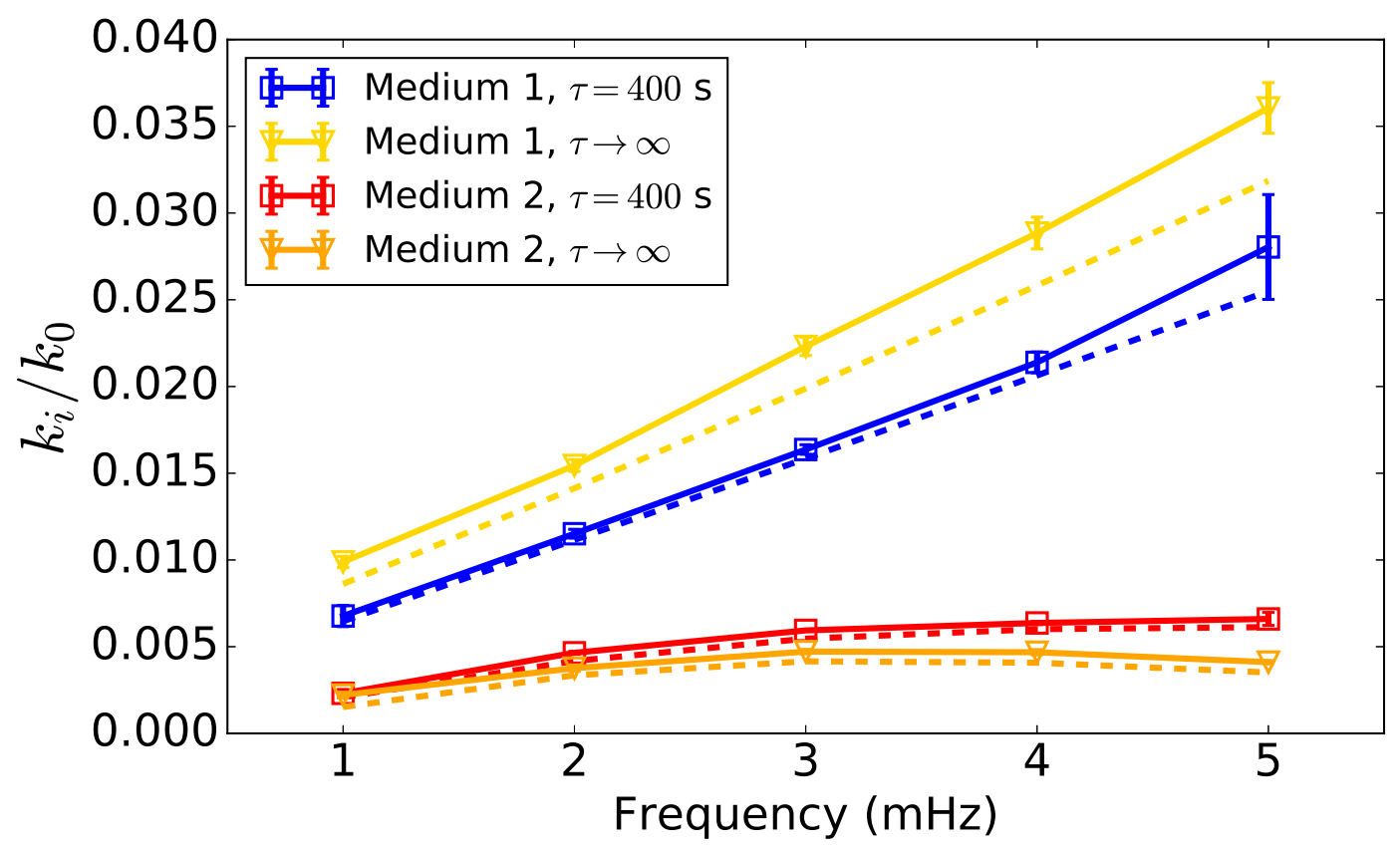

Figure 2.7: Attenuation of the coherent wave packet vs frequency for media 1 and 2, after propagation through a band of perturbed medium. The 1D theory from Keller is overplotted in dashed lines. 1- $\sigma$ error bars are shown.

The effective wave speed in medium 1 is an increasing function of frequency, with a shift from $c_{0}$ by about $-0.7 \%$ at $3 \mathrm{mHz}$ for $\tau=400 \mathrm{~s}$. The Keller theory is in relative agreement for low frequencies $(f \leq 2 \mathrm{mHz})$ but it predicts a constant wave speed at higher frequencies. On the other hand, the measured effective wave speed in medium 2 clearly changes from the homogenized velocity $c_{\mathrm{h}}$ at $1 \mathrm{mHz}$ to the geometric velocity $c_{\text {ray }}$ at $5 \mathrm{mHz}$. We note a remarkable agreement at all frequencies between the simulations and the Keller theory for medium 2.

\subsubsection{Variance of wave field}

The mean of the perturbation is zero, therefore looking at the coherent wave field may not be enough to directly detect multiple scattering because one would only see oscillations mixed within the noise. In the regime of strong perturbations, the coherent part would vanish and only the fluctuating part would remain, solely accessible via second order moments. One can for instance look at the envelope of the signal by studying the variance of the wave field.

As shown in Fig. 2.9 and in the online movie, it is composed of three parts: a peak corresponding to the variance of the ballistic wave packet, coda waves (late-arriving waves) propagating forward, and coda waves propagating backward. The forward-propagating coda results from waves back-scattered an even number of times in the perturbed medium. The backward-propagating coda forms a plateau of width $2 L$ and results from single backscattering. In geophysics, a connection has been made between the functional form of the 


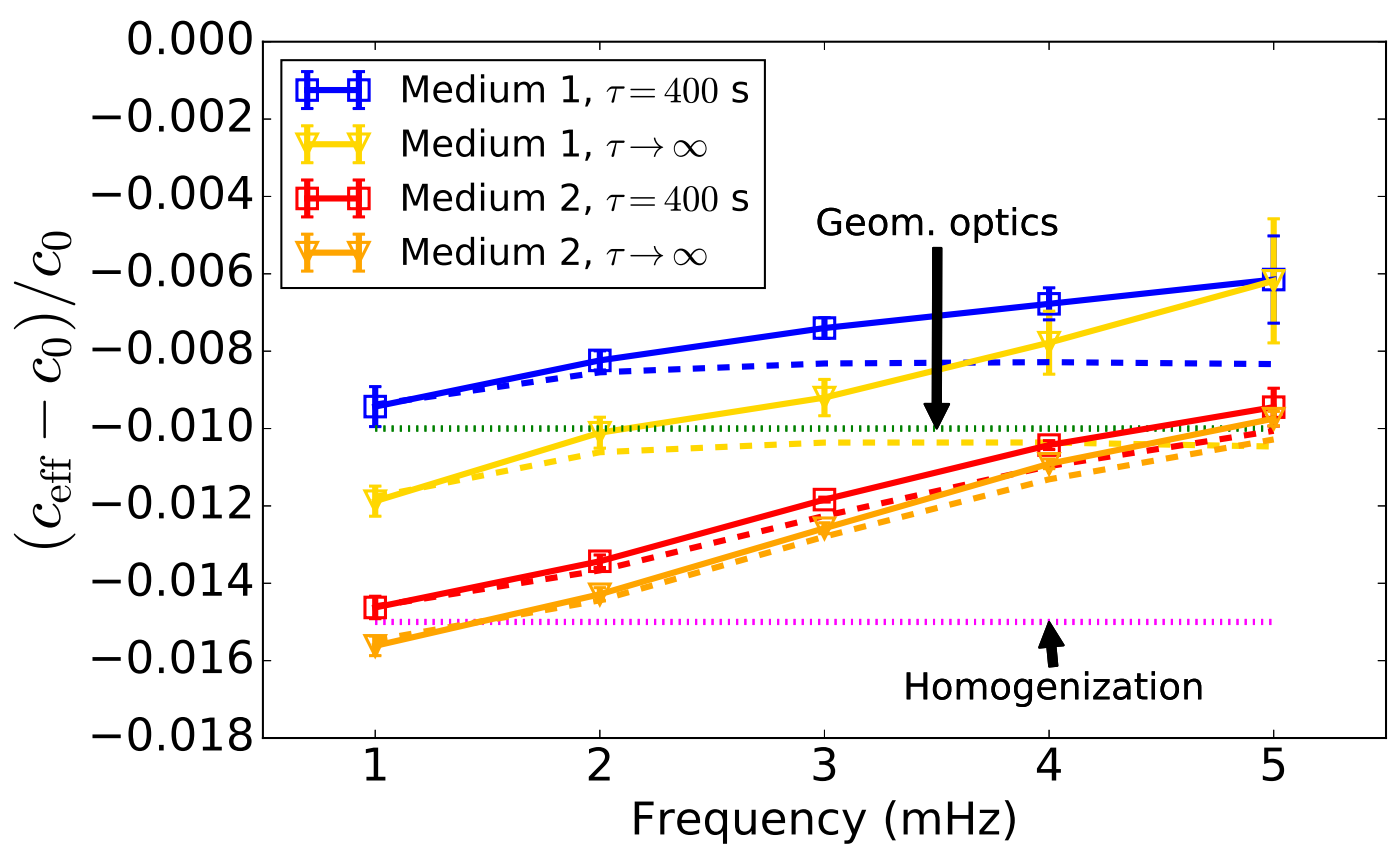

Figure 2.8: Speed of the coherent wave packet vs frequency for media 1 and 2, after propagation through a perturbed medium. The 1D theory adapted from Keller (dashed lines) as well as the prediction from the homogenization theory and the geometrical optics are shown. 1- $\sigma$ error bars are shown.

coda in time domain and the complexity of the scattering medium (e.g., Sato et al. 2012).

We decompose the domain in three regions (before, after and in the random medium) and integrate spatially the variance over each of these three regions at $t_{m}=8500$ s, i.e. after the coherent wave packet went through the random medium and just after the plateau of back-scattered signal went out of it:

$$
\begin{aligned}
& \mathcal{E}_{b s c}=\int_{0}^{X} \operatorname{Var}\left(\phi\left(x, t_{m}\right)\right) \mathrm{d} x, \\
& \mathcal{E}_{\text {out }}=\int_{X+L}^{x_{\max }} \operatorname{Var}\left(\phi\left(x, t_{m}\right)\right) \mathrm{d} x, \\
& \mathcal{E}_{t r}=\int_{X}^{X+L} \operatorname{Var}\left(\phi\left(x, t_{m}\right)\right) \mathrm{d} x .
\end{aligned}
$$

It gives us a measurement of the variance that, respectively, has been back-scattered, transmitted or is still trapped in the slab at this particular time. For medium 2, the backscattered variance makes up for about $50 \%$ of the total variance for $\tau=400 \mathrm{~s}$, and $75 \%$ for $\tau=1$ day. The reason for these high amounts is that the spectrum of medium 2 peaks at small scales, therefore more back-scattering takes place than for instance in medium 1 where these values become respectively $20 \%$ and $15 \%$. 
2 Acoustic wave propagation through solar granulation: Validity of effective-medium theories, coda waves
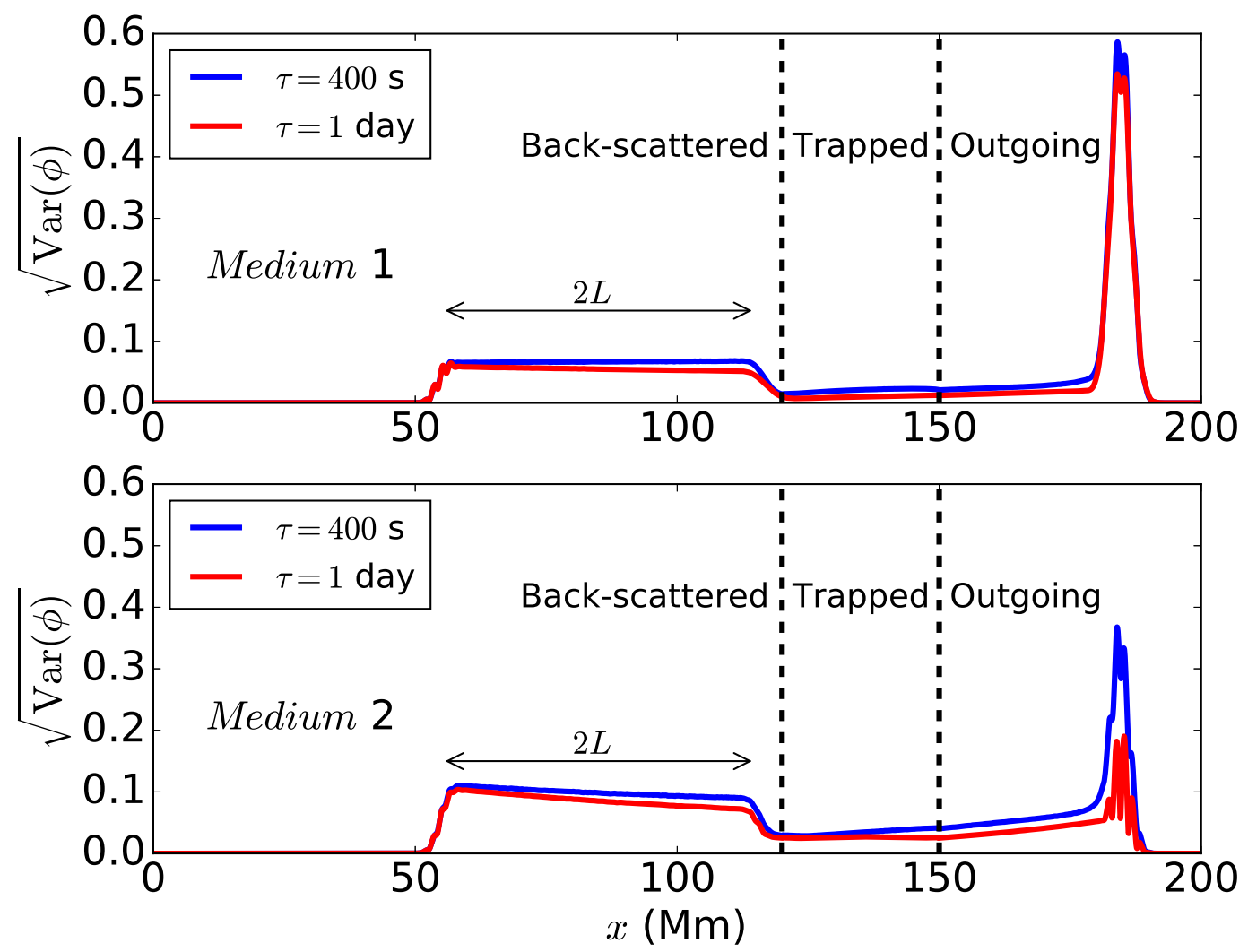

Figure 2.9: Square root of the variance of the wave field as a function of position at a given time $t=8500 \mathrm{~s}$. Top: Medium 1. Bottom: Medium 2. See the movie online.

\subsubsection{Dependence on correlation time of the medium}

Calculations of an effective medium are easier to carry when the perturbation is frozen because one can work directly in the frequency domain. Therefore, we study here how the effective parameters $k_{i}$ and $c_{\text {eff }}$ depend on the correlation time of the medium.

Fig. 2.10 shows the relative errors in the attenuation, $e_{k_{i}}$, and in the effective wave speed difference, $e_{c}$, between a given correlation time and the $\tau \rightarrow \infty$ case at 2, 3 and $4 \mathrm{mHz}$, for medium 2:

$$
\begin{aligned}
e_{k_{i}}(\omega, \tau) & =\frac{k_{i}(\omega, \tau)-k_{i}(\omega, \infty)}{k_{i}(\omega, \infty)}, \\
e_{c}(\omega, \tau) & =\left(\frac{c_{\mathrm{eff}}(\omega, \tau)-c_{0}}{c_{0}}-\frac{c_{\mathrm{eff}}(\omega, \infty)-c_{0}}{c_{0}}\right)\left(\frac{c_{\mathrm{eff}}(\omega, \infty)-c_{0}}{c_{0}}\right)^{-1} \\
& =\frac{c_{\mathrm{eff}}(\omega, \tau)-c_{\mathrm{eff}}(\omega, \infty)}{c_{\mathrm{eff}}(\omega, \infty)-c_{0}} .
\end{aligned}
$$

$e_{k_{i}}$ being generally positive, the attenuation is underestimated by the frozen-medium approximation. Our understanding is that since the power of the perturbation mostly lies at high wave numbers, the attenuation mostly comes from the small-scale incoherence between the realizations of the wave packets. Therefore, there must be two regimes: one at small values of $\tau$ where the attenuation increases with $\tau$, and one at greater values of $\tau$ where the attenuation decreases, because persisting scatterers start to create less 

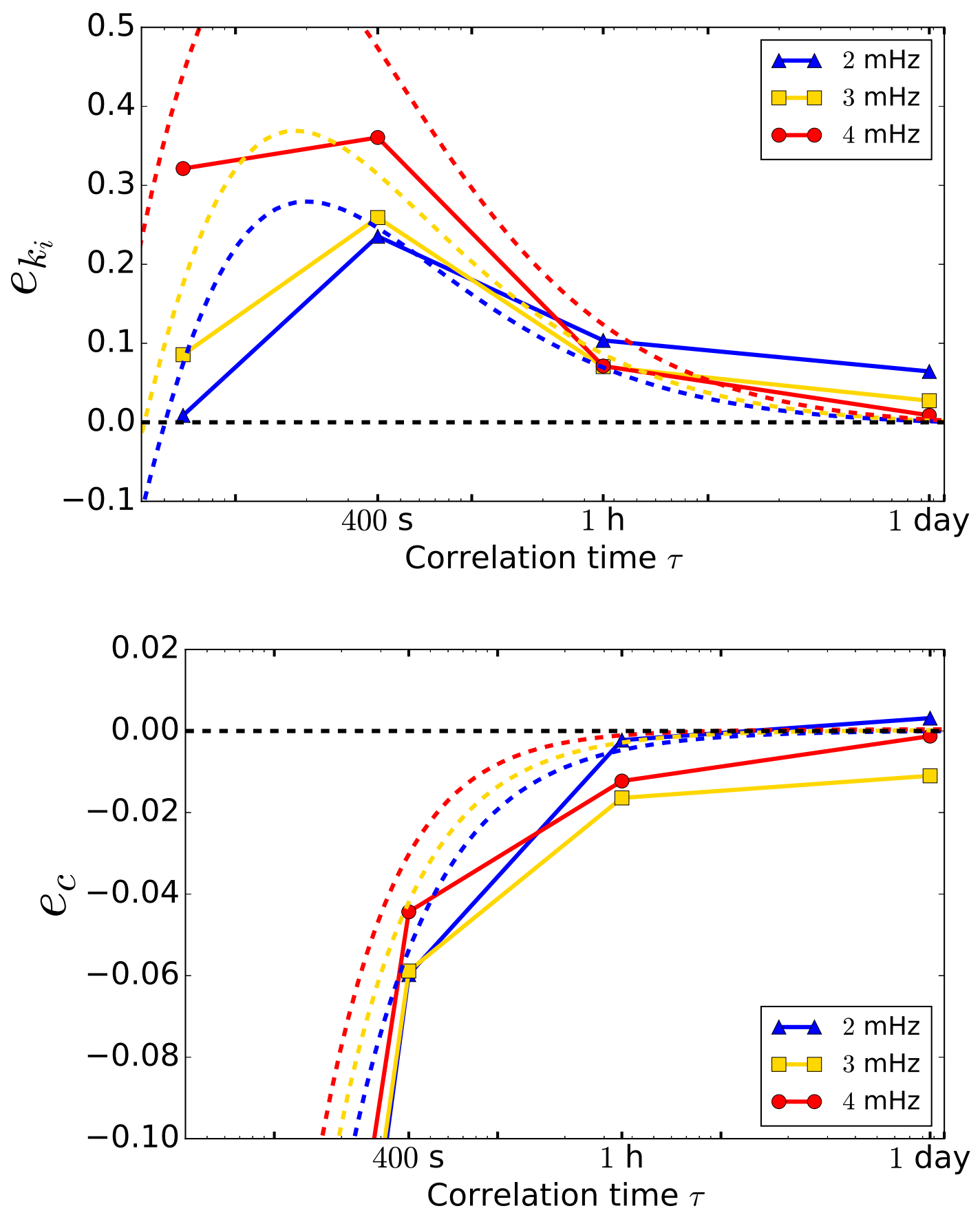

Figure 2.10: Relative error on the attenuation (top) and the effective wave speed difference (bottom) at 2, 3 and $4 \mathrm{mHz}$ (medium 2). The error is between the quantities at $\tau$ and at $\tau \rightarrow \infty$. The dashed lines are the predictions from the time-dependent Keller theory.

small-scale incoherence, so less attenuation. The transition between the two regimes corresponds to a resonance, located according to the theory at about $\tau=195 \mathrm{~s}, \tau=180 \mathrm{~s}$ and $\tau=135 \mathrm{~s}$ at 2,3 and $4 \mathrm{mHz}$. On the other hand, $e_{c}$ being negative, the approximation overestimates the decrease in effective wave speed, because longer-lived features are 
2 Acoustic wave propagation through solar granulation: Validity of effective-medium theories, coda waves

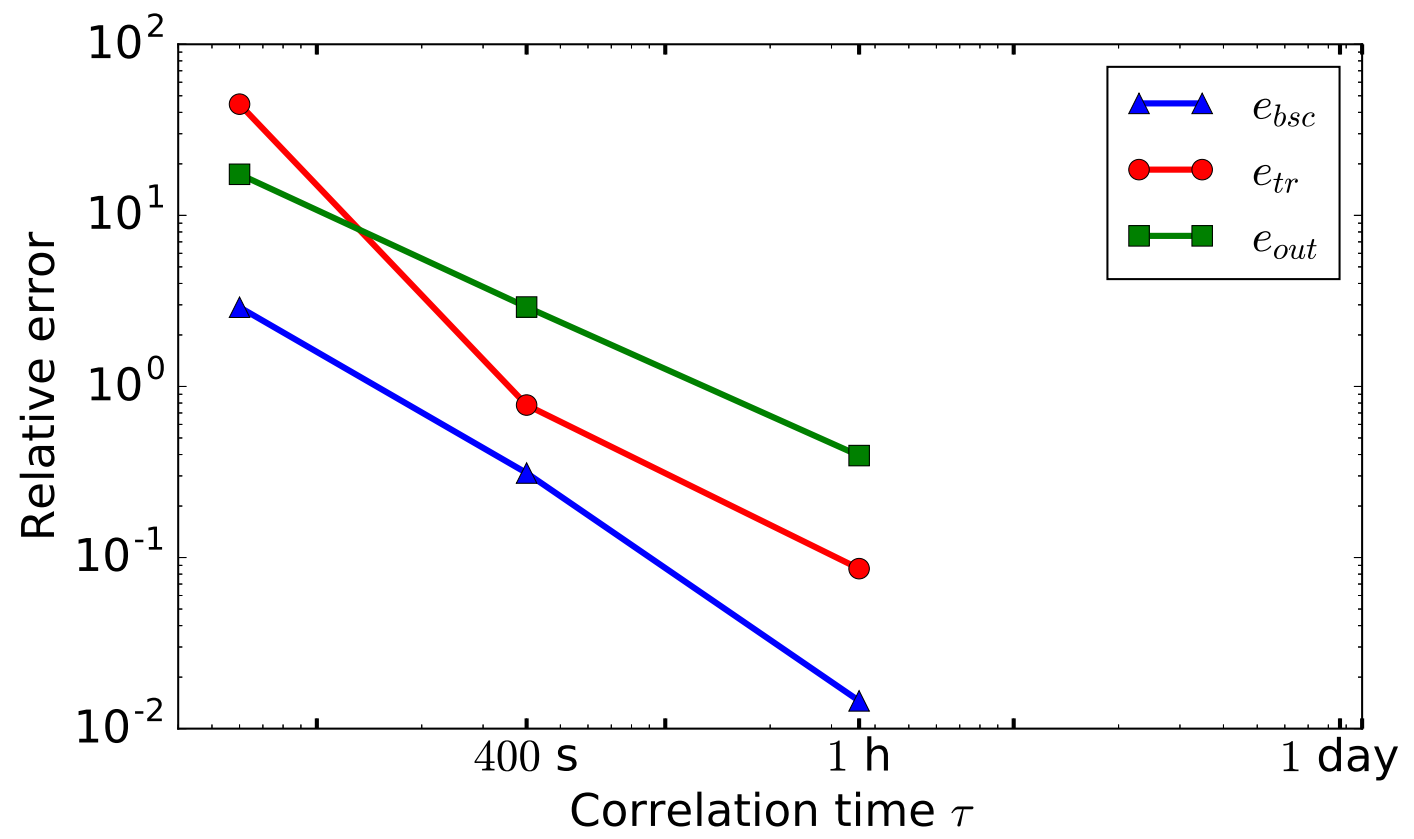

Figure 2.11: Relative error in the variance integrated in space before, in and after the random medium, at $t=8500 \mathrm{~s}$. The error is between the quantities at $\tau$ and at $\tau=1$ day.

better "seen" by the wave packets. The decrease is therefore a monotonic function of $\tau$, with its asymptotic value at $\tau \rightarrow \infty$ only determined by the value of the ratio of the wave number over the typical size of the scatterer. The error is frequency-dependent and, on average over the three central frequencies, is $29 \%$ (respectively $-5 \%$ ) for the attenuation (respectively the effective wave speed difference) at $\tau=400 \mathrm{~s}$.

As for the variance, we assume $\tau=1$ day $\simeq \infty$. This is justified as the propagation time in the random medium of length $30 \mathrm{Mm}$ is about $1 \mathrm{~h} \ll 1$ day. We compute therefore

$$
\begin{aligned}
& e_{b s c}(\tau)=\left(\mathcal{E}_{b s c}(\tau)-\mathcal{E}_{b s c}(1 \text { day })\right) / \mathcal{E}_{b s c}(1 \text { day }) \\
& e_{\text {out }}(\tau)=\left(\mathcal{E}_{\text {out }}(\tau)-\mathcal{E}_{\text {out }}(1 \text { day })\right) / \mathcal{E}_{\text {out }}(1 \text { day }) \\
& e_{t r}(\tau)=\left(\mathcal{E}_{t r}(\tau)-\mathcal{E}_{t r}(1 \text { day })\right) / \mathcal{E}_{t r}(1 \text { day })
\end{aligned}
$$

As shown in Fig. 2.11, the relative errors at $\tau=400 \mathrm{~s}$ are then about 30\%, 80\% and $290 \%$ for the back-scattered, trapped and outgoing variance, respectively. Hence it appears that for medium 2, the variance is more sensitive to the correlation time than the coherent wave field, and that the back-scattered coda is less sensitive than the rest of the variance.

The relative errors for medium 1 at $\tau=400 \mathrm{~s}$ are presented for comparison purposes in Table 2.2. In this case, the frozen-medium approximation overestimates the attenuation. Most of the power is indeed located at large scales, so the attenuation is mostly caused by the large-scale incoherence between the realizations (shifts of the wave packets), which triggers a broadening and damping of the coherent wave packet. Therefore, the impact of scattering is larger if the scatterers persist while the wave packets propagate through them than if the scatterers evolve in time. On the other hand, the frozen-medium approximation still overestimates, albeit by a larger amount, the decrease in effective wave speed. The 
Table 2.2: Relative error at $\tau=400 \mathrm{~s}$ for the measured quantities for media 1 and 2.

\begin{tabular}{cccc} 
& & Medium 1 & Medium 2 \\
\hline \multirow{2}{*}{ Coherent wave } & $e_{k_{i}}$ & $-25 \%$ & $29 \%$ \\
& $e_{c}$ & $-19 \%$ & $-5 \%$ \\
\hline Variance & $e_{b s c}$ & $46 \%$ & $31 \%$
\end{tabular}

$e_{k_{i}}$ and $e_{c}$ are averaged over the three central frequencies 2,3 and $4 \mathrm{mHz}$. They are computed using both the temporal and the frequency codes. On the other hand, since we study the variance in time domain, the errors for this quantity are computed using only the temporal code.

error for both quantities does not depend much on frequency, and is about $-25 \%$ for the attenuation and $-19 \%$ for the effective wave speed. For the back-scattered coda, the error increases to $46 \%$.

\subsection{Discussion}

\subsubsection{Accuracy of the theories}

All theories predict a decrease in the effective wave speed. The effective wave speed and the attenuation of the coherent wave field are best described by the Keller approximation. The Born second-order solution, although consistent with the Keller solution for small perturbations, performs poorly for larger amplitudes, therefore it may not be suited for the study of acoustic wave scattering by solar granulation unless it is on small distances $(<30 \mathrm{Mm})$. The homogenization technique and the geometrical optics do not model the attenuation of the coherent wave field. However they correctly represent the decrease in wave speed for low and high frequencies, respectively.

\subsubsection{Validity of the frozen-medium approximation}

It is more convenient to study acoustic wave propagation in the frequency domain, but this is easily doable only when the coefficients of the wave equation do not depend on time, i.e. when one can use a snapshot of the random medium. As summarized in Table 2.2, we find that for medium 2, the attenuation is underestimated by the frozen-medium approximation by $29 \%$ at the frequencies of interest for the Sun. As for the effective wave speed difference, which is an important quantity since it is directly related to the helioseismic travel times, it is overestimated by $5 \%$. The greater error for $k_{i}$ seemingly arises from the presence of a resonance of the function $k_{i}(\tau)$ at a correlation time close to that of granulation, while the effective wave speed does not exhibit such a feature. We note that the relative error in $c_{\text {eff }}-c_{0}$ is similar to that of $k_{i}$ in medium 1 , when the power of the perturbation is distributed at low scales. The frozen-medium approximation underestimates the variance of the amplitude of back-scattered coda waves by about $30 \%$. 
2 Acoustic wave propagation through solar granulation: Validity of effective-medium theories, coda waves

\subsubsection{Detectability of coda waves}

The numerical simulations show the emergence of coda waves, which are an interesting effect of multiple scattering present both in single realizations of the wave field and in its variance, but not in the coherent wave field. Coda waves are seen trailing the ballistic wave packet, and also as late arrival back-scattered waves (in one dimension). In helioseismology, acoustic waves are measured via the two-point cross-covariance function of the solar oscillations. Therefore, in order to identify coda waves in the Sun, one needs to study the statistical variance of this cross-covariance function.

\section{Acknowledgements}

We thank Aaron C. Birch for useful discussions and comments. PLP is a member of the International Max Planck Research School (IMPRS) for Solar System Science at the University of Göttingen. The computational resources were provided by the German Data Center for SDO through grant 50OL1701 from the German Aerospace Center (DLR). 


\subsection{Appendix}

\subsubsection{Keller approximation: Time-independent random medium}

Starting from a time-independent random medium $c(x)$, we can take the Fourier transport of the wave equation:

$$
\omega^{2} \tilde{\phi}(x, \omega)+\partial_{x}^{2}\left(c^{2}(x) \tilde{\phi}(x, \omega)\right)=0 .
$$

The autocorrelation written in Eq. (2.2) can be simplified to

$$
\left\langle\delta c(x) \delta c\left(x^{\prime}+x\right)\right\rangle=c_{0}^{2} \epsilon^{2} f(x) .
$$

For clarity, we drop the argument $\omega$ in the expression of $\tilde{\phi}$.

Keller (1964) considers an unbounded spatially random medium and assumes statistical homogeneity, isotropy and stationarity. The calculation could be generalized to the case of a localized perturbation, however we follow the original derivation. It does accurately model our problem since the amplitude attenuation and the effective wave speed shift arise because of the perturbed region. Therefore only the boundary effects are not taken into account. Keller made the first part of his derivation in time-domain, using the fact that the Green's function for the 3D wave equation is essentially a delta function, which simplifies the calculation. In 1D however, the Green's function is related to the Heaviside step function. We shall first derive the Keller solution in frequency domain for a frozen medium, then generalize in Appendix 2.7.2 to the solution in time domain.

The wave equation given by Eq. (2.33) can be written as

$$
\left(\tilde{L}_{0}+\tilde{L}_{1}+\tilde{L}_{2}\right) \tilde{\phi}=0
$$

where

$$
\begin{aligned}
\tilde{L}_{0} \tilde{\phi} & =\omega^{2} \tilde{\phi}+c_{0}^{2} \partial_{x}^{2} \tilde{\phi}, \\
\tilde{L}_{1} \tilde{\phi} & =2 c_{0} \partial_{x}^{2}(\delta c(x) \tilde{\phi}), \\
\tilde{L}_{2} \tilde{\phi} & =\partial_{x}^{2}\left(\delta c^{2}(x) \tilde{\phi}\right) .
\end{aligned}
$$

The unperturbed equation, assuming a constant background sound speed, is

$$
\tilde{L}_{0} \tilde{\phi}_{0}=0
$$

The corresponding Green's function $G_{0}$, solution of $\tilde{L}_{0} \tilde{G}_{0}\left(x, x^{\prime}\right)=\delta\left(x-x^{\prime}\right)$ where $\delta$ is the Dirac delta function, is

$$
\tilde{G}_{0}\left(x, x^{\prime}\right)=-\frac{i}{2 c_{0}^{2} k_{0}} e^{i k_{0}\left|x-x^{\prime}\right|},
$$

where $k_{0}=\omega / c_{0}$. Keller has shown that one can find a new wave equation for the coherent wave field under the form

$$
\left(\tilde{L}_{0}-\left\langle\tilde{L}_{1} \tilde{L}_{0}^{-1} \tilde{L}_{1}\right\rangle+\left\langle\tilde{L}_{2}\right\rangle\right)\langle\tilde{\phi}\rangle=0
$$


2 Acoustic wave propagation through solar granulation: Validity of effective-medium theories, coda waves

with

$$
\begin{aligned}
& \left(\left\langle\tilde{L}_{1} \tilde{L}_{0}^{-1} \tilde{L}_{1}\right\rangle\langle\tilde{\phi}\rangle\right)(x) \\
& =\left\langle c_{0} \partial_{x}^{2}\left(2 \delta c(x) \int_{-\infty}^{\infty} \mathrm{d} x^{\prime} \tilde{G}_{0}\left(x, x^{\prime}\right) c_{0} \partial_{x^{\prime}}^{2}\left[2 \delta c\left(x^{\prime}\right)\left\langle\tilde{\phi}\left(x^{\prime}\right)\right\rangle\right]\right)\right\rangle \\
& =4 c_{0}^{4} \epsilon^{2} \partial_{x}^{2}\left(\int_{-\infty}^{\infty} \mathrm{d} x^{\prime} \tilde{G}_{0}\left(x, x^{\prime}\right) \partial_{x^{\prime}}^{2}\left[f\left(x^{\prime}-x\right)\left\langle\tilde{\phi}\left(x^{\prime}\right)\right\rangle\right]\right) .
\end{aligned}
$$

We assume that the coherent wave field also satisfies a wave equation with a complex wave number $k$ so that

$$
\left\langle\tilde{\phi}\left(x^{\prime}\right)\right\rangle=e^{i k x^{\prime}}
$$

In this case,

$$
\partial_{x^{\prime}}^{2}\left[f\left(x^{\prime}-x\right)\left\langle\tilde{\phi}\left(x^{\prime}\right)\right\rangle\right]=\left[\left(\partial_{x^{\prime}}+i k\right)^{2} f\left(x^{\prime}-x\right)\right] e^{i k x^{\prime}}
$$

Therefore,

$$
\begin{aligned}
\left(\left\langle\tilde{L}_{1} \tilde{L}_{0}^{-1} \tilde{L}_{1}\right\rangle\langle\tilde{\phi}\rangle\right)(x) & =4 c_{0}^{4} \epsilon^{2} \partial_{x}^{2}\left(e^{i k x} I(x)\right) \\
& =4 c_{0}^{4} \epsilon^{2}\left(\left(\partial_{x}+i k\right)^{2} I(x)\right)\langle\tilde{\phi}(x)\rangle,
\end{aligned}
$$

where

$$
I(x)=\int_{-\infty}^{\infty} \mathrm{d} x^{\prime} \tilde{G}_{0}\left(x, x^{\prime}\right)\left[\left(\partial_{x^{\prime}}+i k\right)^{2} f\left(x^{\prime}-x\right)\right] e^{i k\left(x^{\prime}-x\right)} .
$$

On the other hand,

$$
\left\langle\tilde{L}_{2}\right\rangle\langle\tilde{\phi}\rangle=-c_{0}^{2} \epsilon^{2} k^{2}\langle\tilde{\phi}\rangle .
$$

Using Eqs. (2.45) and (2.47) in Eq. (2.41), the perturbed wave equation for the coherent wave field is

$$
\left(\partial_{x}^{2}+k_{0}^{2}-4 c_{0}^{2} \epsilon^{2}\left(\partial_{x}+i k\right)^{2} I(x)-\epsilon^{2} k^{2}\right)\langle\tilde{\phi}(x)\rangle=0 .
$$

We can define the complex wave number by

$$
k^{2}=k_{0}^{2}-4 c_{0}^{2} \epsilon^{2}\left(\partial_{x}+i k\right)^{2} I(x)-\epsilon^{2} k^{2} .
$$

Since the autocorrelation function of the perturbation depends here only on the difference $x^{\prime}-x, I(x)=I$. In the small-perturbation approximation, one can also replace $k$ by $k_{0}$ in the right-hand term, to get finally

$$
k^{2}=k_{0}^{2}\left(1+4 c_{0}^{2} \epsilon^{2} I-\epsilon^{2}\right) .
$$

We note that it is possible to keep $k$ in the right-hand side, one then has to solve a biquadratic complex equation. Here we only use the approximation.

In this paper, we used in one case an exponential correlation function

$$
f_{1}\left(x^{\prime}-x\right)=f_{1}(\zeta)=\epsilon^{2} e^{-|\zeta| / a}
$$

where $\zeta=x^{\prime}-x$. In this case $\partial_{\zeta} f_{1}(\zeta)=-\operatorname{sign}(\zeta) f_{1}(\zeta) / a$ and $\partial_{\zeta}^{2} f_{1}(\zeta)=f_{1}(\zeta) / a^{2}-\frac{2}{a} \delta(\zeta)$, so that

$$
I=-\frac{i}{2 c_{0}^{2} k_{0} a}\left(2 i k_{0} a-\left(k_{0} a\right)^{2}+\frac{\left(k_{0} a\right)^{2}}{2 i k_{0} a-1}\right) .
$$


Thus

$$
\begin{aligned}
k^{2}= & k_{0}^{2}+\epsilon^{2} k_{0}^{2}\left(3-\frac{4\left(k_{0} a\right)^{2}}{1+4\left(k_{0} a\right)^{2}}\right) \\
& +2 i \epsilon^{2} k_{0}^{2}\left(k_{0} a+\frac{k_{0} a}{1+4\left(k_{0} a\right)^{2}}\right) .
\end{aligned}
$$

This formula gives the damping $\operatorname{Im}(k)=k_{i}$ of the coherent wave $\langle\tilde{\phi}\rangle$ and the effective wave speed $\omega / \operatorname{Re}(k)=c_{\text {eff }}$ of the medium. For medium 2, we evaluate the integral numerically.

\subsubsection{Keller approximation: Time-dependent random medium}

Here, we extend the previous analysis to a time-dependent random medium $c(x, t)$. We rewrite the problem as follows:

$$
\left(L_{0}+L_{1}+L_{2}\right) \phi=0
$$

where

$$
\begin{aligned}
& L_{0} \phi=-\partial_{t}^{2} \phi+c_{0}^{2} \partial_{x}^{2}(\phi), \\
& L_{1} \phi=2 c_{0} \partial_{x}^{2}(\delta c(x, t) \phi), \\
& L_{2} \phi=\partial_{x}^{2}\left(\delta c(x, t)^{2} \phi\right) .
\end{aligned}
$$

The associated Green's function, solution of $L_{0} G_{0}\left(t, t^{\prime}, x, x^{\prime}\right)=\delta\left(t-t^{\prime}\right) \delta\left(x-x^{\prime}\right)$, is

$$
G_{0}\left(x, x^{\prime}, t, t^{\prime}\right)=-\frac{1}{2 c_{0}} \Theta\left(c_{0}\left(t-t^{\prime}\right)-\left|x-x^{\prime}\right|\right),
$$

where $\Theta$ is the Heaviside step function. With these new operators, writing the wave field as

$$
\langle\phi(x, t)\rangle=e^{i(k x-\omega t)},
$$

it follows that

$$
\begin{aligned}
& \left(\left\langle L_{1} L_{0}^{-1} L_{1}\right\rangle\langle\phi\rangle\right)(x, t) \\
& =-4 c_{0}^{4} \epsilon^{2} \partial_{x}^{2} \iint_{-\infty}^{\infty} \mathrm{d} x^{\prime} \mathrm{d} t^{\prime} G_{0}\left(x, x^{\prime}, t, t^{\prime}\right) \times \\
& \partial_{x^{\prime}}^{2}\left[f\left(x^{\prime}-x\right) g\left(t^{\prime}-t\right)\left\langle\phi\left(t^{\prime}, x^{\prime}\right)\right\rangle\right]
\end{aligned}
$$

and

$$
\left\langle L_{2}\right\rangle\langle\phi\rangle=c_{0}^{2} \epsilon^{2} k^{2}\langle\phi\rangle .
$$

The calculations are similar to those for the time-independent random medium. Replacing again $k$ by $k_{0}$ in the $O\left(\epsilon^{2}\right)$ terms, one gets for medium 1

$$
k^{2}=k_{0}^{2}\left(1-\epsilon^{2}\left[1+2 c_{0} \frac{\tau}{a} \frac{1}{Q 1}\left(-2+\frac{Q_{2}}{Q_{3}}-\frac{Q_{4}}{Q_{5}}\right)\right]\right),
$$


2 Acoustic wave propagation through solar granulation: Validity of effective-medium theories, coda waves

where

$$
\begin{aligned}
Q_{1} & =1-i \omega \tau, \\
Q_{2} & =\left(1-i k_{0} a\right)^{2}, \\
Q_{3} & =1-i k_{0} a+Q_{1} \frac{a}{\tau c_{0}}, \\
Q_{4} & =\left(1+i k_{0} a\right)^{2}, \\
Q_{5} & =-1-i k_{0} a-Q_{1} \frac{a}{\tau c_{0}} .
\end{aligned}
$$

We have demonstrated here the possibility to develop a time-dependent theory given the knowledge of the power spectrum (or autocorrelation function) of the perturbation. We note that here too, the solution for medium 2 presented in the corpus is evaluated numerically.

\subsubsection{Second-order Born approximation}

Another theory is the second-order Born approximation, which we derive here for a timeindependent random medium $c(x)$. It is similar to the Keller theory, but one does not look for an effective wave equation satisfied by the mean wave field. Instead, one writes the mean wave field as a series up to a certain order, each term being proportional to a power of $\epsilon$. Using the same notations for the operators as in Appendix 2.7.1, denoting $\tilde{\phi}_{0}$ the unperturbed wave field and $\tilde{\phi}_{1}$ the correction such that $\tilde{\phi}=\tilde{\phi}_{0}+\tilde{\phi}_{1}$, the 1st-order Born approximation reads

$$
\tilde{\phi}=\tilde{\phi}_{0}-\tilde{L}_{0}^{-1} \tilde{L}_{1} \tilde{\phi}_{0}+O\left(\epsilon^{2}\right) .
$$

Taking the average, one gets $\langle\tilde{\phi}\rangle=\tilde{\phi}_{0}+O\left(\epsilon^{2}\right)$. This means that we have to go down to the second order:

$$
\tilde{\phi}=\tilde{\phi}_{0}-\tilde{L}_{0}^{-1} \tilde{L}_{1} \tilde{\phi}_{0}+\tilde{L}_{0}^{-1} \tilde{L}_{1} \tilde{L}_{0}^{-1} \tilde{L}_{1} \tilde{\phi}_{0}-\tilde{L}_{0}^{-1} \tilde{L}_{2} \tilde{\phi}_{0}+O\left(\epsilon^{3}\right)
$$

which, averaged, gives

$$
\langle\tilde{\phi}\rangle=\tilde{\phi}_{0}+\tilde{L}_{0}^{-1}\left\langle\tilde{L}_{1} \tilde{L}_{0}^{-1} \tilde{L}_{1}\right\rangle \tilde{\phi}_{0}-\tilde{L}_{0}^{-1}\left\langle\tilde{L}_{2}\right\rangle \tilde{\phi}_{0}+O\left(\epsilon^{3}\right) .
$$

We can compute $\left\langle\tilde{L}_{1} \tilde{L}_{0}^{-1} \tilde{L}_{1}\right\rangle \tilde{\phi}_{0}$ and $\left\langle\tilde{L}_{2}\right\rangle \tilde{\phi}_{0}$ easily because these are mostly equations 2.42 and 2.47 replacing $\langle\tilde{\phi}(x)\rangle=e^{i k x}$ by $\tilde{\phi}_{0}(x)=e^{i k_{0} x}$. One finally needs to apply $\tilde{L}_{0}^{-1}$ which is a convolution by the Green's function. In order to converge, the integration requires a compact support. To model the localization of the perturbation between $X$ and $X+L$, we introduce the window function

$$
w(\bar{x})=\Theta(\bar{x}-X)-\Theta(\bar{x}-(X+L))
$$

where $\bar{x}=\left(x+x^{\prime}\right) / 2$, so that

$$
\left\langle\delta c(x) \delta c\left(x^{\prime}\right)\right\rangle=c_{0}^{2} \epsilon^{2} e^{-|\zeta| / a} w(\bar{x}) .
$$

The approximate solution in $[X, X+L]$ is

$$
\langle\tilde{\phi}(x)\rangle \simeq \tilde{\phi}_{0}(x)\left(1+\epsilon^{2}\left[\frac{3}{2} i k_{0} a-\left(k_{0} a\right)^{2}+\frac{\left(k_{0} a\right)^{2}}{2 i k_{0} a-1}\right](x-X)\right),
$$


which, since $\epsilon \ll 1$, can be written (omitting a phase term) in the form $\langle\tilde{\phi}(x)\rangle \simeq \mathrm{e}^{i k(x-X)}$ where $k$ has the same expression as for the Keller theory (Eq. (2.53)). To this level of approximation, the effective $k$ does not depend on $L$.

\subsubsection{Spatial homogenization}

In order to perform the spatial homogenization for a time-independent random medium $c(x)$, we consider the variable

$$
\psi=c^{2} \phi
$$

which is solution of

$$
\partial_{t}^{2} \frac{\psi}{c^{2}}-\partial_{x}^{2} \psi=0
$$

Multiplying the equation by $\partial_{t} \psi$ and integrating over space, then applying an integration by parts, we find that

$$
\partial_{t} \mathcal{E}=0
$$

where

$$
\mathcal{E}=\int \mathrm{d} x\left(\frac{1}{2 c^{2}}\left(\partial_{t} \psi\right)^{2}+\left(\partial_{x} \psi\right)^{2}\right)
$$

is an expression for the energy. Since it is invariant, we are certain that the homogenization expansion converges.

The medium is assumed to vary on length scales much shorter than the wave (for solar granulation the length scale $a$ is at least shorter than the wave length of acoustic waves). We moreover assume the periodicity of the medium: $c(x)=c(x+a)$. We separate the spatial variable $x$ into $y_{0}$, a slow-varying spatial scale, and $y_{1}=y_{0} / \eta$, a fast-varying spatial scale, where $\eta=k_{0}\left(\omega_{0}\right) a \ll 1$ (e.g., Hanasoge et al. 2013). Then

$$
\psi=\psi\left(y_{0}, y_{1}, t\right)
$$

and

$$
\begin{aligned}
& \partial_{x}=\partial_{y_{0}}+\frac{1}{\eta} \partial_{y_{1}}, \\
& \partial_{x}^{2}=\partial_{y_{0}}^{2}+\frac{2}{\eta} \partial_{y_{1}} \partial_{y_{0}}+\frac{1}{\eta^{2}} \partial_{y_{1}}^{2} .
\end{aligned}
$$

We also expand the solution

$$
\psi=\psi_{0}+\eta \psi_{1}+\eta^{2} \psi_{2}+O\left(\eta^{3}\right)
$$

where $\psi_{i}=\psi_{i}\left(y_{0}, y_{1}, t\right)=\psi_{i}\left(y_{0}, y_{1}+a, t\right)$. We can now proceed to solving the equation order by order. Order $\eta^{-2}$ gives

$$
\partial_{y_{1}}^{2} \psi_{0}=0 .
$$

Multiplying by $\psi_{0}$, integrating over $y_{1}$ and using the argument of periodicity, one gets

$$
\int_{0}^{a}\left(\partial_{y_{1}} \psi_{0}\right)^{2} \mathrm{~d} y_{1}=0
$$


2 Acoustic wave propagation through solar granulation: Validity of effective-medium theories, coda waves

meaning that $\psi_{0}$ does not depend on $y_{1}$. Order $\eta^{-1}$ then gives

$$
\partial_{y_{1}}^{2} \psi_{1}=0
$$

meaning that $\psi_{1}$ does not depend on $y_{1}$ either. Finally, at order $\eta^{0}$,

$$
\partial_{t}^{2} \frac{\psi_{0}}{c^{2}}-\partial_{y_{0}}^{2} \psi_{0}-\partial_{y_{1}}^{2} \psi_{2}=0
$$

Integrating over the fast-varying coordinate $y_{1}$, invoking periodicity, one finds the following homogenized equation for $\psi$ :

$$
\partial_{t}^{2} \psi_{0}-\frac{1}{\overline{c^{-2}}} \partial_{y_{0}}^{2} \psi_{0}=0
$$

where $\overline{c^{-2}}=\frac{1}{a} \int_{0}^{a} c^{-2} \mathrm{~d} y_{1}$ is a spatial average. The homogenization method, used here for a periodic medium, has been generalized to a statistically homogeneous and ergodic random medium, by making the period tend to $\infty$ (e.g., Papanicolaou and Varadhan 1982). The spatial average identifies then with the statistical average. The homogenized sound speed $c_{\mathrm{h}}$ of the medium is therefore equal to $\left\langle c^{-2}\right\rangle^{-1 / 2}$. Knowing that $c=c_{0}+\delta c, c^{-2} \simeq$ $c_{0}^{-2}\left(1-2 \delta c / c_{0}+3 \delta c^{2} / c_{0}^{2}\right)$ and $\left\langle c^{-2}\right\rangle \simeq c_{0}^{-2}\left(1+3 \epsilon^{2}\right)$. Hence:

$$
c_{\mathrm{h}}=\left\langle c^{-2}\right\rangle^{-1 / 2} \simeq c_{0}\left(1-\frac{3}{2} \epsilon^{2}\right)
$$

We note that the spatial homogenization technique does not make an attenuation arise.

\subsubsection{Ray approximation}

The geometrical optics theory, or ray theory, is an infinite-frequency approximation. In practice the applicability conditions are (Rytov et al. 1989a):

$$
\begin{aligned}
\epsilon & \ll 1, \\
k_{0} a & \gg 1, \\
k_{0} a & \gg 2 \pi \frac{L}{a} .
\end{aligned}
$$

Under these conditions, the wave travel time inside the random medium starting at $x=X$ is computed as an integral of the slowness over the ray path:

$$
t=\int_{X}^{x} c^{-1}(s) \mathrm{d} s=(x-X) \overline{c^{-1}}
$$

Assuming ergodicity of the random medium, the spatial average identifies with the statistical average and

$$
c_{\text {ray }}=\left\langle c^{-1}\right\rangle^{-1} \text {. }
$$




\subsubsection{Comparing theories with numerical simulations in the limit $\epsilon \rightarrow$ 0}

Fig. 2.12 summarizes the accuracy of the (frozen) Keller theory, the Born second-order approximation, the spatial homogenization and the ray theory in the small-perturbation regime $(\epsilon=0.01)$. For each simulation, ten sets of $10^{4}$ realizations were generated to get the error bars. For such a small perturbation, we are in the regime of validity of the Born and Keller theories and the results are in agreement with the numerical simulations for the attenuation and the effective wave speed. The attenuation for medium 2 resulting from the time-domain simulation differs from the attenuation from the frequency-domain one, likely because of numerical diffusion. As $k_{0} a$ is of order unity in our setup, we are not a priori in the regime of validity of the homogenization or the geometrical optic theories. However, the geometrical optics is in good agreement with the numerical simulations for medium 1, despite the fact that the condition $k_{0} a \gg 2 \pi \frac{L}{a}$ is not verified in our simulations. Medium 2 exhibits, just like for $\epsilon=0.1$, a transition from the homogenization regime at small frequencies $(<1 \mathrm{mHz})$ to the geometrical optics regime at high frequencies $(>5 \mathrm{mHz})$. 
2 Acoustic wave propagation through solar granulation: Validity of effective-medium theories, coda waves
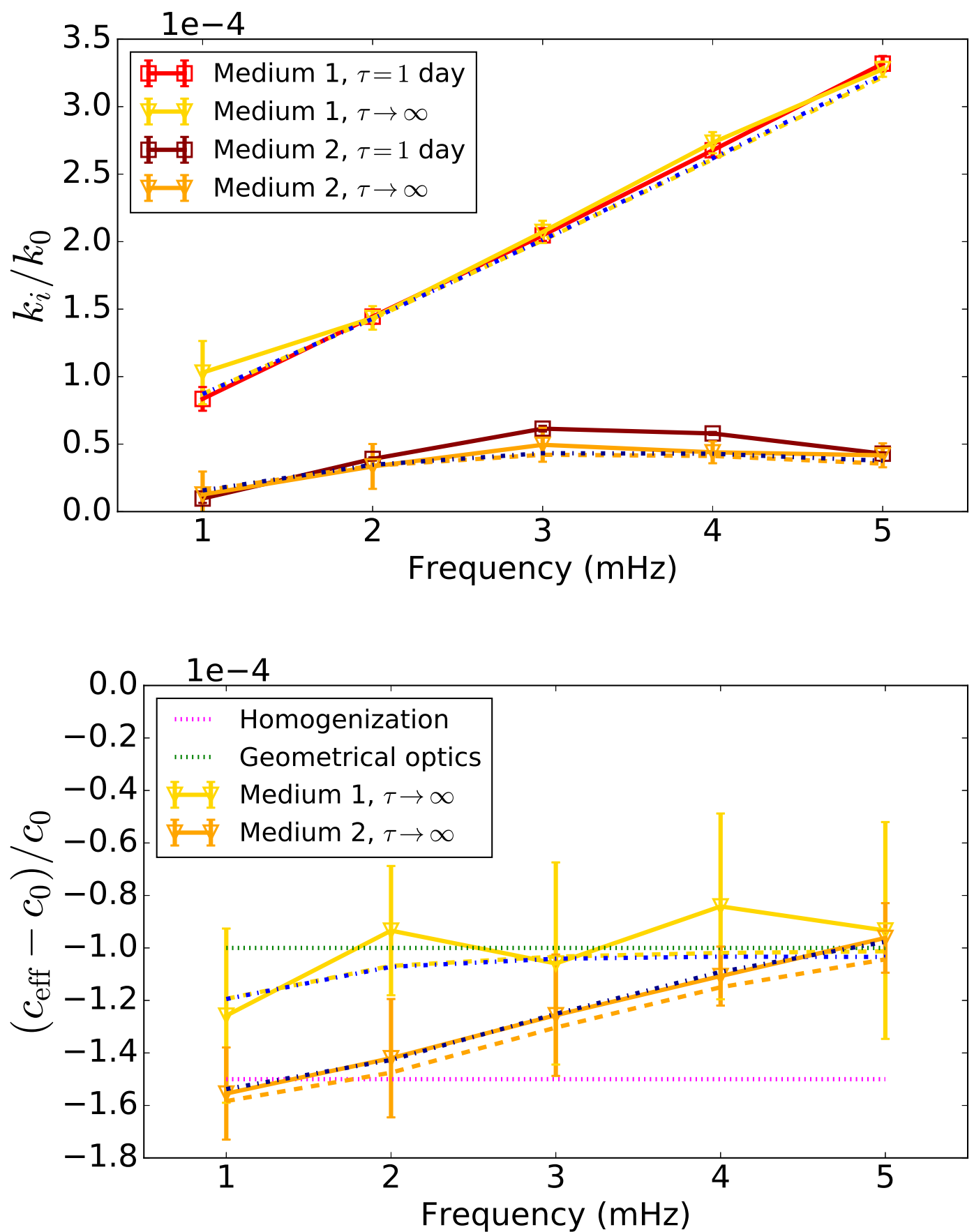

Figure 2.12: Comparison of theories with simulations for the average wave field $(\epsilon=$ 0.01). Top: attenuation. Bottom: effective wave speed. The triangles are for the simulations in frequency domain $(\tau \rightarrow \infty)$, the squares for those in time domain $(\tau=1$ day). The two dashed-dotted blue lines are the Born solutions for media 1 and 2, while the yellow and orange dashed lines are the Keller solutions. $1-\sigma$ error bars are shown. 


\title{
3 Contribution of flows around active regions to the north-south helioseismic measurements ${ }^{1}$
}

\begin{abstract}
Context. In local helioseismology, the travel times of acoustic waves propagating in opposite directions along the same meridian inform us about horizontal flows in the northsouth direction. The longitudinal averages of the north-south helioseismic travel-time shifts vary with the sunspot cycle.

Aims. We aim to study the contribution of inflows into solar active regions to this solar-cycle variation.

Methods. To do so, we identify the local flows around active regions in the horizontal flow maps obtained from correlation tracking of granulation in SDO/HMI continuum images. We compute the forward-modeled travel-time perturbations caused by these inflows using 3D sensitivity kernels. In order to compare with the observations, we average these forward-modeled travel-time perturbations over longitude and time in the same way as the measured travel times.

Results. The forward-modeling approach shows that the inflows associated with active regions may account for only a fraction of the solar-cycle variations in the north-south travel-time measurements.

Conclusions. The travel-time perturbations caused by the large-scale inflows surrounding the active regions do not explain in full the solar-cycle variations seen in the helioseismic measurements of the meridional circulation.
\end{abstract}

\subsection{Introduction}

The Sun's meridional flow at the surface is poleward with a maximum amplitude of about $15 \mathrm{~m} / \mathrm{s}$ (Duvall 1979). The meridional ciculation, both at the surface and in the deep convection zone, is believed to be a key ingredient in flux-transport dynamo models (e.g., Wang et al. 1991, Dikpati and Gilman 2006, 2009). Observationally, the meridional circulation is given as the longitudinal average of the north-south flows. This longitudinal

\footnotetext{
${ }^{1}$ Contribution statement: P.-L. P. performed research, measured the flow residuals, and wrote the results with contributions from Zhi-Chao Liang, Damien Fournier and Laurent Gizon. Zhi-Chao Liang provided the remapping and averaging codes. Damien Fournier computed the 3D sensitivity kernels.
} 
average is not constant in time: its amplitude and latitudinal dependence change over the solar cycle (e.g., Komm et al. 1993, Hathaway and Rightmire 2010, 2011). It has been proposed that extended inflows around solar active regions (Gizon et al. 2001) modulate the meridional flow at the surface (Gizon 2004, Gizon et al. 2010). These inflows have amplitudes of up to $50 \mathrm{~m} / \mathrm{s}$ near the surface and extend up to $10^{\circ}$ from the center of the active regions or further (Gizon et al. 2001, Hindman et al. 2003, Haber et al. 2004, Hindman et al. 2009, Braun 2019, Gottschling et al. 2021).

Helioseismic travel-time shifts in the north-south direction are sensitive to the meridional flow (e.g., Giles et al. 1997). The measurements made by Gizon et al. (2020) show a solar-cycle modulation. We aim to determine how much of this modulation may indeed be attributed to the near-surface active-region flows. To this end, we isolate and measure the active-region flows in local correlation tracking (LCT) flow maps. By assuming a depth dependence of these flows, we compute forward helioseismic travel-time perturbations in the north-south direction to estimate their contribution to the fluctuations seen in the time-distance measurements. Section 3.2 presents the data and the method. Section 3.3 shows the latitudinal and longitudinal components of the resulting active-region flows. We compute in Section 3.4 the forward helioseismic travel-time perturbations associated with the latitudinal inflows, using 3D Born sensitivity kernels. We attempt to model the inflows with a simple model based on the latitudinal gradient of the unsigned magnetic field in Section 3.5, in order to extend to the previous solar cycle. We compare our results with helioseismic measurements in Section 3.6.

\subsection{Horizontal flows from granulation tracking}

\subsubsection{Flow maps from LCT}

We use the horizontal flow maps computed by Löptien et al. (2017). The original data set covered the period from May 2010 to April 2016, and has later been extended till April 2019 (Gottschling et al. 2021). The maps were obtained by using the Fourier Local Correlation Tracking code (Welsch et al. 2004, Fisher and Welsch 2008). The code tracked pairs of consecutive full-resolution intensity images from SDO/HMI, so that the flows represent the surface motions of solar granulation. The cadence of the flow maps is 30 minutes.

The data contained systematics like the orbital motions of SDO and the shrinking-Sun effect (Lisle and Toomre 2004, Löptien et al. 2016). Löptien et al. (2017) decomposed the flow maps into Zernike polynomials and filtered the time series of the coefficient amplitudes to remove the periods of $24 \mathrm{~h}, 1$ year, and their corresponding harmonics, as well as the zero frequency.

The resulting data product contains the time-varying part of the rotation (torsional oscillations) and of the meridional circulation, plus potentially residual systematics (Gottschling et al. 2021). The velocities are in CCD-frame units (pixels per second). The size of these filtered images is $1024 \times 1024$ pixels; we perform a $5 \times 5$ binning on these images, yielding a spatial resolution of about 10 arcsec per pixel which corresponds to 0.6 heliographic degree per pixel at disk center. 


\subsubsection{Construction of background in CCD frame}

Gottschling et al. (2021) reported that the LCT flow maps contained residual systematics. This means that large-scale background flows must be removed from the flow maps in order to measure the flows related to active regions. These systematics likely depend on the position on the visible disk, in particular if they are a residual of the shrinking-Sun effect. Therefore, the correction should be done in the CCD frame.

\subsubsection{Contour around active regions}

In order to identify regions of magnetic activity, we use the HMI magnetograms at the same time steps as the LCT data. We assume that the magnetic field is purely radial and thus divide the line-of-sight magnetic field $B_{\mathrm{LOS}}$ by the cosine of the great-circle angular distance to disk center to obtain $B_{r}$.

We aim to define a contour around active regions based on a spatially-smoothed magnetic field, in order for the contour itself to be smooth. We use a 2D Gaussian kernel to smooth $\left|B_{r}\right|$, with a full width at half maximum (FWHM) of $10^{\circ}$ (here, $1^{\circ}$ is 1 heliographic degree) because it is the typical horizontal extent of the flows into active regions (see Fig. 3.10). We then define the contour around the active regions as the line along which the smoothed $\left|B_{r}\right|$ is equal to a magnetic threshold $b$, that we choose equal to $3.5 \mathrm{G}$. By aligning the centers of active regions with a total magnetic flux above $10^{21} \mathrm{Mx}$ in a way similar to Braun (2019), we indeed found that this choice allows us to capture flows as far as $12^{\circ}$ away from the active region centers (see Appendix 3.8.1). The contour captures not only the active-region flows, but also the flows around the diffuse flux that is far less concentrated, as seen in Fig. 3.1.

\subsubsection{Background flows}

We consider the flows outside the contour as the background flows which consist of the potential systematics. We generate the background flow maps by computing monthly averages of the flows using only the pixels outside the contour.

The monthly background flow maps can contain little to no data at mid-latitudes during the solar maximum. Therefore, we smooth in time with a Gaussian of full width at half maximum of one year. This width is large enough that it smooths out the regions containing no data for several months, while the background is not expected to vary significantly on that time scale (see Appendix 3.8.2). We further smooth in space with a 2D Gaussian of full width at half maximum of ten pixels, corresponding to an angular distance of $6^{\circ}$ at disk center.

These smoothed background flows exhibit a modulation on the time scale of a solar cycle. This modulation is consistent with what is described by Gottschling et al. (2021). They are studied in more detail in Appendix 3.8.2.

\subsubsection{Active-region flows}

The flows inside the contour are the superposition of the background flows and the activeregion flows. We therefore subtract the smoothed background flows from the 30-minute cadence flow maps; the remaining flows are the active-region flows. 
3 Contribution of flows around active regions to the north-south helioseismic measurements

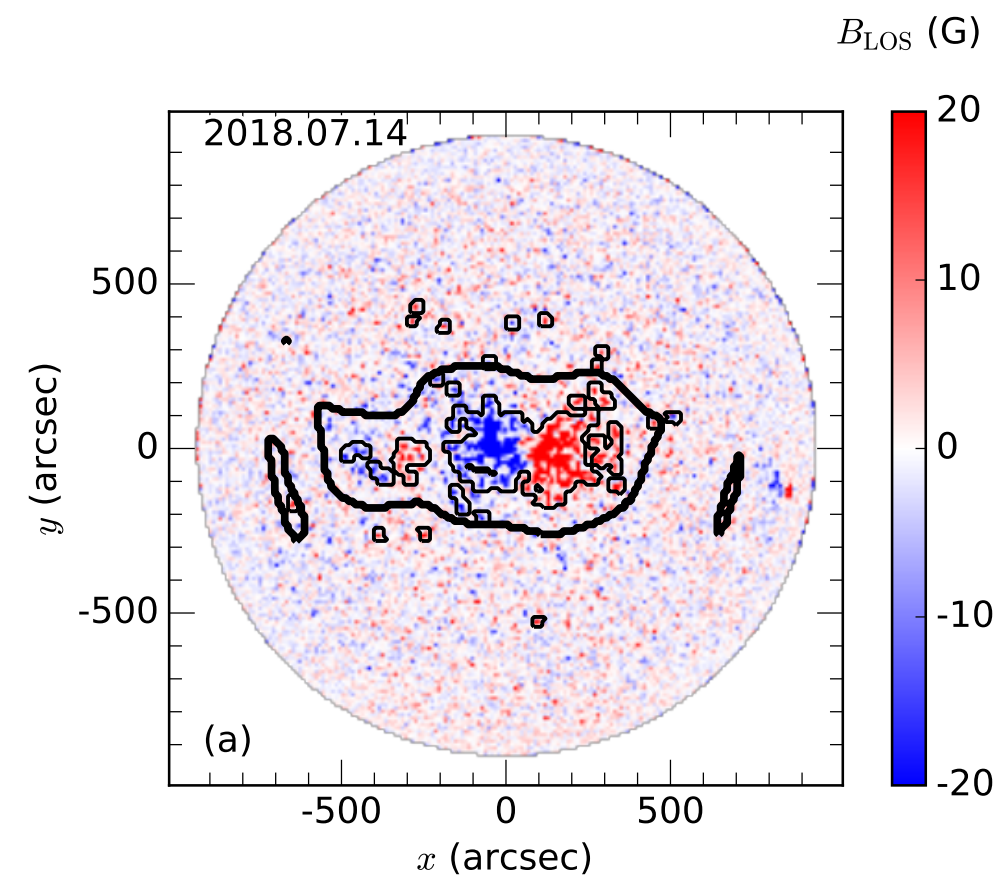

$B_{\mathrm{LOS}}(\mathrm{G})$

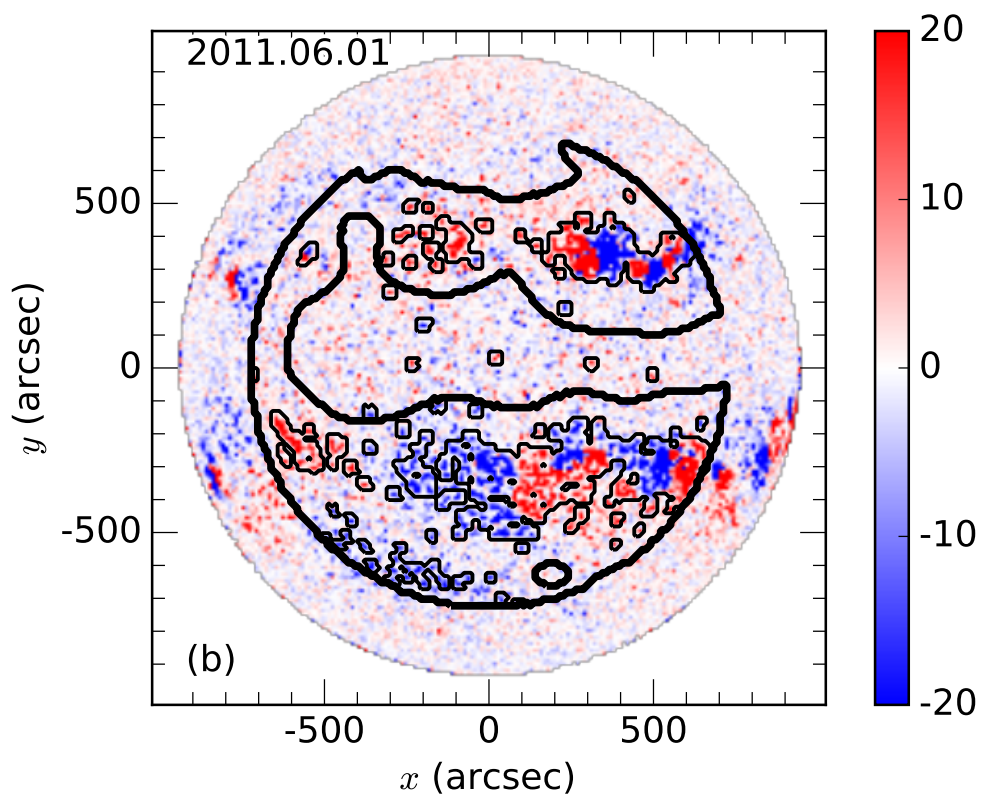

Figure 3.1: Contours (thick black lines) on a quiet day July 14, 2018 (panel a) and on an active day June 1, 2011 (panel $b$ ), superimposed on SDO/HMI magnetograms (lineof-sight magnetic field). The thin black lines represent the contour of the magnetic mask discussed in Section 3.4.2. We only consider pixels whose great-circle distance from disk center is less than $50^{\circ}$, and hence the active region in panel $b$ located close to the limb is not included in the analysis. 


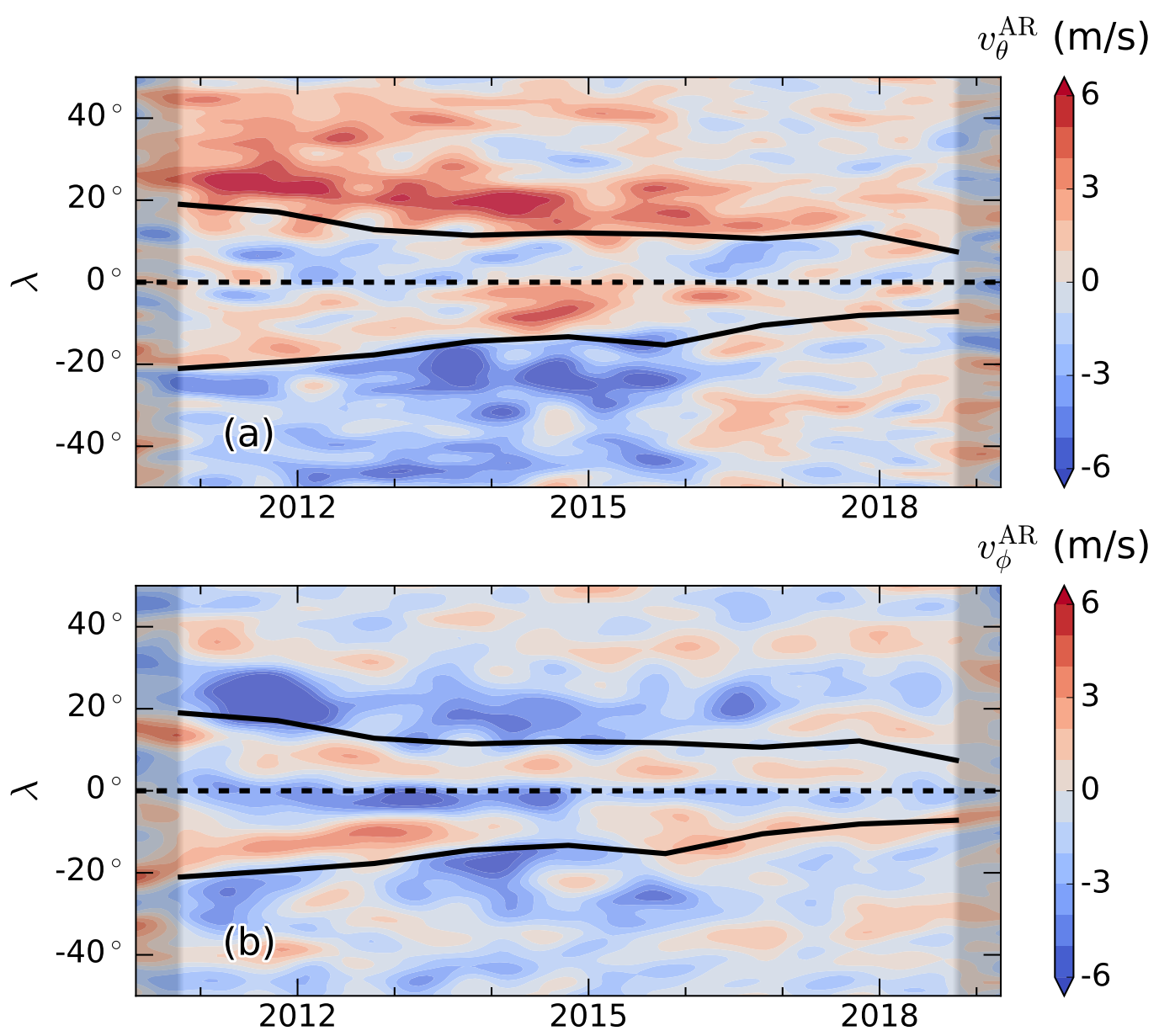

Figure 3.2: Longitudinal average of $v_{\theta}^{\mathrm{AR}}$ (panel $a$; positive values are southward) and $v_{\phi}^{\mathrm{AR}}$ (panel $b$; positive values are prograde), as a function of time and latitude. They were further smoothed in latitude with a Gaussian of FWHM of $3.6^{\circ}$ and in time with a Gaussian of FWHM of 6 months. The black lines show the mean active latitudes. The ticks on the horizontal axis indicate the beginning of each year. The shaded areas indicate the times when the edge effects become visible due to the one-year temporal smoothing of the background flows.

We track these active-region flow maps at the Carrington rate on a daily basis using noon as a reference, and remap them using the Plate Carree projection into the heliographic coordinate system. The spatial resolution is $0.6^{\circ}$ per pixel. Following the formulas in the appendix of Löptien et al. (2017), we convert the velocities from pixels per second to meters per second. Then we average the flows daily. These daily-averaged flows are denoted by $v_{j}^{\mathrm{AR}}(\theta, \phi, t)$, where $j \in\{\theta, \phi\}$ indicates the colatitudinal or the longitudinal direction. 


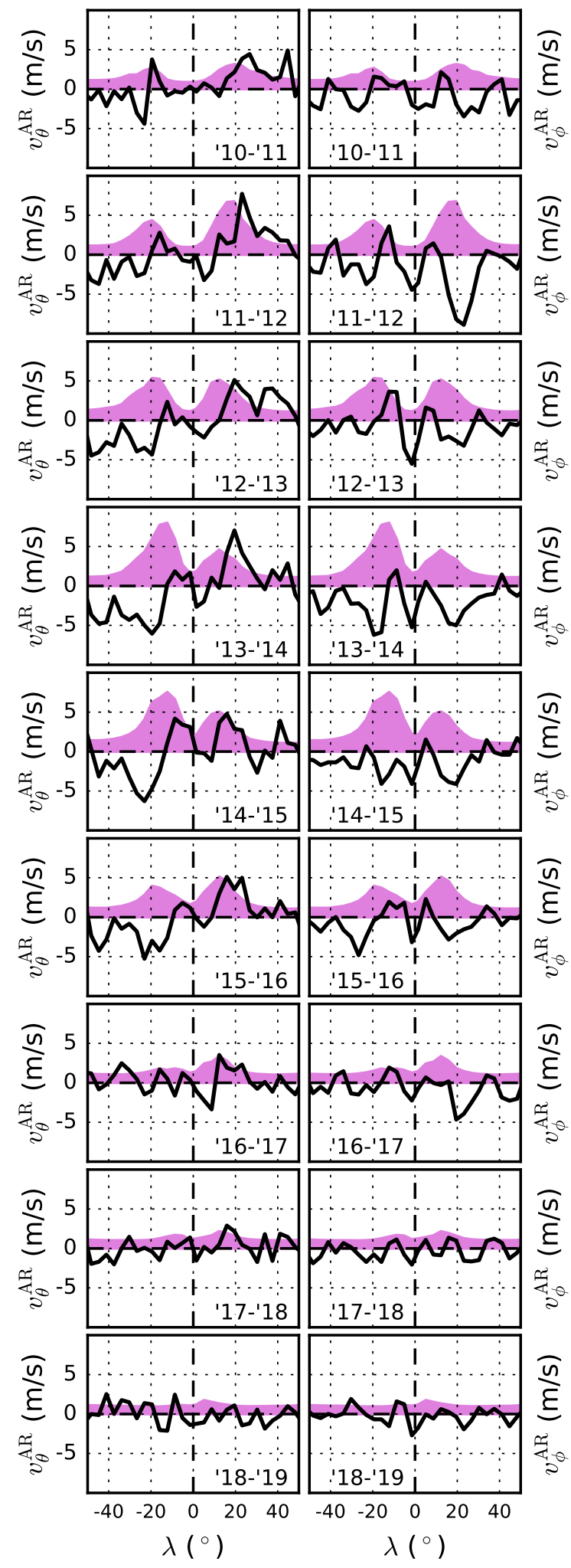

Figure 3.3: In black, longitudinally- and yearly-averaged $v_{\theta}^{\mathrm{AR}}$ and $v_{\phi}^{\mathrm{AR}}$, rebinned in latitude to obtain a point every $3.6^{\circ}$. The standard error of the mean in each binning interval is about $1 \mathrm{~m} / \mathrm{s}$ for $v_{\theta}^{\mathrm{AR}}$ and $0.4 \mathrm{~m} / \mathrm{s}$ for $v_{\phi}^{\mathrm{AR}}$, and is not shown here. The magenta filling indicates the distribution of $\left|B_{r}\right|$ (arbitrary unit), which was averaged and rebinned in the same way as the flows. 


\subsection{Temporal variation of active-region flows}

To examine the temporal and latitudinal evolution of the active-region flows, we average $v_{j}^{\mathrm{AR}}(\theta, \phi, t)$ in longitude within $\pm 15^{\circ}$ of the central meridian. Figure 3.2 shows the longitudinal averages of $v_{\theta}^{\mathrm{AR}}$ and $v_{\phi}^{\mathrm{AR}}$ as a function of time and latitude (denoted as $\lambda=90^{\circ}-\theta$ ). Figure 3.3 shows the yearly-averaged flows as a function of latitude, together with the magnetic activity.

Figure 3.2a shows an inflow pattern converging toward the mean latitude of activity in both hemispheres. The inflows are stronger and cover a greater latitudinal range on the poleward side of the active latitude. We note that, in Braun (2019) and Gottschling et al. (2021), the inflows are mostly symmetric with respect to the center of the active regions. This is because they use an ensemble averaging over active regions whose centers have been aligned with each other, while we use a longitudinal long-term averaging that makes the equatorward side of the inflows from both hemispheres cancel each other out.

In the left column of Fig. 3.3, the amplitude of the yearly-averaged $v_{\theta}^{\mathrm{AR}}$ reaches extrema of over $7 \mathrm{~m} / \mathrm{s}$ in the north in 2011-2012 and over $6 \mathrm{~m} / \mathrm{s}$ in the south in 20142015. Likewise, the solar magnetic activity peaks in 2011 in the northern hemisphere and in 2014 in the southern hemisphere. Therefore the amplitude of the inflows is clearly correlated with the strength of the solar activity. This amplitude is consistent with that predicted by the model of Spruit (2003) that interprets the inflows as a consequence of the enhanced cooling in the activity belt. The amplitude is also similar to that from, e.g., Gizon (2003), González Hernández et al. (2008), Komm et al. (2020), who isolated regions of magnetic activity and their surroundings in a way similar to what we do here.

Figure $3.2 \mathrm{~b}$ shows that the toroidal component is a retrograde flow (with respect to the background flow) on the poleward side of the active latitudes. The torsional oscillations, which are the time-varying part of the solar rotation, exhibit a shear flow around the active latitude, with a faster-rotating band on the equatorward side and a slower-rotating one on the poleward side. Therefore, the toroidal component seen here contributes to the torsional oscillations.

The right column of Fig. 3.3 shows that the amplitude of the toroidal component is also correlated with the amplitude of the solar magnetic activity. The retrograde flow reaches over $8 \mathrm{~m} / \mathrm{s}$ in the north in 2011-2012 and over $6 \mathrm{~m} / \mathrm{s}$ in the south in 2013-2014. This signal is consistent with the retrograde flow, surrounding active regions preferentially on the poleward side, that was found by Braun (2019). There is also a weaker prograde flow on the equatorward side of the activity belt. The presence of this signal is also consistent with the idea of a cyclonic circulation around active regions that derives from the model of Spruit (2003) and that was also described by Hindman et al. (2009). Braun (2019) and Gottschling et al. (2021) reported the presence of a prograde flow inside the leading polarity; the fact that the leading polarity lies at lower latitudes than the trailing one may also explain why a prograde flow is seen in our longitudinal and long-term average. 


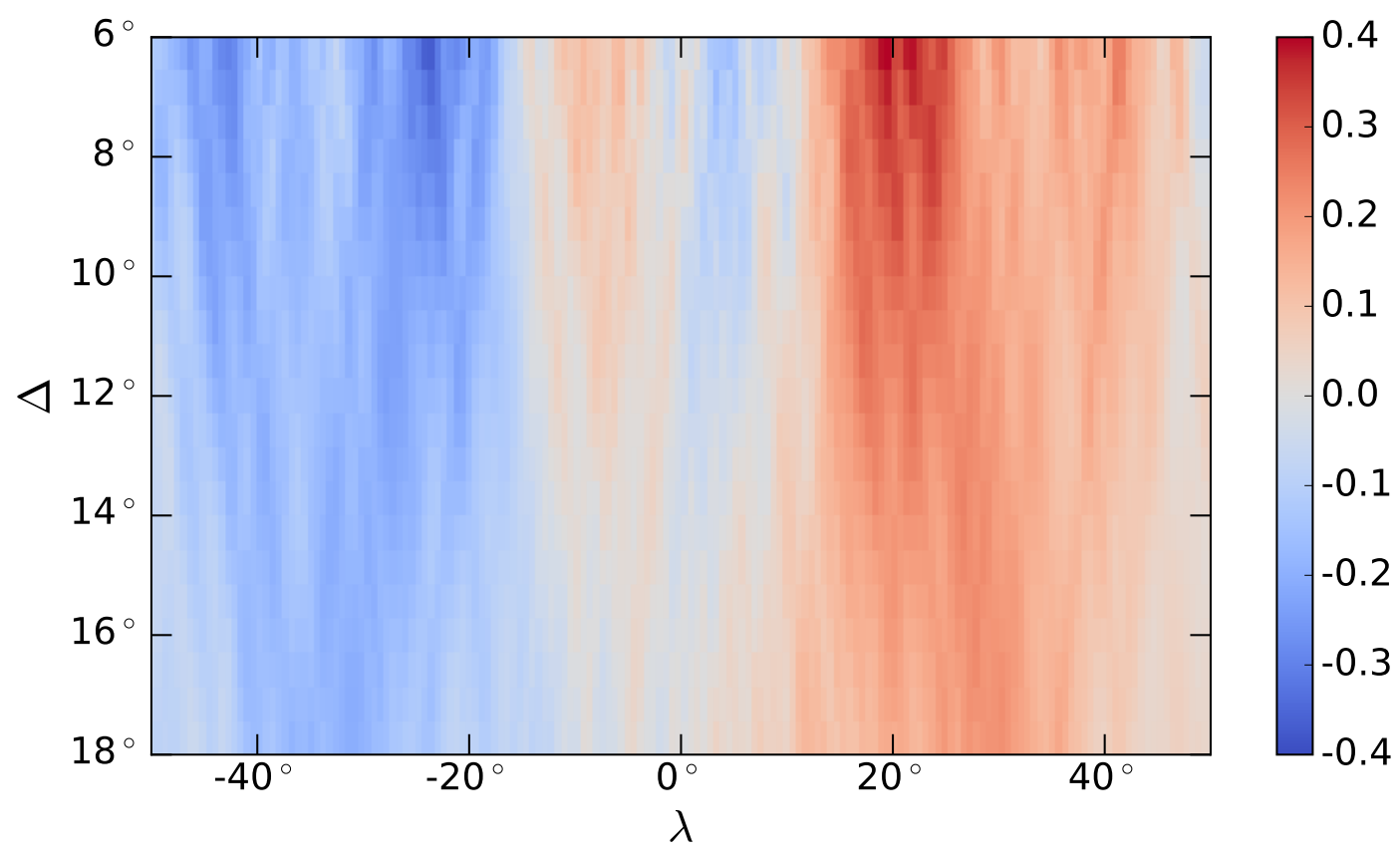

Figure 3.4: Forward travel-time perturbations $-\tau^{\mathrm{AR}}$, as a function of latitude and separation distance. They have been averaged in longitude around the central meridian and in time from January 2011 to December 2014.

\subsection{Modeled helioseismic travel-time perturbations}

\subsubsection{Computation of travel-time perturbations}

We compute the forward travel-time perturbations associated with the active-region flows to compare with the solar-cycle variations of the helioseismic measurements of the meridional circulation.

To this end, we first construct a 3D flow profile associated with the active-region flows at the surface. We neglect the contribution from the vertical flow to the travel times. For the colatitudinal and longitudinal components $u_{\theta}$ and $u_{\phi}$, we choose a constant profile with depth such that

$$
\begin{aligned}
& u_{\theta}(\boldsymbol{r}, t)=v_{\theta}^{\mathrm{AR}}(\theta, \phi, t), \\
& u_{\phi}(\boldsymbol{r}, t)=v_{\phi}^{\mathrm{AR}}(\theta, \phi, t),
\end{aligned}
$$

where $\boldsymbol{r}=(r, \theta, \phi)$. Although it would be more realistic to choose a flow profile where the amplitude decreases with depth, choosing a constant profile allows us to estimate an upper bound for the amplitude of the travel-time perturbations due to active-region flows.

In order to directly compare with Gizon et al. (2020), we use an arc-to-arc geometry, with the travel-time perturbations being computed between pairs of points placed on two opposite arcs. The paired arcs, each subtending an angle of $30^{\circ}$, are aligned in the northsouth direction as described by Liang et al. (2017). Let's denote $\psi$ the angle between a meridian and the ray path connecting the paired points on the $\operatorname{arcs},\left(\theta_{0}, \phi_{0}\right)$ the mid-point 

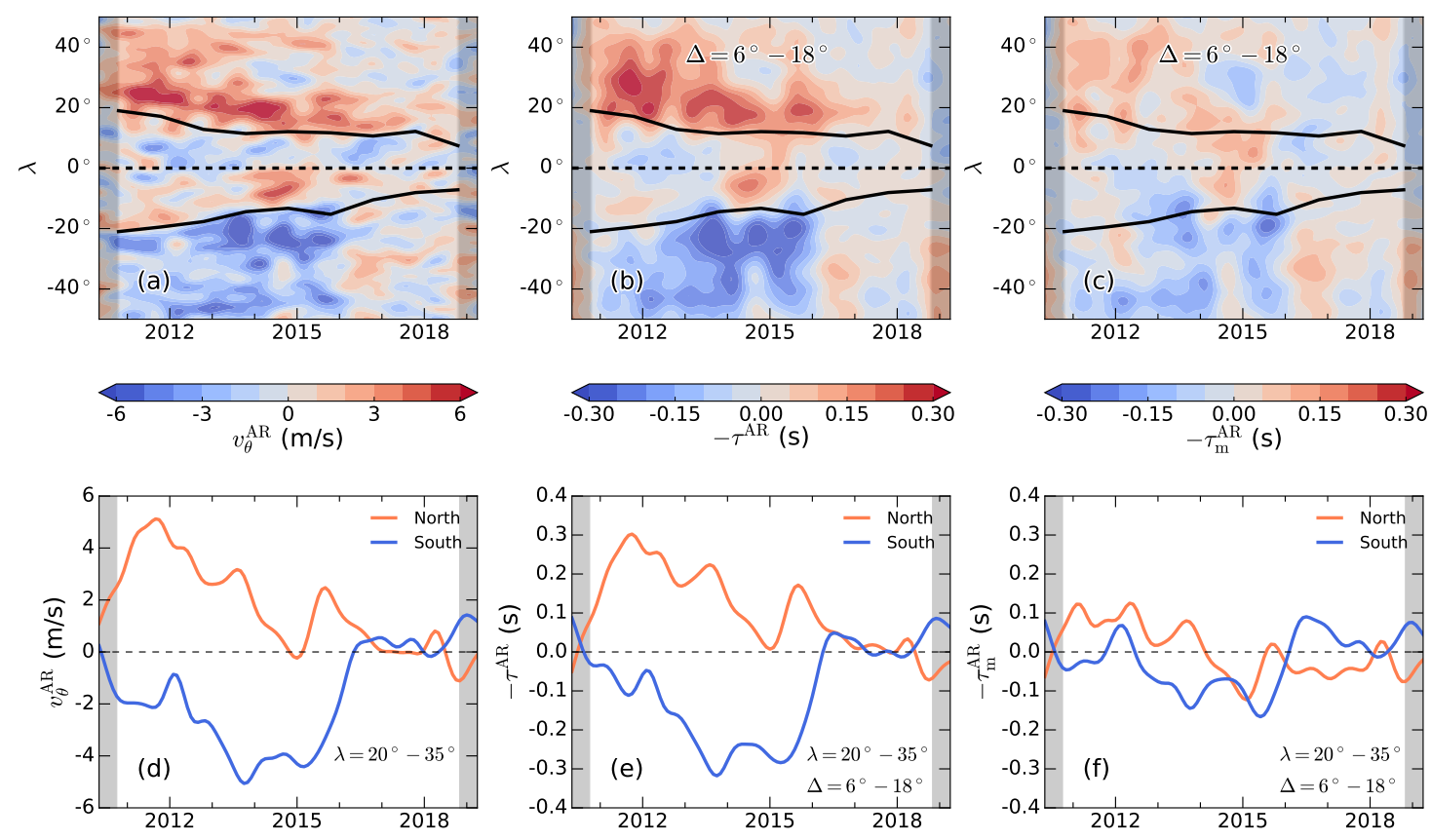

Figure 3.5: Panel a: Longitudinally-averaged active-region flows $v_{\theta}^{\mathrm{AR}}$ as plotted in Fig. 3.2, shown again here for convenience of comparison. Panel $b$ : Longitudinallyaveraged forward travel-time perturbations $-\tau^{\mathrm{AR}}$, further averaged over separation distances $6^{\circ}-18^{\circ}$. Panel $c$ : same as panel $b$, but for the travel-time perturbations with the magnetic mask applied, $-\tau_{\mathrm{m}}^{\mathrm{AR}}$ (discussed in Section 3.4.2). We applied the same smoothing as in Fig. 3.2. The black lines show the mean active latitudes. Panels $d-f$ : Averages of the top panels over latitudes $\lambda=20^{\circ}-35^{\circ}$ in each hemisphere. The typical errors in the flows over the latitudinal range of interest are about $0.6 \mathrm{~m} / \mathrm{s}$. For the travel-time perturbations, the errors are in the range of $0.002-0.008 \mathrm{~s}$, with the largest error for $\tau_{\mathrm{m}}^{\mathrm{AR}}$ during the peaks of activity. The errors are not shown here. In all panels, the shaded areas indicate the times when the edge effects become visible due to the one-year temporal smoothing of the background flows.

between the paired arcs, and $\Delta$ the separation distance between the paired points. The travel-time perturbation is thus defined as (e.g., Gizon et al. 2017, Fournier et al. 2018)

$$
\begin{aligned}
\tau\left(\theta_{0}, \phi_{0}, \Delta, \psi, t\right)=\int_{\odot} & \left(K_{\theta}\left(\boldsymbol{r} ; \theta_{0}, \phi_{0}, \Delta, \psi\right) u_{\theta}(\boldsymbol{r}, t)\right. \\
& \left.+K_{\phi}\left(\boldsymbol{r} ; \theta_{0}, \phi_{0}, \Delta, \psi\right) u_{\phi}(\boldsymbol{r}, t)\right) \mathrm{d} \boldsymbol{r}
\end{aligned}
$$

where the integral is taken over the whole volume of the Sun, and $K_{\theta}$ and $K_{\phi}$ are the colatitudinal and longitudinal components of the sensitivity kernel (see Appendix 3.8.3). Here, by convention, a northward flow perturbation corresponds to a positive travel-time perturbation for short separation distances.

The forward travel-time perturbation is averaged in the same way as in Gizon et al. (2020); that is,

$$
\tau^{\mathrm{AR}}\left(\theta_{0}, \Delta, t\right)=\left(N_{\phi_{0}} N_{\psi}\right)^{-1} \times \sum_{\phi_{0}, \psi} \tau\left(\theta_{0}, \phi_{0}, \Delta, \psi, t\right),
$$


where the sum over $\phi_{0}$ is taken within $\pm 15^{\circ}$, the sum over $\psi$ is taken within $\pm 15^{\circ}, N_{\phi_{0}}$ is the number of points used in the longitudinal average, and $N_{\psi}$ is the number of points on an arc for each distance.

Figure 3.4 shows the forward travel-time perturbations as a function of latitude and separation distance after we averaged over the active period from January 2011 to December 2014. Even though we chose a constant flow profile with depth, the forward travel-time perturbations could still change sign with increasing separation distance, for example, when the separation distance is larger than the spatial scale of active region flows. Our $\tau^{\mathrm{AR}}$ decreases with separation distance but does not change sign up to at least $\Delta=18^{\circ}$, which is similar to the modeled travel-time perturbations from Liang et al. (2018).

We show the longitudinally-averaged $v_{\theta}^{\mathrm{AR}}$ (as plotted in Fig. 3.2) in Fig. 3.5a, and the corresponding $\tau^{\mathrm{AR}}$ averaged over separation distances $6^{\circ}-18^{\circ}$ in Fig. 3.5b. We can clearly see the inflow pattern converging toward the active latitudes in Fig. 3.5b, with an amplitude that varies throughout the solar cycle as in panel Fig. 3.5a. When averaged over latitudes $20^{\circ}-35^{\circ}$, the amplitude of the inflows reaches $5 \mathrm{~m} / \mathrm{s}$ in 2011 in the north and $-5 \mathrm{~m} / \mathrm{s}$ in 2014 in the south (Fig. 3.5d). Similarly, the amplitude of $\tau^{\mathrm{AR}}$ reaches extrema of $0.3 \mathrm{~s}$ during these active periods (Fig. 3.5e). For comparison, we also computed $\tau^{\mathrm{AR}}$ using the radial flow profile from the shallow model LC2 described in Liang et al. (2018); in this case, the amplitude of $\tau^{\mathrm{AR}}$ reaches extrema of $0.15 \mathrm{~s}$.

\subsubsection{Effect of masking travel-time perturbations inside magnetic re- gions}

Gizon et al. (2020) excluded the travel-time perturbations measured within magnetic regions from the averages, as those measurements introduce a systematic error that resembles a divergent flow pattern (Liang and Chou 2015). In order to compare with their results, we apply the same masking as they did. The contour of the mask is shown as the thin black line in Fig 3.1. We rewrite the forward travel-time perturbation averaging with a weighting function $w$ that is equal to zero if the forward travel-time perturbation is excluded and one elsewhere. Equation 3.4 then becomes

$$
\begin{aligned}
\tau_{\mathrm{m}}^{\mathrm{AR}}\left(\theta_{0}, \Delta, t\right) & =\left(\sum_{\phi_{0}, \psi} w\left(\theta_{0}, \phi_{0}, \Delta, \psi, t\right)\right)^{-1} \times \\
& \sum_{\phi_{0}, \psi} w\left(\theta_{0}, \phi_{0}, \Delta, \psi, t\right) \tau\left(\theta_{0}, \phi_{0}, \Delta, \psi, t\right) .
\end{aligned}
$$

The third column of Fig. 3.5 shows the forward travel-time perturbations after the magnetic mask is applied, denoted by $\tau_{\mathrm{m}}^{\mathrm{AR}}$. These perturbations are associated with the flows located between the contour defining the magnetic mask and the one defining the background (see Fig. 3.1). $\tau_{\mathrm{m}}^{\mathrm{AR}}$ (Fig. 3.5c) resembles $\tau^{\mathrm{AR}}$ (Fig. 3.5b) but the amplitude of the inflow pattern is strongly reduced during solar maximum. Figure $3.5 \mathrm{f}$ shows that, when averaged over mid latitudes, the amplitude reaches a maximum in 2011-2012 in the north and in 2014-2015 in the south, that is three times smaller than that in Fig. 3.5e. We note that $\tau_{\mathrm{m}}^{\mathrm{AR}}$ changes sign around the same time as the background flows (see Fig. 3.11): 


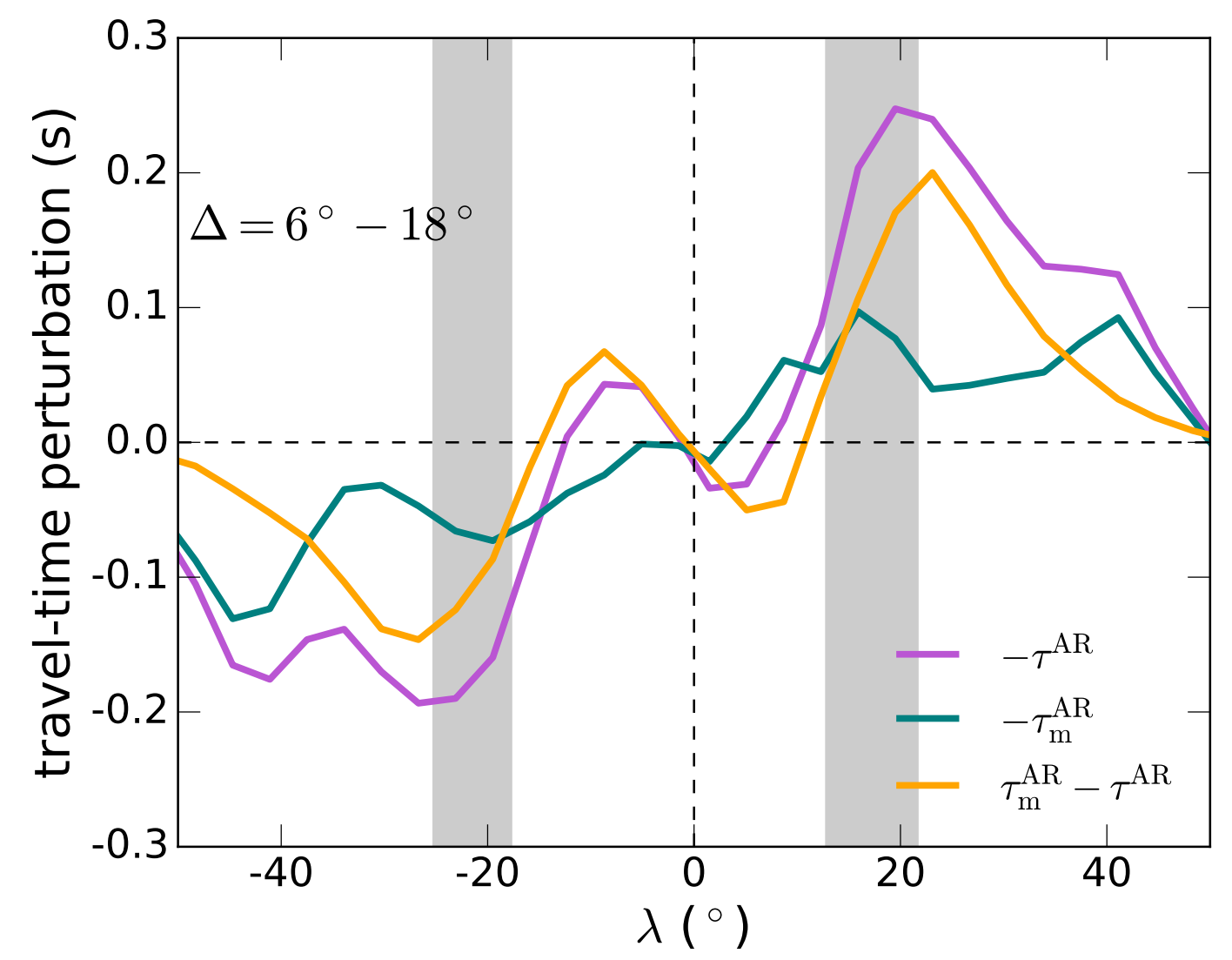

Figure 3.6: Longitudinally-averaged forward travel-time perturbations, further averaged from January 2011 to December 2014, i.e. during high magnetic activity. They have been rebinned in latitude to obtain a point every $3.6^{\circ}$. The typical standard error of the mean, computed in each binning interval, is about $0.003 \mathrm{~s}$, and is not shown here. The vertical gray shaded areas show the mean active latitudes.

the pattern is mostly equatorward during solar maximum and becomes poleward after 2016.

Figure 3.6 presents a comparison between $\tau^{\mathrm{AR}}$ and $\tau_{\mathrm{m}}^{\mathrm{AR}}$, averaged over four active years from January 2011 to December 2014. $\tau^{\mathrm{AR}}$ shows an inflow pattern with an amplitude reaching a maximum at about $20^{\circ}$. With the masking, the inflow pattern converging toward the active latitudes is nearly gone, and it rather looks like a flow converging toward the equator with an extremal amplitude of about $0.1 \mathrm{~s}$. The difference $\tau_{\mathrm{m}}^{\mathrm{AR}}-\tau^{\mathrm{AR}}$ presents a clear inflow pattern toward the active latitudes, comparable to $\tau^{\mathrm{AR}}$. Therefore, the masking of the magnetic regions removes most of the inflow pattern from the averaged travel-time perturbations.

We note that the masking of magnetic pixels implemented in Gizon et al. (2020) checks an area of $4 \times 4$ pixels around either of the foot points in the arc-to-arc geometry (Liang et al. 2017); if the field strength is greater than a threshold in this area, the paired points are excluded from the averages. We found that if only the nearest pixel to the foot point (instead of a $4 \times 4$-pixel area) is checked, the reduction of the inflow pattern 

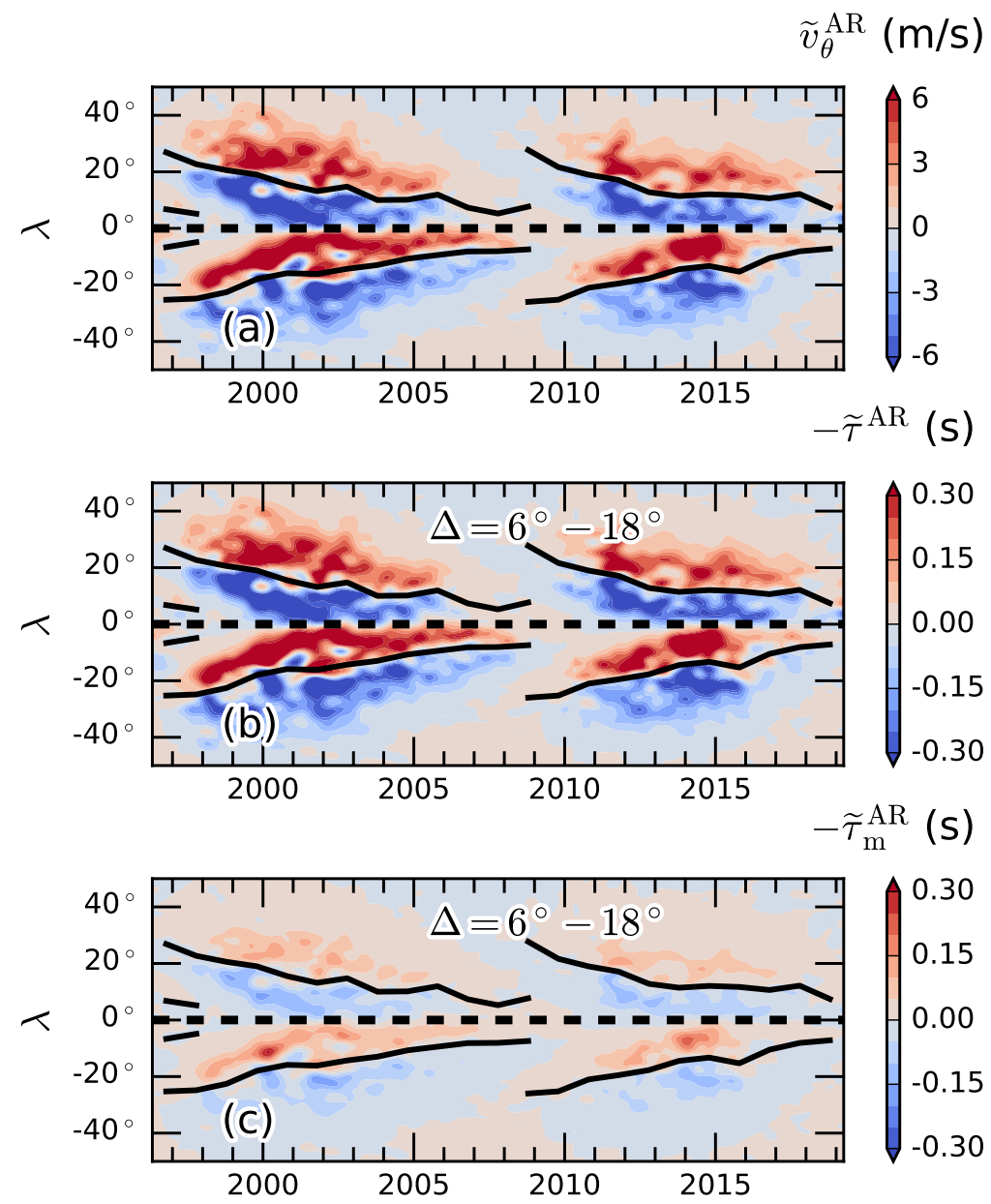

Figure 3.7: Modeled inflows $\widetilde{v}_{\theta}^{\mathrm{AR}}$ (panel a), and travel-time perturbations without magnetic mask $-\widetilde{\tau}^{\mathrm{AR}}$ (panel $b$ ) and with magnetic mask $-\widetilde{\tau}_{\mathrm{m}}^{\mathrm{AR}}$ (panel $c$ ) from May 1996 to April 2019. $\widetilde{\tau}^{\mathrm{AR}}$ and $\widetilde{\tau}_{\mathrm{m}}^{\mathrm{AR}}$ are derived from $\tau^{\mathrm{AR}}$ and $\tau_{\mathrm{m}}^{\mathrm{AR}}$ averaged over separation distances $6^{\circ}-18^{\circ}$. The same smoothing as in Fig. 3.2 is applied. The black lines show the mean active latitudes.

in the forward travel-time perturbations is not as strong as the aforementioned results.

\subsection{Extension to May 1996 - April 2019}

We aim to extend the analysis to cover the time period 1996-2019 in order to compare the results with Gizon et al. (2020). Although the LCT data are only available after May 2010 as they were computed from full-resolution HMI intensity images, we could use the magnetic field as a proxy for the active-region inflows from May 1996 to April 2010. The correlation between the magnetic field and the inflows is visible in Fig. 3.3: the greater the latitudinal gradient of the magnetic field strength, the greater the inflows. Several models already exist in the dynamo literature to generate artificial active-region inflows based on the observations of the magnetic field (e.g., De Rosa and Schrijver 2006, Cameron and Schüssler 2010, 2012). 

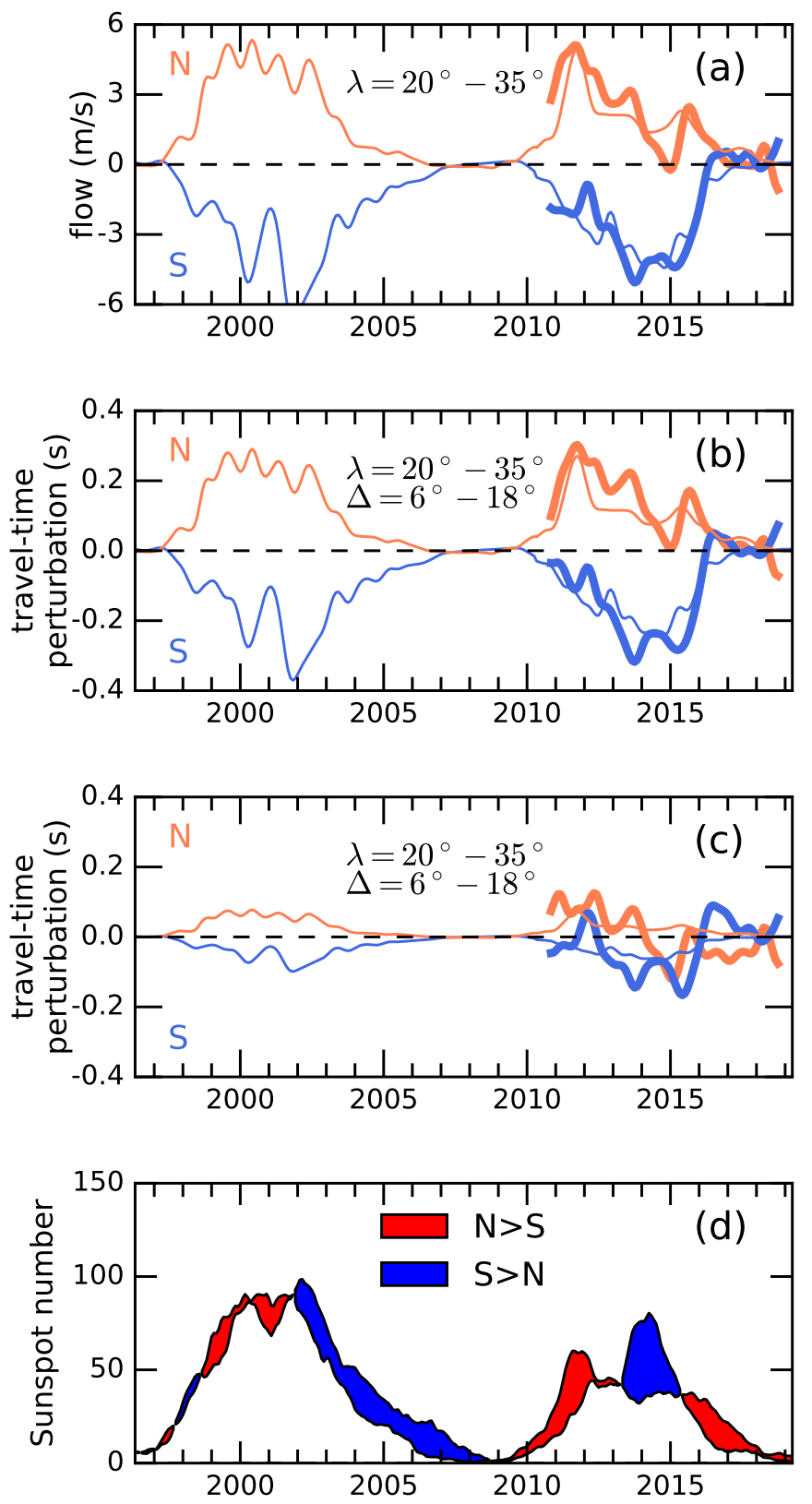

Figure 3.8: Panels $a-c$ : Averages of $\widetilde{v}_{\theta}^{\mathrm{AR}},-\widetilde{\tau}^{\mathrm{AR}}$, and $-\widetilde{\tau}_{\mathrm{m}}^{\mathrm{AR}}$ from Figs. 3.7a-c over latitudes $\lambda=20^{\circ}-35^{\circ}$ in each hemisphere (thin lines). For comparison, the corresponding $v_{\theta}^{\mathrm{AR}}$, $-\tau^{\mathrm{AR}}$, and $-\tau_{\mathrm{m}}^{\mathrm{AR}}$ from Figs. $3.5 \mathrm{~d}-\mathrm{f}$ are overplotted in thick lines; the data in the first and last six months are not shown because the edge effects become visible due to the one-year temporal smoothing of the background flows. Panel $d$ : 13-month running mean of the hemispheric monthly sunspot numbers. The red (respectively blue) filling indicates an excess of sunspot numbers in the northern (respectively southern) hemisphere. 
We minimize the cost function

$$
\left\|\left\langle v_{\theta}^{A R}\right\rangle-c_{0} \frac{\partial\left\langle\left|B_{r}\right|\right\rangle}{\partial \theta}\right\|^{2}
$$

to determine the constant of proportionality $c_{0}$. Here $\|\cdot\|$ denotes the $L_{2}$-norm and $\langle\cdot\rangle$ denotes the averaging in longitude within $\pm 15^{\circ}$ of the central meridian and the smoothing in latitude and time in the way described in Fig. 3.2. We use only the data from January 2011 to December 2014 and in the latitudinal range $20^{\circ}-35^{\circ}$, where the inflows are the strongest. We find $c_{0} \simeq 0.072 \mathrm{~m} \mathrm{~s}^{-1} \mathrm{G}^{-1}$.

We compute the modeled inflows $\widetilde{v}_{\theta}^{\mathrm{AR}}$ using the MDI magnetograms from May 1996 to April 2010 and the HMI magnetograms from May 2010 to April 2019. The line-ofsight magnetic field inferred from HMI data is smaller than that from MDI data (Liu et al. 2012); the scaling factor depends on the location on the disk and on the field strength. We determine this scaling factor using $\left|B_{r}\right|$ at latitudes $20^{\circ}-35^{\circ}$ and longitudes within $\pm 15^{\circ}$ of the central meridian, during the time period from May 2010 to April 2011 when both data sets are available. We find the scaling factor is about 0.64 which is then applied to the MDI data.

Finally, based on the similarity between the inflows and the travel-time perturbations shown in Fig. 3.5, we use a conversion constant to convert from the modeled flows $\widetilde{v}_{\theta}^{\mathrm{AR}}$ to modeled travel-time perturbations from May 1996 to April 2019. For an average over separation distances $6^{\circ}-18^{\circ}$, we find the conversion constant to be $-0.056 \mathrm{~s}^{2} / \mathrm{m}$ for the case without the magnetic mask $\widetilde{\tau}^{\mathrm{AR}}$ and $-0.014 \mathrm{~s}^{2} / \mathrm{m}$ for the case with the magnetic mask $\widetilde{\tau}_{\mathrm{m}}^{\mathrm{AR}}$.

Figure 3.7 shows the modeled inflows and the travel-time perturbations without and with magnetic mask as a function of time and latitude, over Cycle 23 (1996-2008) and Cycle 24 (2008-2019). All the panels exhibit the inflow pattern with the flows generally converging toward the mean active latitudes, which is similar to that in Fig. 3.5. The amplitude of the models is comparable to that of the observations for both the inflows and the travel-time perturbations on the poleward side of the activity belt. However, on the equatorward side, the amplitude of the models is greater because we determined the proportionality constant $c_{0}$ using only the latitudes $20^{\circ}-35^{\circ}$.

Figure 3.8 shows how the models compare with the observations when averaged over latitudes $20^{\circ}-35^{\circ} . \widetilde{v}_{\theta}^{\mathrm{AR}}$ (Fig. 3.8a) and $\widetilde{\tau}_{\theta}^{\mathrm{AR}}$ (Fig. 3.8b) generally match $v_{\theta}^{\mathrm{AR}}$ and $\tau^{\mathrm{AR}}$ well in both hemispheres, especially during the peaks of magnetic activity. When the magnetic mask is applied (Fig. 3.8c), the model $\widetilde{\tau}_{\mathrm{m}}^{\mathrm{AR}}$ retrieves the correct order of magnitude but does not fully capture $\tau_{\mathrm{m}}^{\mathrm{AR}}$. Furthermore, we note that the amplitude of the models is greater during the Cycle 23 solar maximum than that during the Cycle 24 solar maximum, which is consistent with the sunspot number presented in Fig. 3.8d.

\subsection{Comparison with helioseismic measurements}

We compare in Fig. 3.9 our forward travel-time perturbations with the measurements from Gizon et al. (2020) spanning Cycles 23 and 24. Gizon et al. (2020) used the data from SOHO/MDI and GONG for the periods from May 1996 to April 2003 and from May 2003 to April 2019, respectively; the travel times associated with the magnetic pixels 


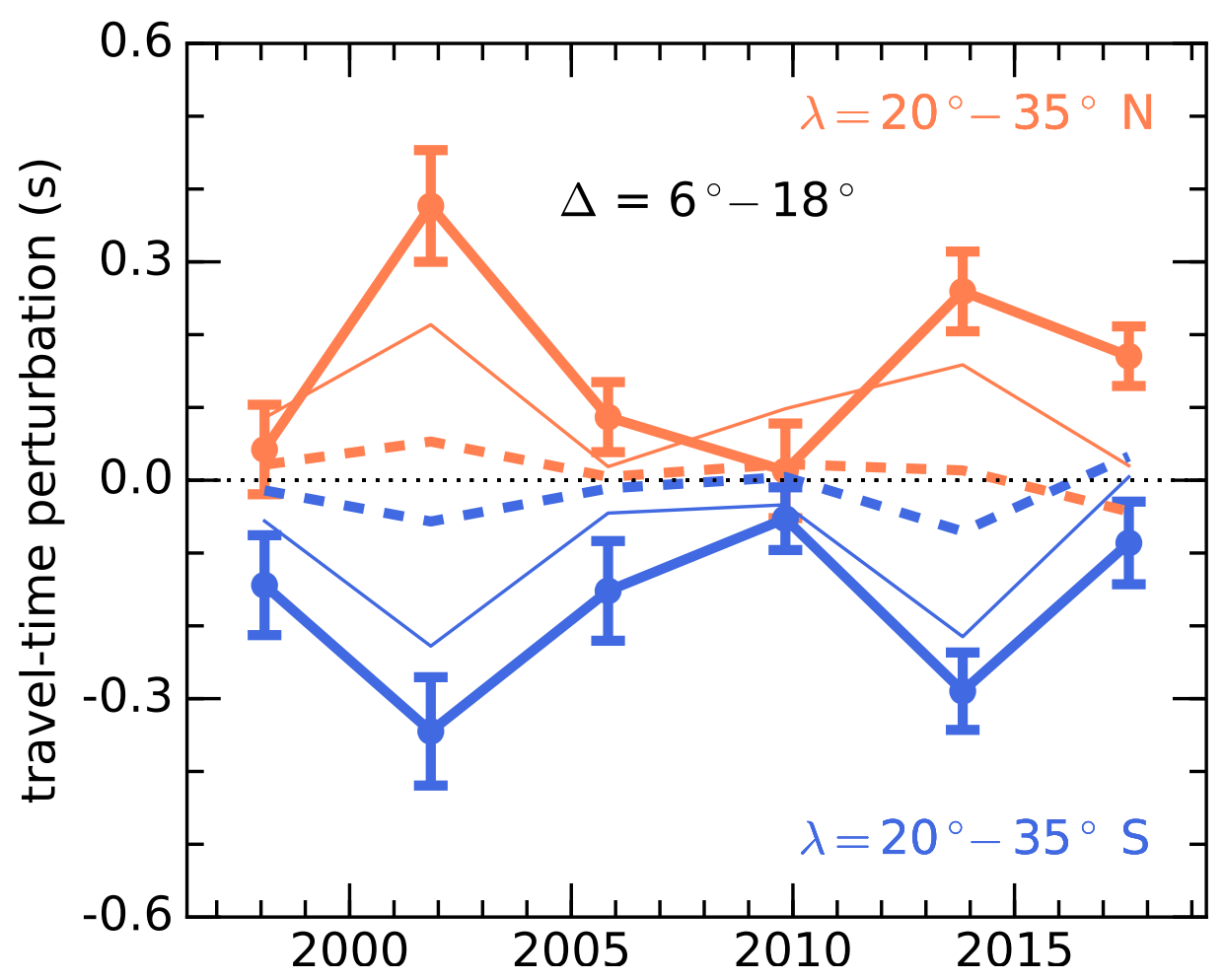

Figure 3.9: Comparison between the north-south travel-time perturbations as measured by Gizon et al. (2020) (thick solid lines) and the forward travel-time perturbations without (thin solid line) and with (thick dashed line) the magnetic mask. The measurements were averaged over the latitudes $\lambda=20^{\circ}-35^{\circ}$ in each hemisphere, over separation distances $\Delta=6^{\circ}-18^{\circ}$, and over consecutive 4-year time intervals. The average over the quiet-Sun period January 2007 to April 2009 was subtracted from the measurements, and the sign was reversed for the purpose of comparison. The corresponding $-\widetilde{\tau}^{\mathrm{AR}}$ and $-\widetilde{\tau}_{\mathrm{m}}^{\mathrm{AR}}$ are used from May 1996 to April 2010, and $-\tau^{\mathrm{AR}}$ and $-\tau_{\mathrm{m}}^{\mathrm{AR}}$ from May 2010 to April 2019. For the measurements, the error bars represent the standard error of the mean computed over the latitudinal range of interest. For the forward travel-time perturbations, the standard error of the mean is in the range of $0.001-0.006 \mathrm{~s}$ and is not shown here.

were excluded in their measurements. For the forward travel-time perturbations, we used $\widetilde{\tau}^{\mathrm{AR}}$ and $\widetilde{\tau}_{\mathrm{m}}^{\mathrm{AR}}$ for the period from May 1996 to April 2010, and $\tau^{\mathrm{AR}}$ and $\tau_{\mathrm{m}}^{\mathrm{AR}}$ for the period from May 2010 to April 2019.

The measurements of Gizon et al. (2020) exhibit a modulation in amplitude over the solar cycles that is correlated with the magnetic activity. The $\tau^{\mathrm{AR}}$ and $\widetilde{\tau}^{\mathrm{AR}}$ also exhibit this modulation, albeit with a weaker amplitude. We remind the reader that we used a constant profile with depth for the active-region flow model to place an upper limit; that is, the magnitude of $\tau^{\mathrm{AR}}$ and $\widetilde{\tau}^{\mathrm{AR}}$ is overestimated. When the masking is taken into account, the magnitude of $\tau_{\mathrm{m}}^{\mathrm{AR}}$ and $\widetilde{\tau}_{\mathrm{m}}^{\mathrm{AR}}$ differs greatly from the measurements. These results suggest that the true travel-time perturbations caused by the inflows do not fully explain the solar-cycle variations in the travel-time measurements. 


\subsection{Summary and discussion}

We used LCT flow maps over nine years during Cycle 24. We removed large-scale background flows and extracted the flows associated with active regions. We averaged these flows in longitude to study their latitudinal profile and time evolution. The latitudinal flow exhibits an inflow pattern converging toward the active latitudes with a maximal amplitude of $7 \mathrm{~m} / \mathrm{s}$ (yearly average) on the poleward side of the activity belt during solar maximum, which is about $50 \%$ of the global meridional flow at the surface. This amplitude is similar to that found by Gizon (2003), González Hernández et al. (2008), Komm et al. (2020), although a direct comparison with their results is not possible because they used different methods. The longitudinal flow exhibits a retrograde component on the poleward side of the activity belt, with an amplitude reaching $8 \mathrm{~m} / \mathrm{s}$, namely of the same order as the torsional oscillations. The presence and the structure of these flows are correlated with the strength and the distribution of the magnetic activity. The flows related to the surface magnetic activity thus strongly contribute to shaping the large-scale flows.

We computed the corresponding forward helioseismic travel-time perturbations using 3D sensitivity kernels and using an arc-to-arc geometry in the north-south direction. We assumed constant inflows with depth to place an upper limit on the contribution of inflows to helioseismic measurements of the meridional circulation. For separation distances $\Delta=$ $6^{\circ}-18^{\circ}$, the extrema of the averaged forward travel-time perturbations are $\pm 0.3 \mathrm{~s}$ during the peaks of solar activity. We also averaged the forward travel-time perturbations with the masking of magnetic regions as in Gizon et al. (2020), and found that the masking significantly reduced the amplitude of the travel-time perturbations, leading to extrema of about $\pm 0.1 \mathrm{~s}$ during solar maximum.

We extended the active-region flows and the forward travel-time perturbations to cover two solar cycles, from May 1996 to April 2019, using a model based on the latitudinal gradient of the magnetic field strength. We assumed that the travel-time perturbations are roughly proportional to the flows. This simple model reproduces the inflow patterns throughout the solar cycle and allows us to compare our results with the measurements of Gizon et al. (2020) over two solar cycles. We found that the near-surface active-region flows do not explain in full the solar-cycle variations seen in the measurements.

We note that the background flows, defined as the flows that are far from active regions, contain not only systematics but also true flows in the quiet regions, if any. We see that they exhibit a large-scale modulation throughout the solar cycle that seems correlated with the magnetic activity (Appendix 3.8.2). Since the LCT data were filtered to remove only the periods of $24 \mathrm{hr}, 1 \mathrm{yr}$, and the mean of the time series (Löptien et al. 2017, Gottschling et al. 2021), long-term variations of the global-scale meridional flow could still remain in the background flows. However, we cannot exclude that there exist systematics that vary with the solar cycle. Separating the true global-scale flows from the systematics in the LCT data is beyond the scope of this paper.

González Hernández et al. (2008) used ring-diagram analysis to infer the subsurface meridional flow, and found that the inflows persist even after the flows surrounding the active regions were excluded. They attributed it to the fact that their masking may not remove the weaker magnetic regions and the diffuse field, but they also did not exclude the possibility that inflows may exist in quiet regions. Similarly, Komm et al. (2020) studied the time variations of subsurface flows for quiet and active regions, separately, 
over the past two solar cycles and found a solar-cycle modulation in the quiet-Sun flows.

Hathaway and Rightmire (2010) found that the meridional flow is stronger during solar minimum, although they did not disentangle the active-region flows from the global circulation. Lin and Chou (2018) assumed that the meridional flows are simply a linear combination of the local inflows and global-scale meridional flows and found a similar result. A number of other studies, in which the authors subtracted a time-averaged meridional flow profile, observed residuals during solar minima (e.g., Hathaway and Rightmire 2011, Komm et al. 2015, Getling et al. 2021); however, the patterns of these residuals differ from one another, depending on the time periods used to compute the reference. To avoid this dependence, González Hernández et al. (2010) subtracted a low-order polynomial fit and also found meridional flow residuals. These studies support the conclusion of our paper that there may be a time-varying component of the meridional flow unrelated to active regions.

We note finally that other phenomena related to the surface magnetic activity can contribute to the temporal variation of the travel-time perturbations measured by Gizon et al. (2020). In particular, the Woodard effect (Woodard 1997) can add systematics via the localized absorption of acoustic waves by sunspots. This effect has not been taken into account in our study.

\section{Acknowledgements}

P.-L. P. is part of the International Max Planck Research School. We thank B. Löptien for kindly accepting to provide the LCT data series. The computational resources were provided by the German Data Center for SDO through grant 50OL1701 from the German Aerospace Center (DLR). The sunspot numbers are from WDC-SILSO, Royal Observatory of Belgium, Brussels.

\subsection{Appendix}

\subsubsection{Definition of contour}

To determine the values of the contour parameter $b$ defined in Section 3.2.2.1, we first compute the ensemble average of active regions in a way similar to Braun (2019) and Gottschling et al. (2021). A brief description of the procedure is as follows.

We track the HMI magnetograms at the Carrington rate, remap them using the Plate Carree projection into the heliographic coordinate system, and compute daily averages of unsigned magnetic flux density maps. We smooth these maps by a 2D Gaussian of full width at half maximum $10^{\circ}$ (heliographic degrees). We follow the algorithm of Braun (2019) to detect the peaks (pixels of value higher than that of any of the eight neighboring pixels), sort them from highest to lowest flux density, and then retain only those that are further than $20^{\circ}$ from all peaks of higher flux density. This procedure ensures that all the selected active regions are clearly separated from each other. Each active region has an associated total unsigned magnetic flux computed over a box, spanning $20^{\circ}$ in longitude and $10^{\circ}$ in latitude around the active region. We retain the active regions with a total unsigned magnetic flux above $10^{21} \mathrm{Mx}$. 


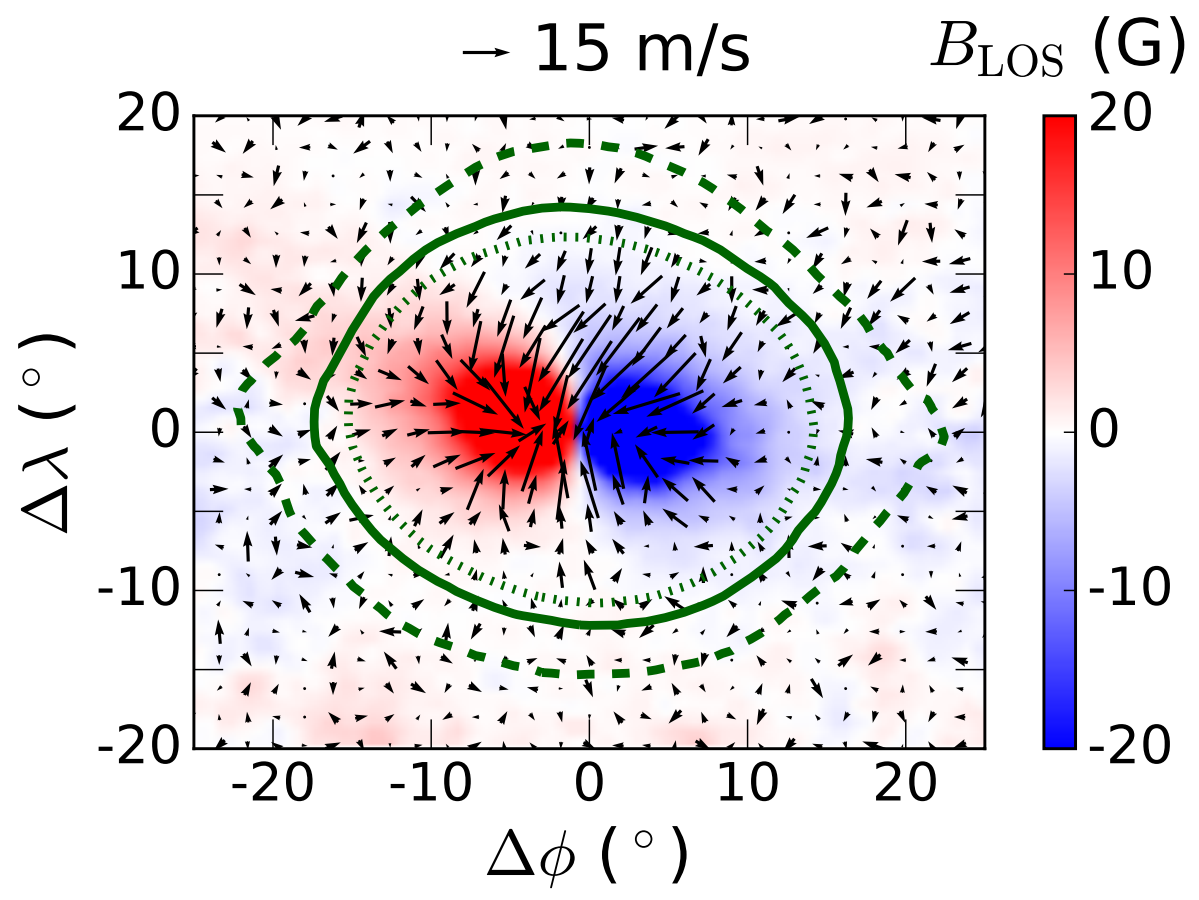

Figure 3.10: Average active-region flows. $\Delta \phi$ and $\Delta \lambda$ are the longitude and latitude with respect to the center of the active region. The flows close to the center of each polarity have been masked out before averaging because LCT flows in highly-magnetic regions are not reliable (Löptien et al. 2017). We superimpose the average contours for the following values of $b: 4 \mathrm{G}, 3.5 \mathrm{G}$, and $3 \mathrm{G}$ (dotted, solid, and dashed green lines, respectively).

We align the selected active regions with respect to their center of mass. The center of mass is computed as the average of the center of mass of the pixels with positive $B_{r}$ and that of the pixels with negative $B_{r}$, both weighted by the smoothed and daily averaged unsigned flux density. To limit the impact of noise, we do not consider pixels with $\left|B_{r}\right|$ less than $50 \mathrm{G}$ in the computation of the center of mass and of the magnetic flux. If an active region is in the southern hemisphere, we flip it in the north-south direction and the sign of $B_{r}$ and $v_{\theta}^{\mathrm{AR}}$, so that it can be averaged together with the active regions in the northern hemisphere.

Figure 3.10 shows the average active-region flows using the $v_{\theta}^{\mathrm{AR}}$ and $v_{\phi}^{\mathrm{AR}}$ from Section 3.2.3. They consist of inflows toward the center of the active region, mainly toward the trailing polarity. They extend on average up to $10^{\circ}$ away from the center of each polarity, in both the latitudinal and the longitudinal directions. This is consistent with the results of, e.g., Löptien et al. (2017), Braun (2019) and Gottschling et al. (2021).

We determine the contours for each individual active region with various values for the magnetic threshold $b$. For each value of $b$, we draw in Fig. 3.10 the line for which the pixels are inside of $90 \%$ of the contours. The contours for $b=4 \mathrm{G}$ and $3.5 \mathrm{G}$ are similar, with the later covering up to $12^{\circ}$ away from the active region in the latitudinal direction, that is, further than the inflows. However, using $b=4 \mathrm{G}$ results in yearly- and 
longitudinally-averaged flows up to $2-3 \mathrm{~m} / \mathrm{s}$ weaker than with $b=3.5 \mathrm{G}$. On the other hand, the contour with $b=3 \mathrm{G}$ covers up to about $15^{\circ}$ away from the active region, which seems more than enough to encompass all active-region flows. However with this parameter value, the background flows contains no data for more than a year at mid latitudes around 2011 in the north and around 2014 in the south. A smoothing in time with a Gaussian of FWHM of one year is therefore not possible. Increasing significantly the FWHM is also not possible because of the time scale of variation of the background flows. As a result, we choose $b=3.5 \mathrm{G}$ as the best compromise to consider as much active-region flows as possible and still have a reliable background estimation.

\subsubsection{Temporal variation of the background flows}

We track, remap and average the background flows in the same way as the active-region flows in order to study their temporal variation. The results are presented in Figure 3.11. After subtraction of the activity-related flows from the LCT maps, a large-scale modulation of the meridional component is still present, with a peak-to-peak amplitude of about $12 \mathrm{~m} / \mathrm{s}$ on average over mid latitudes (panel $c$ ). This trend is roughly antisymmetric with respect to the equator, and resembles an equatorward flow in the first half of the time series, then a poleward flow in the second half toward solar minimum (panel $a$ ). This is consistent with Gottschling et al. (2021). Although these flows contain systematics, this trend seems to indicate that the global-scale meridional flows become stronger when approaching the solar minimum, as was previously reported by, e.g., Hathaway and Rightmire (2010), González Hernández et al. (2010), Komm et al. (2015), Lin and Chou (2018), Gizon et al. (2020), and Getling et al. (2021).

The toroidal component also shows a modulation that is roughly symmetric with respect to the equator. It resembles a prograde flow with respect to the mean rotation rate in the first half of the time series, then a retrograde flow in the second half (panel $b$ ). The amplitude varies between -3 and $+5 \mathrm{~m} / \mathrm{s}$ (panel $d$ ). The prograde pattern during the first half migrates toward the equator along with the activity belts, which suggests that this component of the background flows might contain not only systematics but also real flows in the quiet Sun.

\subsubsection{D Born sensitivity kernels}

The computation of the 3D Born sensitivity kernels is based on the approaches from Gizon et al. (2017), Fournier et al. (2018). The wave field $\psi(\boldsymbol{r}, \omega)$ is solution of a scalar wave equation in the frequency domain

$$
-\left(\omega^{2}+2 \mathrm{i} \omega \gamma\right) \psi-2 \mathrm{i} \omega \boldsymbol{u} \cdot \nabla \psi-c \nabla \cdot\left(\frac{1}{\rho} \nabla(\rho c \psi)\right)=s(\boldsymbol{r}, \omega),
$$

where $\rho$ is the density, $c$ the sound speed, $\gamma$ the attenuation and $s$ a stochastic source term describing wave excitation. The wave field is related to the 3D wave displacement $\xi$ through $\psi(\boldsymbol{r}, \omega)=c(\boldsymbol{r}) \nabla \cdot \boldsymbol{\xi}(\boldsymbol{r}, \omega)$.

The flow kernels $\mathbf{K}=\left(K_{r}, K_{\theta}, K_{\phi}\right)$ can be computed from the knowledge of the Green's 
3 Contribution of flows around active regions to the north-south helioseismic measurements
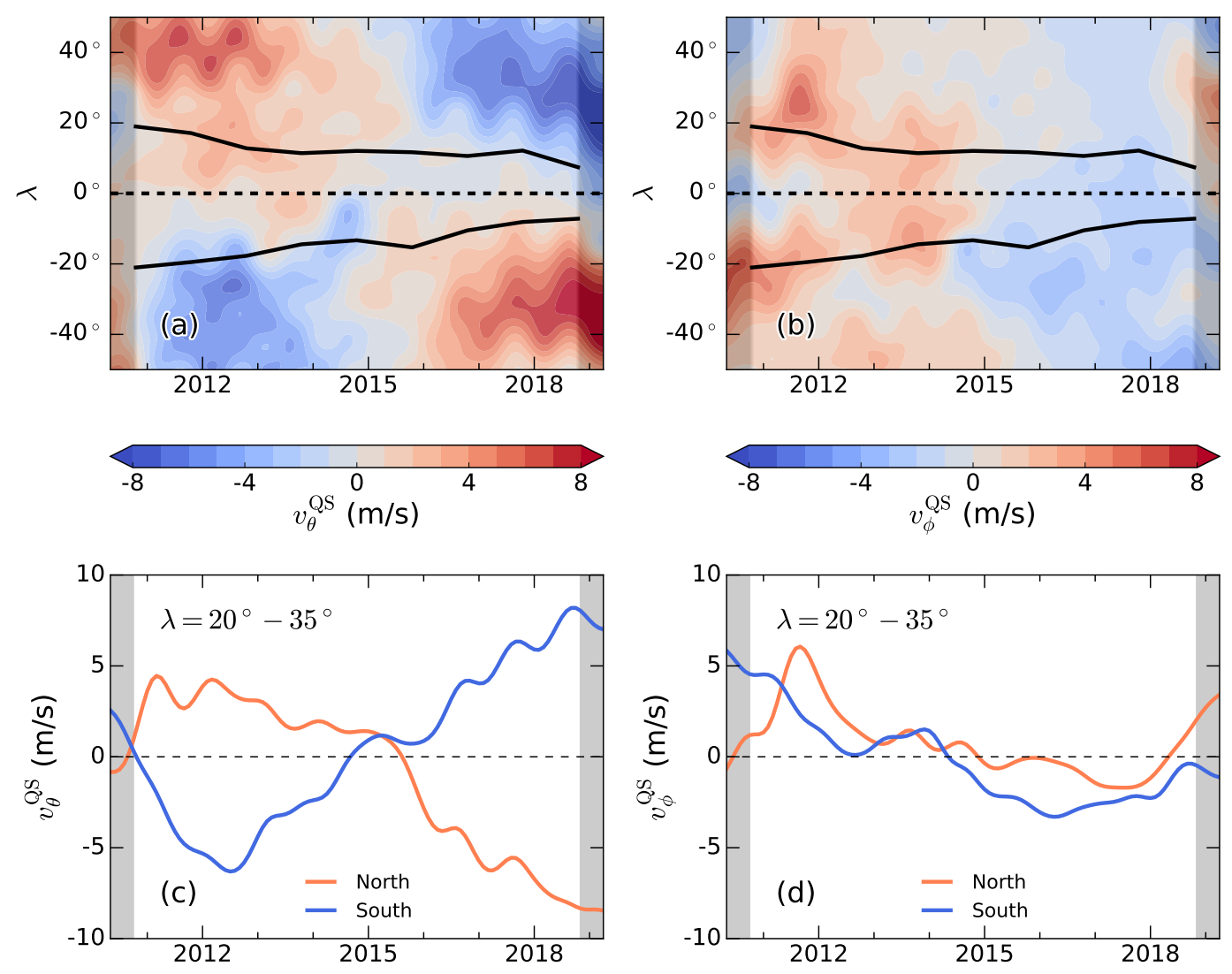

Figure 3.11: Longitudinally-averaged background flows $v_{\theta}^{\mathrm{QS}}$ (panel $a$; positive values are southward) and $v_{\phi}^{\mathrm{QS}}$ (panel $b$; positive values are prograde). We smoothed in latitude with a Gaussian of FWHM of $3.6^{\circ}$ and in time with a Gaussian of FWHM of 6 months. Panels $c-d$ : Average over latitudes $\lambda=20^{\circ}-35^{\circ}$ in each hemisphere; the typical standard errors of the mean, computed over the latitudinal interval, are about $0.06 \mathrm{~m} / \mathrm{s}$ for $v_{\theta}^{\mathrm{QS}}$ and about $0.03 \mathrm{~m} / \mathrm{s}$ for $v_{\phi}^{\mathrm{QS}}$, and are not shown here. In all panels, the shaded areas indicate the times when the edge effects become visible due to the one-year temporal smoothing of the flows.

function solution of Eq. (3.7) with a Dirac on the right hand side:

$$
\begin{gathered}
\mathbf{K}\left(\boldsymbol{r} ; \boldsymbol{r}_{1}, \boldsymbol{r}_{2}\right)=2 \mathrm{i} \rho(r) \int_{-\infty}^{\infty} \omega W^{*}\left(\boldsymbol{r}_{1}, \boldsymbol{r}_{2}, \omega\right)\left[G\left(\boldsymbol{r}_{2} ; \boldsymbol{r}, \omega\right) \nabla C\left(\boldsymbol{r}_{1} ; \boldsymbol{r}, \omega\right)\right. \\
\left.-G^{*}\left(\boldsymbol{r}_{1} ; \boldsymbol{r}, \omega\right) \nabla C^{*}\left(\boldsymbol{r}_{2} ; \boldsymbol{r}, \omega\right)\right] \mathrm{d} \omega,
\end{gathered}
$$

where $\boldsymbol{r}_{1}=\left(R, \theta_{1}, \phi_{1}\right)$ and $\boldsymbol{r}_{2}=\left(R, \theta_{2}, \phi_{2}\right)$ are two observation points, $R$ is the observation radius, $C$ is the cross-covariance, $W$ is a weighting function in order to relate the travel-time perturbation to changes in the cross-covariance, and $\nabla$ is the gradient operator with respect to $\boldsymbol{r}$. As in Gizon et al. (2017), we assume energy equipartition so that the cross-covariance is related to the imaginary part of the Green's function. For the sake of simplicity, we now drop the $\omega$ in the notation of the cross-covariance and of the Green's function. In a spherically symmetric background, the Green's function depends only on 
the angular distance between source and receiver and can be obtained from its Legendre coefficients (Fournier et al. 2018):

$$
G\left(r, \theta, \phi ; \boldsymbol{r}_{i}\right)=\frac{1}{\sqrt{2 \pi}} \sum_{\ell} G_{\ell}(r ; R) P_{\ell}\left(\cos \gamma_{i}\right),
$$

where $i \in\{1,2\}, P_{\ell}$ is the Legendre polynomial of order $\ell$,

$$
\cos \gamma_{i}=\cos \theta \cos \theta_{i}+\sin \theta \sin \theta_{i} \cos \left(\phi-\phi_{i}\right),
$$

and $G_{\ell}(r ; R)$ is solution of

$$
-\left(\omega^{2}+2 \mathrm{i} \omega \gamma\right) G_{\ell}-c \frac{d}{d r}\left(\frac{1}{\rho} \frac{d}{d r}\left(\rho c G_{\ell}\right)\right)+\frac{\ell(\ell+1) c^{2}}{r^{2}} G_{\ell}=\delta(r-R) .
$$

We keep the values of $\ell$ up to 300 .

Inserting Eq. (3.9) into Eq. (3.8), the kernel for the radial flow is given by

$$
\begin{aligned}
K_{r}\left(\boldsymbol{r} ; \boldsymbol{r}_{1}, \boldsymbol{r}_{2}\right)=\frac{2 \rho(r)}{\pi} \sum_{\ell \ell^{\prime}}[ & -f_{\ell \ell^{\prime}}^{r}(r) P_{\ell^{\prime}}\left(\cos \gamma_{1}\right) P_{\ell}\left(\cos \gamma_{2}\right) \\
& \left.+g_{\ell \ell^{\prime}}^{r}(r) P_{\ell}\left(\cos \gamma_{1}\right) P_{\ell^{\prime}}\left(\cos \gamma_{2}\right)\right],
\end{aligned}
$$

where

$$
\begin{aligned}
& f_{\ell \ell^{\prime}}^{r}(r)=\int_{0}^{\infty} \omega \operatorname{Im}\left[W^{*}\left(\boldsymbol{r}_{1}, \boldsymbol{r}_{2}, \omega\right) G_{\ell}(r ; R) \partial_{r} C_{\ell^{\prime}}(r ; R)\right] \mathrm{d} \omega, \\
& g_{\ell \ell^{\prime}}^{r}(r)=\int_{0}^{\infty} \omega \operatorname{Im}\left[W^{*}\left(\boldsymbol{r}_{1}, \boldsymbol{r}_{2}, \omega\right) G_{\ell}^{*}(r ; R) \partial_{r} C_{\ell^{\prime}}^{*}(r ; R)\right] \mathrm{d} \omega .
\end{aligned}
$$

Similarly, denoting

$$
\begin{aligned}
& f_{\ell \ell^{\prime}}^{j}(r)=\int_{0}^{\infty} \omega \operatorname{Im}\left[W^{*}\left(\boldsymbol{r}_{1}, \boldsymbol{r}_{2}, \omega\right) G_{\ell}(r ; R) C_{\ell^{\prime}}(r ; R)\right] \mathrm{d} \omega, \\
& g_{\ell \ell^{\prime}}^{j}(r)=\int_{0}^{\infty} \omega \operatorname{Im}\left[W^{*}\left(\boldsymbol{r}_{1}, \boldsymbol{r}_{2}, \omega\right) G_{\ell}^{*}(r ; R) C_{\ell^{\prime}}^{*}(r ; R)\right] \mathrm{d} \omega,
\end{aligned}
$$

where $j \in\{\theta, \phi\}$, the kernels for the horizontal flow components are given by

$$
\begin{aligned}
K_{j}\left(\boldsymbol{r} ; \boldsymbol{r}_{1}, \boldsymbol{r}_{2}\right)=\frac{2 \rho(r)}{\pi r} \sum_{\ell \ell^{\prime}}[ & -f_{\ell \ell^{\prime}}^{j}(r) \alpha_{1}^{j} P_{\ell^{\prime}}^{\prime}\left(\cos \gamma_{1}\right) P_{\ell}\left(\cos \gamma_{2}\right) \\
& \left.+g_{\ell \ell^{\prime}}^{j}(r) \alpha_{2}^{j} P_{\ell}\left(\cos \gamma_{1}\right) P_{\ell^{\prime}}^{\prime}\left(\cos \gamma_{2}\right)\right],
\end{aligned}
$$

where $P^{\prime}$ is the derivative of the Legendre polynomials and

$$
\begin{aligned}
\alpha_{i}^{\theta} & =-\cos \theta_{i} \sin \theta-\sin \theta_{i} \cos \theta \cos \left(\phi-\phi_{i}\right), \\
\alpha_{i}^{\phi} & =-\sin \theta_{i} \sin \left(\phi-\phi_{i}\right)
\end{aligned}
$$

are respectively the derivative of $\cos \gamma_{i}$ with respect to $\theta$, and the derivative with respect to $\phi$ and divided by $\sin \theta$. 
3 Contribution of flows around active regions to the north-south helioseismic measurements

Let's consider $\boldsymbol{r}_{1}$ and $\boldsymbol{r}_{2}$ two foot points in the arc-to-arc geometry described by Liang et al. (2017) and used in this paper. Let's denote $\left(\theta_{0}, \phi_{0}\right)$ the colatitude and longitude of the midpoint, $\Delta$ the separation distance between the foot points, and $\psi$ between a meridian and the ray path connecting the paired points on the arcs. Then we rewrite the flow kernels as

$$
\mathbf{K}\left(\boldsymbol{r} ; \boldsymbol{r}_{1}, \boldsymbol{r}_{2}\right)=\mathbf{K}\left(\boldsymbol{r} ; \theta_{0}, \phi_{0}, \Delta, \psi\right) .
$$

We use this notation in the main text in order to make the averaging of the travel-time perturbations over the arcs more explicit. 


\section{Discussion}

\subsection{Properties of flows around the average active region}

In a way similar to Braun (2019), we want to analyze the properties of the flows around the average active region to better understand their contribution to the meridional flow. In particular, we aim to determine if the change over time of the amplitude of $v_{\theta}^{\mathrm{AR}}$ depends on the magnetic flux of the active regions. We also check the presence of a retrograde flow on the poleward side of the active regions. We use the method described in Section 3.8.1.

\subsubsection{LCT data show a retrograde flow in the leading polarity}

Panel $a$ of Fig. 4.1 shows the average active region. Clear inflows are visible, mostly in the latitudinal direction. The flows converge preferably toward the trailing polarity, as seen by Löptien et al. (2017) using the same LCT data and by Braun (2019) using a helioseismic holography technique. This can partly be because it is known that, in sunspots with penumbra, the leading polarity is the location of outgoing moat flows that may inhibit the inflows. However, after performing the ensemble averaging, we do not see a moat flow but rather a retrograde flow from the leading polarity into the trailing polarity. Since the LCT velocities cannot be trusted inside strong magnetic regions, in a way similar to Löptien et al. (2017) we discard the pixels whose unsigned magnetic field, once smoothed with a Gaussian of $\sigma=4$ pixels, is over $50 \mathrm{G}$. The resulting average active region is presented in panel $b$. The inflows in the latitudinal direction also increase by this procedure. We did not use this procedure in Chapter 3, so $v_{\phi}^{\mathrm{AR}}$ presents a retrograde component coming from the inner part of the active regions. By running our procedure from Chapter 3 with this procedure, we estimated this additional azimuthal flow to reach about $-4 \mathrm{~m} / \mathrm{s}$ (yearly average) during high activity and at the center of the activity belt, while the additional inflow in the latitudinal direction reaches $0.5 \mathrm{~m} / \mathrm{s}$. The retrograde flow seen in Chapter 3 changes by about $1 \mathrm{~m} / \mathrm{s}$ for latitudes greater than $20^{\circ}$.

\subsubsection{Dependence of the flows on the total unsigned magnetic flux}

We discard here the pixels with a strong magnetic field using the procedure described in Section 4.1.1. As shown in Fig. 4.2, the total unsigned magnetic flux of most active regions lies between $1 \times 10^{21} \mathrm{Mx}$ and $5 \times 10^{22} \mathrm{Mx}$. We separate the active regions in three categories depending on their total flux. Both polarities have inflows with a maximum amplitude of about $\pm 10-15 \mathrm{~m} / \mathrm{s}$. The maximum arises at $\pm 3^{\circ}-5^{\circ}$ from the center. The inflows are overall stronger by a few $\mathrm{m} / \mathrm{s}$ on the poleward side. They extend up to 

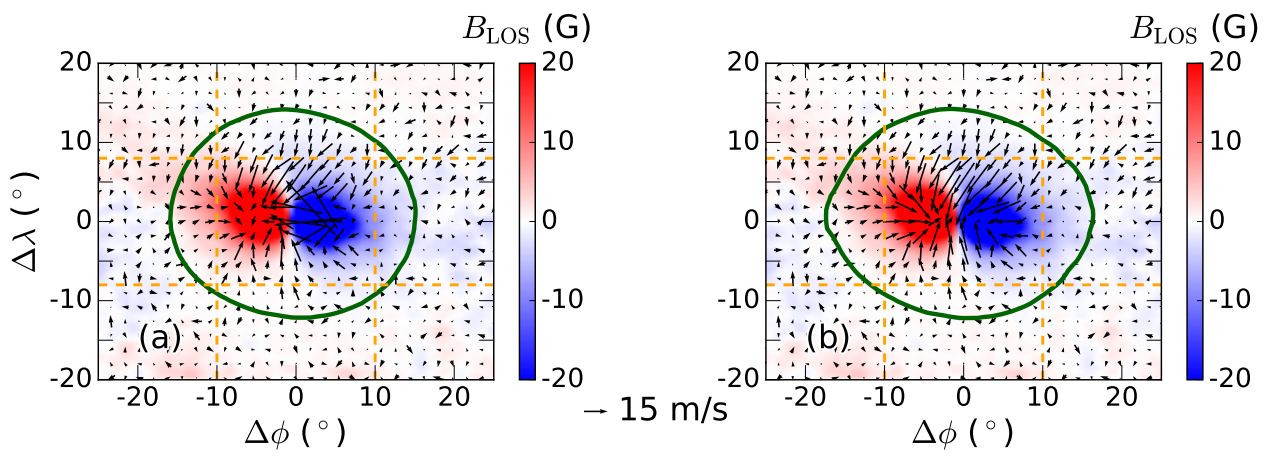

Figure 4.1: Average active region. Panel $a$ : When keeping the flows inside the active regions. Panel $b$ : When removing these flows. $\Delta \lambda$ and $\Delta \phi$ are the angular distances to the center of the active region, respectively in the latitudinal and in the longitudinal directions. $\Delta \lambda>0^{\circ}$ is poleward, $\Delta \phi>0^{\circ}$ is prograde. The orange dashed lines delimit the averaging ranges for the longitudinal and latitudinal cuts in Fig. 4.2. The green line delimits the region of $90 \%$ accuracy of the contour defined in Chapter 3, with $d=10^{\circ}$ and $b=3.5 \mathrm{G}$.

$10^{\circ}-15^{\circ}$ away from the active region center. The inflows become stronger by as much as $5 \mathrm{~m} / \mathrm{s}$ as the magnetic flux increases, with the exception of the equatorward-side inflows in the leading polarity.

In the $\phi$ direction, at $\Delta \lambda=0^{\circ}$, the flow is prograde for the trailing polarity and retrograde for the leading polarity, with an amplitude of about $\pm 5 \mathrm{~m} / \mathrm{s}$. This is consistent with an inflow signal in the $\phi$ direction. For $\Delta \lambda>0^{\circ}$, both polarities have an additional retrograde flow of amplitude $\simeq-4 \mathrm{~m} / \mathrm{s}$. As a consequence, the maximum retrograde flow in the leading polarity is shifted to $\Delta \lambda=1^{\circ}-3^{\circ}$ with an amplitude of about $-12 \mathrm{~m} / \mathrm{s}$. On average over both polarities, the net flow is retrograde up until $15^{\circ}$ on the poleward side and with a maximal amplitude of about $-8 \mathrm{~m} / \mathrm{s}$ for the highest flux range. The presence of a retrograde flow on the poleward side of the active regions confirms the findings by Braun (2019) and our result in Chapter 3.

\subsubsection{Effect of the flows around the selected active regions on the solar-cycle variation of the meridional flow}

The procedure described in this chapter allowed us to detect active regions with a total unsigned magnetic flux greater than $10^{21} \mathrm{Mx}$. Here, we aim to quantify the contribution of the flows around these active regions to the residuals measured in Chapter 3. To do so, we draw a box spanning $20^{\circ}$ in longitude and $10^{\circ}$ in latitude around the active regions. We then map back this contour to the CCD frame in order to compute the background flows, then the residuals. The contour is shown in Fig. 4.3. In this example, the present contour is only drawn around the active regions but not around decaying active regions nor around the diffuse field, unlike the contour used in Chapter 3. The results are shown in Fig. 4.4. Clearly, the flows for both contours present similar features. $v_{\theta}^{\mathrm{AR}}$ is high in 2011 in the north and until 2015 in the south for both. $v_{\phi}^{\mathrm{AR}}$ is negative during the maximum of magnetic activity for both. However, the amplitude of $v_{\theta}^{\mathrm{AR}}$ is less by more than $3 \mathrm{~m} / \mathrm{s}$ 

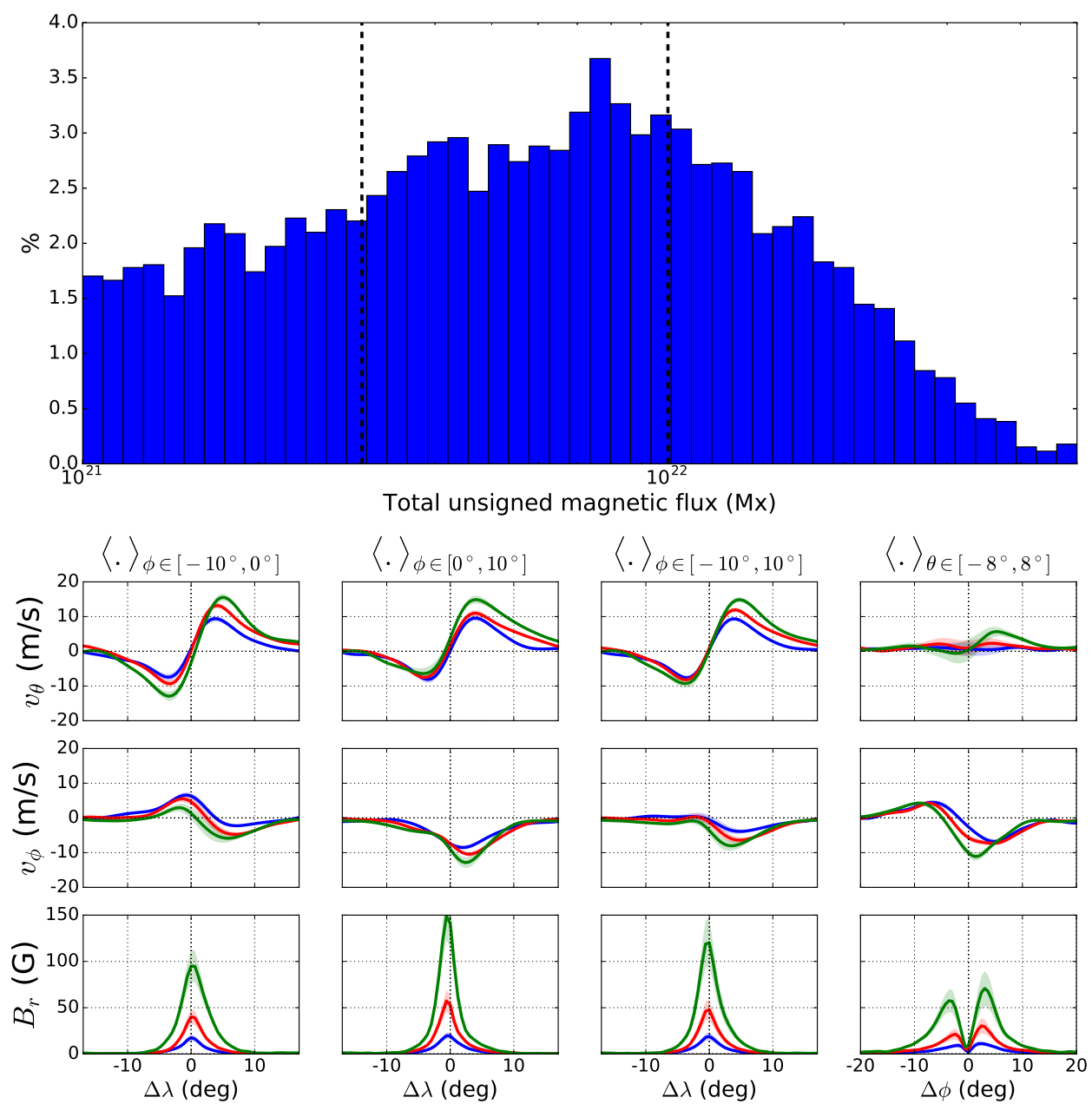

Figure 4.2: Top: Distribution of the detected active regions, based on their total unsigned magnetic flux. The 50 bins are distributed in a logarithmic way in the range $[0.1,5] \times 10^{22}$ Mx. The vertical dashed lines delimit the regions $\left[0.1 \times 10^{22}, 0.3 \times 10^{22}\right] \mathrm{Mx},\left[0.3 \times 10^{22}, 1 \times\right.$ $\left.10^{22}\right] \mathrm{Mx}$ and $\left[1 \times 10^{22}, \infty\left[\mathrm{Mx}\right.\right.$. Bottom: Flows for the flux ranges $\left[0.1 \times 10^{22}, 0.3 \times 10^{22}\right] \mathrm{Mx}$ (blue), $\left[0.3 \times 10^{22}, 1 \times 10^{22}\right] \mathrm{Mx}$ (red) and $\left[1 \times 10^{22}, \infty[\mathrm{Mx}\right.$ (green). The first column starting from the left is a longitudinal average over the trailing polarity, the second column over the leading polarity and the third column over both polarities. The fourth column is a latitudinal average. The averages are computed between the orange dashed lines in Fig. 4.1. $v_{\theta}$ is positive in the $-\Delta \lambda$ direction, $v_{\phi}$ in the $+\Delta \phi$ direction. The flows have been smoothed with a 2D Gaussian with $\sigma=2^{\circ}$ for better visualization. The last row shows the averaged magnetic field.

around 2011 in the north and around 2014 in the south. The maximum amplitude also occurs earlier in the South. This tends to suggest that the diffuse field, which the present contour ignored, is also the location of inflows, and that these play an important role in the temporal variation of the meridional flow. 
$B_{\mathrm{LOS}}(\mathrm{G})$

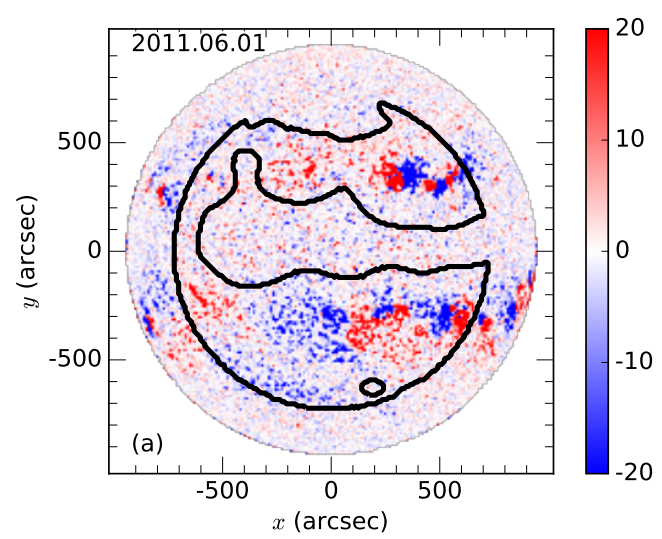

$B_{\mathrm{LOS}}(\mathrm{G})$

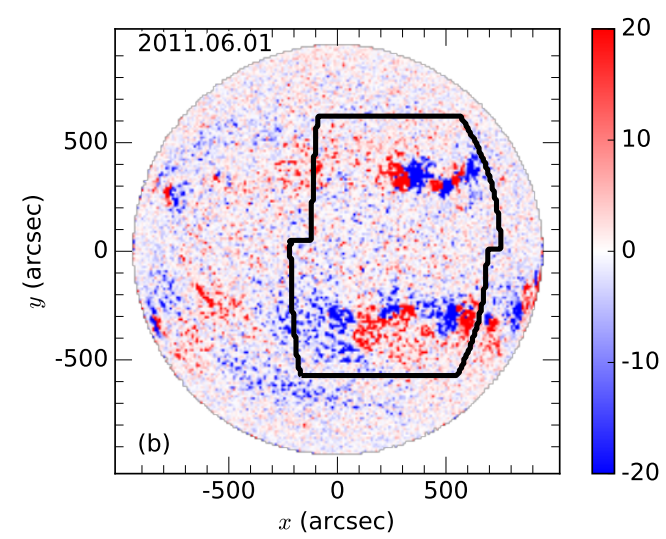

Figure 4.3: Example of the contour used to define the background, superimposed on the HMI magnetogram at noon on June 1, 2011. Panel a: contour presented in Chapter 3. Panel b: present contour.
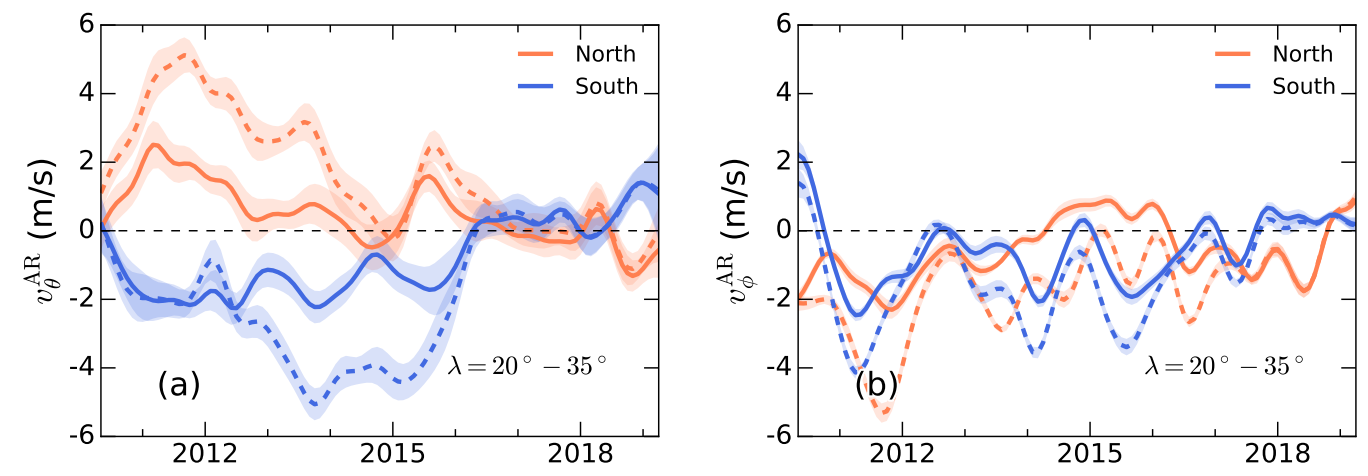

Figure 4.4: Longitudinally-averaged active-region flows $v_{\theta}^{\mathrm{AR}}$ (panel $a$ ) and $v_{\phi}^{\mathrm{AR}}$ (panel $b$ ). The dashed lines are the flows presented in Chapter 3. The solid lines are the flows computed using the detection procedure described in this chapter. The shaded areas represent the standard error of the mean over the latitudinal range.

\subsubsection{Conclusion of the analysis}

We studied the properties of the flows surrounding well-separated active regions with total unsigned magnetic flux greater than $10^{21} \mathrm{Mx}$. The signal that we see, after performing the ensemble averaging, inside the leading polarity of large active regions, has little effect on the latitudinal component of the active-region flows in Chapter 3 and, therefore, on the associated forward travel-time perturbations. We confirmed previous findings of a retrograde flow flanking the poleward side of active regions, even for regions with a small magnetic flux. However, the inflows around the regions that we analyzed account only for a small part of the flow residuals measured in Chapter 3, so there may be a large contribution from smaller regions and from the diffuse magnetic field. 


\subsection{Conclusion and outlook}

The main goal of this thesis was to study the solar dynamics near the surface. It focused on two types of varying flows, on different time scales, which affect helioseismic observations. The main conclusions are:

\subsubsection{Propagation of acoustic waves through turbulent convection}

- For a 1D set-up with random sound speed perturbations, we found that the Keller theory works best to represent the wave propagation through a medium resembling solar granulation. In particular, it can be easily derived when the medium varies with time. We showed, perhaps surprisingly, that a frozen-medium approximation, although seemingly unjustified, actually works well to retrieve the effective wave speed. More precisely, one can use a snapshot of granulation to model the wave propagation and find a wave speed with a 5\% accuracy. The attenuation however is off by about $30 \%$. We detect coda waves in the variance of the wave field. These waves are commonly studied in terrestrial seismology but have yet to be detected in helioseismology. In our set-up, a small part of the coda propagates forward and results from multiple scattering, while most of it propagates backward and results from a reflection somewhere in the medium (single back-scattering).

- The next step is to extend this study to the propagation of seismic waves through realizations of a solar-like random medium with the help of the Montjoie solver (Gizon et al. 2017). Possible improvements include moving to three dimensions in a stratified medium, and adding flows. So far, sound speed fluctuations can be seen only as isotropic flow perturbations, but solar granulation and convection in general are anisotropic. The passage to multiple dimensions will bring, in particular, an additional attenuation because of geometrical spreading. Finally, one needs to connect the wave field to the cross-covariance function used in time-distance helioseismology. In the time domain,

$$
C\left(\mathbf{r}_{1}, \mathbf{r}_{2}, t\right)=\frac{1}{T} \int_{-T / 2}^{T / 2} \Phi\left(\mathbf{r}_{1}, t^{\prime}\right) \Phi\left(\mathbf{r}_{2}, t^{\prime}+t\right) \mathrm{dt}^{\prime},
$$

where $\Phi\left(\mathbf{r}_{1}, t^{\prime}\right)$ denotes the Doppler signal observed at point $\mathbf{r}_{\mathbf{1}}$ and time $t^{\prime}, \Phi\left(\mathbf{r}_{2}, t^{\prime}+\right.$ $t$ ) the one observed at point $\mathbf{r}_{2}$ and time $t^{\prime}+t$, and $T$ is the duration of the observation. $t$ represents here a time lag. The cross-covariance is used as a proxy to observe the wave packets propagating between $\mathbf{r}_{1}$ and $\mathbf{r}_{2}$, so it should also satisfy a wave equation.

\subsubsection{Near-surface inflows around active regions}

- We measured the contribution of near-surface activity-related flows to the timeaveraged meridional circulation, by identifying and isolating these flows in surface flow measurements. We found that, if we assume that the active-region flows are constant with depth, the amplitude of the north-south helioseismic travel-time perturbations associated with these flows reaches $\pm 0.3 \mathrm{~s}$ during solar maximum and 
for separation distances $6^{\circ}-18^{\circ}$. When masking magnetic pixels that create systematic effects in time-distance measurements, this amplitude drops significantly. Therefore, the active-region inflows do not explain in full the solar-cycle variations seen in the helioseismic measurements of the meridional circulation.

- One direct application of isolating the active-region inflows may be to improve on the inversions of the radial profile of meridional circulation down to the bottom of the convection zone, which has been under debate for years. A cleaner surface flow measurement can be given as an input to the inversions, with the contributions of the global and local cells being clearly separated. 


\section{Bibliography}

Aki, K.: 1969, J. Geophys. Res. 74(2), 615

Aki, K. and Chouet, B.: 1975, J. Geophys. Res. 80(23), 3322

Aki, K. and Richards, P. G.: 2002, Quantitative Seismology, 2nd Ed., University Science Books

Aki, K. and Wu, R.-S.: 1988, Scattering and Attenuation of Seismic Waves, Part I, Springer

Antia, H. M. and Basu, S.: 2000, ApJ 541(1), 442

Antia, H. M. and Basu, S.: 2001, ApJ 559(1), L67

Ball, W. H., Beeck, B., Cameron, R. H., and Gizon, L.: 2016, AE $A$ 592, A159

Ball, W. H. and Gizon, L.: 2017, AEFA 600, A128

Balmforth, N. J.: 1992a, MNRAS 255, 603

Balmforth, N. J.: 1992b, MNRAS 255, 632

Balmforth, N. J.: 1992c, MNRAS 255, 639

Baran, O. A.: 2013, Advances in Astronomy and Space Physics 3, 89

Barekat, A., Schou, J., and Gizon, L.: 2014, AE्FA 570, L12

Basu, S.: 2010, ApESSS 328(1-2), 43

Basu, S. and Antia, H. M.: 2000, Sol. Phys. 192, 469

Bhattacharya, J., Hanasoge, S., and Antia, H. M.: 2015, ApJ 806, 246

Bogdan, T. J.: 1989, ApJ 339, 1132

Böning, V. G. A., Roth, M., Jackiewicz, J., and Kholikov, S.: 2017, ApJ 845(1), 2

Bourret, R. C.: 1963, in Volume 12 of Applied Scientific Research, Section A, Vol. 12 of Applied Scientific Research, p. 223

Braun, D. C.: 2019, ApJ 873(1), 94 
Brown, T. M.: 1984, Science 226(4675), 687

Brown, T. M., Christensen-Dalsgaard, J., Dziembowski, W. A., Goode, P., Gough, D. O., and Morrow, C. A.: 1989, ApJ 343, 526

Burston, R., Gizon, L., and Birch, A. C.: 2015, Space Sci. Rev. 196(1-4), 201

Cameron, R. H. and Schüssler, M.: 2010, ApJ 720(2), 1030

Cameron, R. H. and Schüssler, M.: 2012, AEFA 548, A57

Chen, R. and Zhao, J.: 2017, ApJ 849(2), 144

Chou, D.-Y. and Dai, D.-C.: 2001, ApJ 559(2), L175

Chou, D.-Y. and Ladenkov, O.: 2005, ApJ 630(2), 1206

Christensen-Dalsgaard, J.: 2002, Reviews of Modern Physics 74(4), 1073

Christensen-Dalsgaard, J., Dappen, W., Ajukov, S. V., Anderson, E. R., Antia, H. M., Basu, S., Baturin, V. A., Berthomieu, G., Chaboyer, B., Chitre, S. M., Cox, A. N., Demarque, P., Donatowicz, J., Dziembowski, W. A., Gabriel, M., Gough, D. O., Guenther, D. B., Guzik, J. A., Harvey, J. W., Hill, F., Houdek, G., Iglesias, C. A., Kosovichev, A. G., Leibacher, J. W., Morel, P., Proffitt, C. R., Provost, J., Reiter, J., Rhodes, E. J., J., Rogers, F. J., Roxburgh, I. W., Thompson, M. J., and Ulrich, R. K.: 1996, Science 272(5266), 1286

Christensen-Dalsgaard, J., Duvall, T. L., J., Gough, D. O., Harvey, J. W., and Rhodes, E. J., J.: 1985, Nature 315(6018), 378

Christensen-Dalsgaard, J., Gough, D. O., and Thompson, M. J.: 1991, ApJ 378, 413

Christensen-Dalsgaard, J. and Schou, J.: 1988, in E. J. Rolfe (ed.), Seismology of the Sun and Sun-Like Stars, Vol. 286 of ESA Special Publication, pp 149-153

De Rosa, M. L. and Schrijver, C. J.: 2006, in K. Fletcher and M. Thompson (eds.), Proceedings of SOHO 18/GONG 2006/HELAS I, Beyond the spherical Sun, Vol. 624 of ESA Special Publication, p. 12

Delache, P. and Fossat, E.: 1988, in E. J. Rolfe (ed.), Seismology of the Sun and Sun-Like Stars, Vol. 286 of ESA Special Publication, pp 671-672

Deubner, F. L.: 1975, AEFA 44(2), 371

Dikpati, M. and Gilman, P. A.: 2006, ApJ 649(1), 498

Dikpati, M. and Gilman, P. A.: 2009, Flux-Transport Solar Dynamos, Vol. 32, p. 67

Duvall, T. L., J.: 1979, Sol. Phys. 63(1), 3

Duvall, T. L., J.: 1982, Nature 300(5889), 242 
Duvall, T. L., J., Dziembowski, W. A., Goode, P. R., Gough, D. O., Harvey, J. W., and Leibacher, J. W.: 1984, Nature 310(5972), 22

Duvall, T. L., J. and Harvey, J. W.: 1984, Nature 310, 19

Duvall, T. L., J., Harvey, J. W., and Pomerantz, M. A.: 1986, Nature 321(6069), 500

Duvall, T. L., J., Jefferies, S. M., Harvey, J. W., and Pomerantz, M. A.: 1993, Nature 362(6419), 430

Duvall, T. L., J., Kosovichev, A. G., and Murawski, K.: 1998, ApJ 505(1), L55

Fisher, G. H. and Welsch, B. T.: 2008, in R. Howe, R. W. Komm, K. S. Balasubramaniam, and G. J. D. Petrie (eds.), Subsurface and Atmospheric Influences on Solar Activity, Vol. 383 of Astronomical Society of the Pacific Conference Series, p. 373

Fossat, E., Boumier, P., Corbard, T., Provost, J., Salabert, D., Schmider, F. X., Gabriel, A. H., Grec, G., Renaud, C., Robillot, J. M., Roca-Cortés, T., Turck-Chièze, S., Ulrich, R. K., and Lazrek, M.: 2017, AEFA 604, A40

Fournier, D., Hanson, C. S., Gizon, L., and Barucq, H.: 2018, AEA 616, A156

Getling, A. V., Kosovichev, A. G., and Zhao, J.: 2021, ApJ 908(2), L50

Giles, P. M., Duvall, T. L., Scherrer, P. H., and Bogart, R. S.: 1997, Nature 390(6655), 52

Gizon, L.: 2003, Ph.D. thesis, STANFORD UNIVERSITY

Gizon, L.: 2004, Sol. Phys. 224(1-2), 217

Gizon, L., Barucq, H., Duruflé, M., Hanson, C. S., Leguèbe, M., Birch, A. C., Chabassier, J., Fournier, D., Hohage, T., and Papini, E.: 2017, AEFA 600, A35

Gizon, L. and Birch, A. C.: 2004, ApJ 614(1), 472

Gizon, L. and Birch, A. C.: 2005, Living Reviews in Solar Physics 2(1), 6

Gizon, L., Birch, A. C., and Spruit, H. C.: 2010, ARAEAA 48, 289

Gizon, L., Cameron, R. H., Pourabdian, M., Liang, Z.-C., Fournier, D., Birch, A. C., and Hanson, C. S.: 2020, Science 368(6498), 1469

Gizon, L., Duvall, T. L., J., and Larsen, R. M.: 2001, in P. Brekke, B. Fleck, and J. B. Gurman (eds.), Recent Insights into the Physics of the Sun and Heliosphere: Highlights from SOHO and Other Space Missions, Vol. 203 of IAU Symposium, p. 189

Gizon, L. and Rempel, M.: 2008, Sol. Phys. 251(1-2), 241

Goldreich, P. and Keeley, D. A.: 1977, ApJ 212, 243

Goldreich, P. and Murray, N.: 1994, ApJ 424, 480

González Hernández, I., Howe, R., Komm, R., and Hill, F.: 2010, ApJ 713(1), L16 
González Hernández, I., Kholikov, S., Hill, F., Howe, R., and Komm, R.: 2008, Sol. Phys. 252(2), 235

Gottschling, N., Schunker, H., Birch, A. C., Löptien, B., and Gizon, L.: 2021, arXiv e-prints p. arXiv:2105.10501

Gough, D. and Toomre, J.: 1991, ARAEA 29, 627

Gough, D. O.: 1977, ApJ 214, 196

Haber, D. A., Hindman, B. W., Toomre, J., Bogart, R. S., Thompson, M. J., and Hill, F.: 2000, Sol. Phys. 192, 335

Haber, D. A., Hindman, B. W., Toomre, J., and Thompson, M. J.: 2004, Sol. Phys. 220(2), 371

Hanasoge, S. M., Gizon, L., and Bal, G.: 2013, ApJ 773(2), 101

Hart, A. B.: 1954, MNRAS 114, 17

Harvey, J. W., Hill, F., Hubbard, R. P., Kennedy, J. R., Leibacher, J. W., Pintar, J. A., Gilman, P. A., Noyes, R. W., Title, A. M., Toomre, J., Ulrich, R. K., Bhatnagar, A., Kennewell, J. A., Marquette, W., Patron, J., Saa, O., and Yasukawa, E.: 1996, Science 272(5266), 1284

Hathaway, D. H.: 2012, ApJ 760(1), 84

Hathaway, D. H. and Rightmire, L.: 2010, Science 327(5971), 1350

Hathaway, D. H. and Rightmire, L.: 2011, ApJ 729(2), 80

Hindman, B., Gizon, L., Haber, D., Duval, Thomas, J., and Toomre, J.: 2003, in H. Sawaya-Lacoste (ed.), GONG+ 2002. Local and Global Helioseismology: the Present and Future, Vol. 517 of ESA Special Publication, pp 299-302

Hindman, B. W., Haber, D. A., and Toomre, J.: 2009, ApJ 698(2), 1749

Hirzberger, J., Gizon, L., Solanki, S. K., and Duvall, T. L.: 2008, Sol. Phys. 251(1-2), 417

Houdek, G.: 2006, arXiv e-prints pp astro-ph/0612024

Houdek, G., Balmforth, N. J., Christensen-Dalsgaard, J., and Gough, D. O.: 1999, AEAA 351, 582

Houdek, G. and Dupret, M.-A.: 2015, Living Reviews in Solar Physics 12(1), 8

Houdek, G., Trampedach, R., Aarslev, M. J., and Christensen-Dalsgaard, J.: 2017, $M N$ RAS 464(1), L124

Howard, R. and Labonte, B. J.: 1980, ApJ 239, L33

Howard, R. F., Harvey, J. W., and Forgach, S.: 1990, Sol. Phys. 130(1-2), 295 
Howe, M. S.: 1971, Journal of Fluid Mechanics 45, 785

Howe, R., Christensen-Dalsgaard, J., Hill, F., Komm, R. W., Larsen, R. M., Schou, J., Thompson, M. J., and Toomre, J.: 2000, ApJ 533(2), L163

Keller, J. B.: 1964, in R. A. M. S. Providence (ed.), Volume 16 of Proceedings of symposia in applied mathematics, Vol. 16 of Proceedings of symposia in applied mathematics, $\mathrm{p}$. 145

Kitchatinov, L. L.: 2011, in Astronomical Society of India Conference Series, Vol. 2 of Astronomical Society of India Conference Series, pp 71-80

Kitchatinov, L. L.: 2016, Geomagnetism and Aeronomy 56(8), 945

Komm, R., González Hernández, I., Howe, R., and Hill, F.: 2015, Sol. Phys. 290(11), 3113

Komm, R., Howe, R., González Hernández, I., and Hill, F.: 2014, Sol. Phys. 289(9), 3435

Komm, R., Howe, R., González Hernández, I., Hill, F., Haber, D., Hindman, B., and Corbard, T.: 2004, in D. Danesy (ed.), SOHO 14 Helio- and Asteroseismology: Towards a Golden Future, Vol. 559 of ESA Special Publication, p. 520

Komm, R., Howe, R., and Hill, F.: 2018, Sol. Phys. 293(10), 145

Komm, R., Howe, R., and Hill, F.: 2020, Sol. Phys. 295(3), 47

Komm, R. W.: 1994, Sol. Phys. 149(2), 417

Komm, R. W., Howard, R. F., and Harvey, J. W.: 1993, Sol. Phys. 147(2), 207

Kosovichev, A. G. and Schou, J.: 1997, ApJ 482(2), L207

Larson, T. P. and Schou, J.: 2018, Sol. Phys. 293(2), 29

Legendre, G.: 2003, Ph.D. thesis, universite paris vi

Leibacher, J. W. and Stein, R. F.: 1971, Astrophys. Lett. 7, 191

Leibacher, J. W. and Stein, R. F.: 1981, Oscillations and pulsations., Vol. 450, pp 263287

Leighton, R. B., Noyes, R. W., and Simon, G. W.: 1962, ApJ 135, 474

Liang, Z.-C., Birch, A. C., Duvall, Thomas L., J., Gizon, L., and Schou, J.: 2017, AEFA 601, A46

Liang, Z.-C. and Chou, D.-Y.: 2015, ApJ 805(2), 165

Liang, Z.-C., Gizon, L., Birch, A. C., Duvall, T. L., and Rajaguru, S. P.: 2018, AE्FA 619, A99

Libbrecht, K. G.: 1988, ApJ 334, 510 
Libbrecht, K. G.: 1989, ApJ 336, 1092

Libbrecht, K. G. and Woodard, M. F.: 1991, Science 253(5016), 152

Libbrecht, K. G., Woodard, M. F., and Kaufman, J. M.: 1990, ApJS 74, 1129

Lin, C.-H. and Chou, D.-Y.: 2018, ApJ 860(1), 48

Lisle, J. and Toomre, J.: 2004, in D. Danesy (ed.), SOHO 14 Helio- and Asteroseismology: Towards a Golden Future, Vol. 559 of ESA Special Publication, p. 556

Liu, Y., Hoeksema, J. T., Scherrer, P. H., Schou, J., Couvidat, S., Bush, R. I., Duvall, T. L., Hayashi, K., Sun, X., and Zhao, X.: 2012, Sol. Phys. 279(1), 295

Löptien, B., Birch, A. C., Duvall, T. L., Gizon, L., Proxauf, B., and Schou, J.: 2017, AEFA 606, A28

Löptien, B., Birch, A. C., Duvall, T. L., Gizon, L., and Schou, J.: 2016, AEFA 590, A130

Lynden-Bell, D. and Ostriker, J. P.: 1967, MNRAS 136, 293

Mandal, K., Hanasoge, S. M., Rajaguru, S. P., and Antia, H. M.: 2018, ApJ 863(1), 39

Mędrek, M., Murawski, K., and Roberts, B.: 1999, AEFA 349, 312

Meunier, N.: 1999, ApJ 527(2), 967

Müller, G., Roth, M., and Korn, M.: 1992, Geophysical Journal International 110(1), 29

Murawski, K., Duvall, T. L., J., and Kosovichev, A. G.: 1998, in S. Korzennik (ed.), Structure and Dynamics of the Interior of the Sun and Sun-like Stars, Vol. 418 of ESA Special Publication, p. 825

Murawski, K. and Roberts, B.: 1993a, AE्EA 272, 595

Murawski, K. and Roberts, B.: 1993b, AE्EA 272, 601

Nordlund, Å., Stein, R. F., and Asplund, M.: 2009, Living Reviews in Solar Physics 6(1), 2

November, L. J. and Simon, G. W.: 1988, ApJ 333, 427

Papanicolaou, G. C. and Varadhan, S. R. S.: 1982, Diffusion with random coefficients, Kallianpur, G. and Krishnaiah, P.R. and Ghosh, J.K. (North Holland)

Rempel, M.: 2006, ApJ 647(1), 662

Rempel, M.: 2007, ApJ 655(1), 651

Rieutord, M., Meunier, N., Roudier, T., Rondi, S., Beigbeder, F., and Parès, L.: 2008, $A \mathcal{E} A$ 479(1), L17

Rieutord, M. and Rincon, F.: 2010, Living Reviews in Solar Physics 7(1), 2 
Rieutord, M., Roudier, T., Rincon, F., Malherbe, J. M., Meunier, N., Berger, T., and Frank, Z.: 2010, AEA 512, A4

Rincon, F. and Rieutord, M.: 2018, Living Reviews in Solar Physics 15(1), 6

Rosenthal, C. S., Christensen-Dalsgaard, J., Nordlund, Å., Stein, R. F., and Trampedach, R.: 1999, AEFA 351, 689

Roth, M. and Stix, M.: 2001, in A. Wilson and P. L. Pallé (eds.), SOHO 10/GONG 2000 Workshop: Helio- and Asteroseismology at the Dawn of the Millennium, Vol. 464 of ESA Special Publication, pp 243-246

Roth, M. and Stix, M.: 2003, AEFA 405, 779

Rytov, S. M., Kravtsov, Y. A., and Tatarskii, V. I.: 1989a, Principles of statistical radiophysics. 4. Wave propagation through random media., Springer

Rytov, S. M., Kravtsov, Y. A., and Tatarskii, V. I.: 1989b, Priniciples of statistical radiophysics. 3. Elements of random fields., Springer

Sato, H., Fehler, M. C., and Maeda, T.: 2012, Seismic Wave Propagation and Scattering in the Heterogeneous Earth, 2nd Ed., Springer

Scherrer, P. H., Bogart, R. S., Bush, R. I., Hoeksema, J. T., Kosovichev, A. G., Schou, J., Rosenberg, W., Springer, L., Tarbell, T. D., Title, A., Wolfson, C. J., Zayer, I., and MDI Engineering Team: 1995, Sol. Phys. 162(1-2), 129

Scherrer, P. H., Schou, J., Bush, R. I., Kosovichev, A. G., Bogart, R. S., Hoeksema, J. T., Liu, Y., Duvall, T. L., Zhao, J., Title, A. M., Schrijver, C. J., Tarbell, T. D., and Tomczyk, S.: 2012, Sol. Phys. 275(1-2), 207

Schou, J.: 1999, ApJ 523(2), L181

Schou, J.: 2003, ApJ 596(2), L259

Schou, J., Antia, H. M., Basu, S., Bogart, R. S., Bush, R. I., Chitre, S. M., ChristensenDalsgaard, J., Di Mauro, M. P., Dziembowski, W. A., Eff-Darwich, A., Gough, D. O., Haber, D. A., Hoeksema, J. T., Howe, R., Korzennik, S. G., Kosovichev, A. G., Larsen, R. M., Pijpers, F. P., Scherrer, P. H., Sekii, T., Tarbell, T. D., Title, A. M., Thompson, M. J., and Toomre, J.: 1998, ApJ 505, 390

Schou, J. and Birch, A. C.: 2020, arXiv e-prints p. arXiv:2004.13548

Schuessler, M.: 1981, AEFA 94(2), L17

Schunker, H., Schou, J., Gaulme, P., and Gizon, L.: 2018, Sol. Phys. 293(6), 95

Sheeley, N. R., J.: 1992, The Flux-Transport Model and Its Implications, Vol. 27 of Astronomical Society of the Pacific Conference Series, p. 1

Snodgrass, H. B. and Ulrich, R. K.: 1990, ApJ 351, 309 
Sonoi, T., Samadi, R., Belkacem, K., Ludwig, H. G., Caffau, E., and Mosser, B.: 2015, $A \mathcal{E} A$ 583, A 112

Spruit, H. C.: 2003, Sol. Phys. 213(1), 1

Tatarskii, V. I.: 1971, The effects of the turbulent atmosphere on wave propagation

Thompson, M. J., Christensen-Dalsgaard, J., Miesch, M. S., and Toomre, J.: 2003, ARAE $A$ 41, 599

Thompson, M. J., Toomre, J., Anderson, E. R., Antia, H. M., Berthomieu, G., Burtonclay, D., Chitre, S. M., Christensen-Dalsgaard, J., Corbard, T., De Rosa, M., Genovese, C. R., Gough, D. O., Haber, D. A., Harvey, J. W., Hill, F., Howe, R., Korzennik, S. G., Kosovichev, A. G., Leibacher, J. W., Pijpers, F. P., Provost, J., Rhodes, E. J., J., Schou, J., Sekii, T., Stark, P. B., and Wilson, P. R.: 1996, Science 272(5266), 1300

Title, A. M., Tarbell, T. D., Topka, K. P., Ferguson, S. H., Shine, R. A., and SOUP Team: 1989, ApJ 336, 475

Toomre, J., Christensen-Dalsgaard, J., Howe, R., Larsen, R. M., Schou, J., and Thompson, M. J.: 2000, Sol. Phys. 192, 437

Ulrich, R. K.: 1970, ApJ 162, 993

Unno, W.: 1967, PASJ 19, 140

van der Baan, M.: 2001, Geophysical Journal International 145(3), 631

Vorontsov, S. V., Christensen-Dalsgaard, J., Schou, J., Strakhov, V. N., and Thompson, M. J.: 2002, Science 296(5565), 101

Wang, Y. M., Sheeley, N. R., J., and Nash, A. G.: 1991, ApJ 383, 431

Welsch, B. T., Fisher, G. H., Abbett, W. P., and Regnier, S.: 2004, ApJ 610(2), 1148

Woodard, M. F.: 1997, ApJ 485(2), 890

Yoshimura, H.: 1981, ApJ 247, 1102

Zhao, J., Bogart, R. S., Kosovichev, A. G., Duvall, T. L., J., and Hartlep, T.: 2013, ApJ 774(2), L29

Zhao, J. and Kosovichev, A. G.: 2004, ApJ 603(2), 776

Zhao, J., Kosovichev, A. G., and Bogart, R. S.: 2014, ApJ 789(1), L7 
Appendix 



\section{A Derivation of the second-order Born approximation for a slab of frozen random medium}

This appendix derives the second-order Born approximation for Medium 1, following the notations of the paper presented in Chapter 2. We drop however the overhead tilde that denoted the Fourier transform, for the sake of simplicity. The approximation requires the perturbation to have a compact support as it behaves as a source term for the perturbed wave field. Let's assume a localized perturbation between $X$ and $X+L$, namely,

$$
\begin{aligned}
\left\langle\delta c(x) \delta c\left(x^{\prime}\right)\right\rangle & =\epsilon^{2} c_{O}^{2} M\left(x, x^{\prime}\right)=\epsilon^{2} c_{O}^{2} f(\zeta) w(\bar{x}), \\
f(\zeta) & =e^{-|\zeta| / a} \\
w(\bar{x}) & =\Theta(\bar{x}-X)-\Theta(\bar{x}-(X+L)), \\
\zeta & =x^{\prime}-x, \\
\bar{x} & =\frac{x+x^{\prime}}{2},
\end{aligned}
$$

where $\Theta$ is the Heaviside step function. Then

$$
\begin{aligned}
& \partial_{\bar{x}} w(\bar{x})=\delta(\bar{x}-X)-\delta(\bar{x}-(X+L)), \\
& \partial_{\bar{x}}^{2} w(\bar{x})=-\frac{\delta(\bar{x}-X)}{\bar{x}}+\frac{\delta(\bar{x}-(X+L))}{\bar{x}} .
\end{aligned}
$$

Now let's use the Born approximation, valid for small perturbations $(\epsilon \ll 1)$. Using the same notations for the operators, denoting $\phi_{0}$ the unperturbed field and $\phi_{1}$ the correction such that $\phi=\phi_{0}+\phi_{1}$, the Born approximation reads

$$
\phi=\phi_{0}-L_{0}^{-1} L_{1} \phi_{0}+O\left(\epsilon^{2}\right) .
$$

Taking the average, one gets $\langle\phi\rangle=\phi_{0}+O\left(\epsilon^{2}\right)$. This means that we have to go down to the second order:

$$
\phi=\phi_{0}-L_{0}^{-1} L_{1} \phi_{0}+L_{0}^{-1} L_{1} L_{0}^{-1} L_{1} \phi_{0}-L_{0}^{-1} L_{2} \phi_{0}+O\left(\epsilon^{3}\right),
$$

which gives, once averaged,

$$
\langle\phi\rangle=\phi_{0}+L_{0}^{-1}\left\langle L_{1} L_{0}^{-1} L_{1}\right\rangle \phi_{0}-L_{0}^{-1}\left\langle L_{2}\right\rangle \phi_{0}+O\left(\epsilon^{3}\right) .
$$

We get

$$
\left\langle L_{1} L_{0}^{-1} L_{1}\right\rangle \phi_{0}(x)=4 c_{0}^{4} \epsilon^{2}\left(\left(\partial_{x}+i k_{0}\right)^{2} I_{B}(x)\right) \phi_{0}(x),
$$


A Derivation of the second-order Born approximation for a slab of frozen random medium

where

$$
\begin{aligned}
I_{B}(x) & =\int_{-\infty}^{\infty} G_{0}\left(x, x^{\prime}\right)\left[\left(\partial_{x^{\prime}}+i k_{0}\right)^{2} M\left(x, x^{\prime}\right)\right] e^{i k_{0} \zeta} d x^{\prime} \\
& =\int_{-\infty}^{\infty} G_{0}\left(x, x^{\prime}\right)\left[\left(\partial_{x^{\prime}}+i k_{0}\right)^{2} f(\zeta)\right] w(\bar{x}) e^{i k_{0} \zeta} d x^{\prime} \\
& +\int_{-\infty}^{\infty} G_{0}\left(x, x^{\prime}\right) e^{i k_{0} \zeta}\left[2 \partial_{x^{\prime}} f(\zeta) \partial_{x^{\prime}} w(\bar{x})+f(\zeta) \partial_{x^{\prime}}^{2} w(\bar{x})+2 i k_{0} f(\zeta) \partial_{x^{\prime}} w(\bar{x})\right] d x^{\prime} \\
& =\underbrace{\int_{2 X-x}^{2(X+L)-x} G_{0}\left(x, x^{\prime}\right)\left[\left(\partial_{x^{\prime}}+i k_{0}\right)^{2} f(\zeta)\right] e^{i k_{0} \zeta} d x^{\prime}}_{I_{B, 1}}+\underbrace{\text { boundary terms }}_{I_{B, 2}},
\end{aligned}
$$

where the boundary terms are introduced by the derivatives of $w$. Then, calculations lead to:

$$
\begin{aligned}
I_{B, 1}(x) & =\frac{i}{2 c_{0}^{2} k_{0} a}\left(1+\frac{\left(k_{0} a\right)^{2}}{2 i k_{0} a-1}\right)\left(e^{2 \frac{2 i k_{0} a-1}{a} L}-1\right) e^{2 \frac{2 i k_{0} a-1}{a}(X-x)} \text { if } x<X, \\
& =\frac{i}{2 c_{0}^{2} k_{0} a}\left[\left(1+\frac{\left(k_{0} a\right)^{2}}{2 i k_{0} a-1}\right)\left(e^{2 \frac{2 i_{0} a-1}{a}(X+L-x)}-1\right)-\left(1+2 i k_{0} a-\left(k_{0} a\right)^{2}\right)\left(1-e^{2 \frac{X-x}{a}}\right)+2\right] \\
& \text { if } X \leq x \leq X+L, \\
& =-\frac{i}{2 c_{0}^{2} k_{0} a}\left(1+2 i k_{0} a-\left(k_{0} a\right)^{2}\right)\left(e^{2 \frac{L}{a}}-1\right) e^{-2 \frac{x-X}{a}} \text { if } x>X+L .
\end{aligned}
$$

The boundary terms read:

$$
\begin{aligned}
I_{B, 2}(x) & =\frac{1}{4 c_{0}^{2}} e^{2 \frac{2 i k_{0} a-1}{a}(X-x)}\left(e^{2 \frac{2 i k_{0} a-1}{a} L}\left[-\frac{i}{2 k_{0}(X+L)}-\frac{2 i}{k_{0} a}-2\right]+\left[\frac{i}{2 k_{0} X}+\frac{2 i}{k_{0} a}+2\right]\right) \\
& \text { if } x<X \\
& =\frac{1}{4 c_{0}^{2}} e^{2 \frac{2 i k_{0} a-1}{a}(X+L-x)}\left[-\frac{i}{2 k_{0}(X+L)}-\frac{2 i}{k_{0} a}-2\right]+e^{-\frac{2}{a}(x-X)}\left[\frac{i}{2 k_{0} X}-\frac{2 i}{k_{0} a}+2\right] \\
& \text { if } X \leq x \leq X+L, \\
& =\frac{1}{4 c_{0}^{2}} e^{-\frac{2}{a}(x-X)}\left(e^{\frac{2}{a} L}\left[-\frac{i}{2 k_{0}(X+L)}+\frac{2 i}{k_{0} a}-2\right]+\left[\frac{i}{2 k_{0} X}-\frac{2 i}{k_{0} a}+2\right]\right) \\
& \text { if } X+L<x .
\end{aligned}
$$

It can be condensed in

$$
\begin{aligned}
I_{B, i}(x) & =K_{0}^{(i)} e^{\alpha x} \text { if } x<X, \\
& =K_{1}^{(i)} e^{\alpha x}+K_{2}^{(i)} e^{\beta x}+K_{3}^{(i)} \text { if } X \leq x \leq X+L, \\
& =K_{4}^{(i)} e^{\beta x} \text { if } x>X+L,
\end{aligned}
$$


where $i \in\{1,2\}$ and

$$
\begin{aligned}
K_{0}^{(1)} & =\frac{i}{2 c_{0}^{2} k_{0} a}\left(1+\frac{\left(k_{0} a\right)^{2}}{2 i k_{0} a-1}\right)\left(e^{-\alpha L}-1\right) e^{-\alpha X}, \\
K_{1}^{(1)} & =\frac{i}{2 c_{0}^{2} k_{0} a}\left(1+\frac{\left(k_{0} a\right)^{2}}{2 i k_{0} a-1}\right) e^{-\alpha(X+L)}, \\
K_{2}^{(1)} & =\frac{i}{2 c_{0}^{2} k_{0} a}\left(1+i k_{0} a\right)^{2} e^{-\beta X}, \\
K_{3}^{(1)} & =\frac{i}{2 c_{0}^{2} k_{0} a}\left[-\frac{\left(k_{0} a\right)^{2}}{2 i k_{0} a-1}+\left(k_{0} a\right)^{2}-2 i k_{0} a\right], \\
K_{4}^{(1)} & =-\frac{i}{2 c_{0}^{2} k_{0} a}\left(1+i k_{0} a\right)^{2}\left(e^{-\beta L}-1\right) e^{-\beta X}, \\
K_{0}^{(2)} & =\frac{1}{4 c_{0}^{2}} e^{-\alpha X}\left(e^{-\alpha L}\left[-\frac{i}{2 k_{0}(X+L)}-\frac{2 i}{k_{0} a}-2\right]+\left[\frac{i}{2 k_{0} X}+\frac{2 i}{k_{0} a}+2\right]\right), \\
K_{1}^{(2)} & =\frac{1}{4 c_{0}^{2}} e^{-\alpha(X+L)}\left[-\frac{i}{2 k_{0}(X+L)}-\frac{2 i}{k_{0} a}-2\right], \\
K_{2}^{(2)} & =\frac{1}{4 c_{0}^{2}} e^{-\beta X}\left[\frac{i}{2 k_{0} X}-\frac{2 i}{k_{0} a}+2\right], \\
K_{3}^{(2)} & =0, \\
K_{4}^{(2)} & =\frac{1}{4 c_{0}^{2}} e^{-\beta X}\left(e^{-\beta L}\left[-\frac{i}{2 k_{0}(X+L)}+\frac{2 i}{k_{0} a}-2\right]+\left[\frac{i}{2 k_{0} X}-\frac{2 i}{k_{0} a}+2\right]\right), \\
\alpha & =-2 \frac{2 i k_{0} a-1}{a}, \\
\beta & =-\frac{2}{a} .
\end{aligned}
$$

Consequently,

$$
\begin{aligned}
I_{B}(x) & =K_{0} e^{\alpha x} \text { if } x<X, \\
& =K_{1} e^{\alpha x}+K_{2} e^{\beta x}+K_{3} \text { if } X \leq x \leq X+L, \\
& =K_{4} e^{\beta x} \text { if } x>X+L,
\end{aligned}
$$

where

$$
\forall j \in \llbracket 0,4 \rrbracket, K_{j}=K_{j}^{(1)}+K_{j}^{(2)}
$$

Finally, we can compute

$$
\left\langle L_{0}^{-1} L_{1} L_{0}^{-1} L_{1}\right\rangle \phi_{0}(x)=4 c_{0}^{4} \epsilon^{2} \int G_{0}\left(x, x^{\prime}\right)\left[\left(\partial_{x^{\prime}}+i k_{0}\right)^{2} I_{B}\left(x^{\prime}\right)\right] \phi_{0}\left(x^{\prime}\right) d x^{\prime} .
$$


A Derivation of the second-order Born approximation for a slab of frozen random medium

First let's set

$$
\begin{aligned}
& K_{5}=K_{0}\left(\alpha+i k_{0}\right)^{2}, \\
& K_{6}=K_{1}\left(\alpha+i k_{0}\right)^{2}, \\
& K_{7}=K_{2}\left(\beta+i k_{0}\right)^{2}, \\
& K_{8}=-k_{0}^{2} K_{3}, \\
& K_{9}=K_{4}\left(\beta+i k_{0}\right)^{2},
\end{aligned}
$$

So

$$
\begin{aligned}
\left\langle L_{0}^{-1} L_{1} L_{0}^{-1} L_{1}\right\rangle \phi_{0}(x) & =c_{0}^{4}\left(\int_{-\infty}^{X} G_{0}\left(x, x^{\prime}\right) K_{5} e^{\alpha x^{\prime}} \phi_{0}\left(x^{\prime}\right) d x^{\prime}\right. \\
& \left.+\int_{X}^{X+L} G_{0}\left(x, x^{\prime}\right)\left(K_{6} e^{\alpha x^{\prime}}+K_{7} e^{\beta x^{\prime}}+K_{8}\right) \phi_{0}\left(x^{\prime}\right) d x^{\prime}+\int_{X+L}^{+\infty} G_{0}\left(x, x^{\prime}\right) K_{9} e^{\beta x^{\prime}} \phi_{0}\left(x^{\prime}\right) d x^{\prime}\right) \\
& =-\frac{i c_{0}^{2}}{2 k_{0}} \phi_{0}(x)\left(K_{5} I_{5}+K_{6} I_{6}+K_{7} I_{7}+K_{8} I_{8}+K_{9} I_{9}\right)
\end{aligned}
$$

Each integral $I_{i}$ is of the form

$$
\int_{l_{1}}^{l_{2}} e^{i k_{0}\left|x-x^{\prime}\right|} e^{\Omega x^{\prime}} e^{i k_{0}\left(x^{\prime}-x\right)} d x^{\prime}
$$

where $\Omega=\alpha, \beta$ or $0, l_{1}=-\infty, X$, or $X+L$ and $l_{2}=X, X+L$ or $+\infty$. Distinguishing cases where $x \in\left[l_{1}, l_{2}\right], x<l_{1}$ and $x>l_{2}$, one finds:

$$
\begin{aligned}
\left\langle L_{0}^{-1} L_{1} L_{0}^{-1} L_{1}\right\rangle \phi_{0}(x) & =-\frac{i c_{0}^{2}}{2 k_{0}} \phi_{0}(x)\left[K_{5}\left(\frac{e^{\alpha x}}{\alpha}+\frac{e^{2 i k_{0}(X-x)+\alpha X}-e^{\alpha x}}{2 i k_{0}+\alpha}\right)\right. \\
& +K_{6} \frac{e^{2 i k_{0}(X-x)+\alpha X}\left(e^{\left(2 i k_{0}+\alpha\right) L}-1\right)}{2 i k_{0}+\alpha}+K_{7} \frac{e^{2 i k_{0}(X-x)+\beta X}\left(e^{\left(2 i k_{0}+\beta\right) L}-1\right)}{2 i k_{0}+\beta} \\
& \left.+K_{8} \frac{e^{2 i k_{0}(X-x)}\left(e^{2 i k_{0} L}-1\right)}{2 i k_{0}}-K_{9} \frac{e^{2 i k_{0}(X+L-x)+\beta(X+L)}}{2 i k_{0}+\beta}\right] \text { if } x<X, \\
& =-\frac{i c_{0}^{2}}{2 k_{0}} \phi_{0}(x)\left[K_{5} \frac{e^{\alpha X}}{\alpha}+K_{6}\left(\frac{e^{\alpha x}-e^{\alpha X}}{\alpha}+\frac{e^{2 i k_{0}(X+L-x)+\alpha(X+L)}-e^{\alpha x}}{2 i k_{0}+\alpha}\right)\right. \\
& +K_{7}\left(\frac{e^{\beta x}-e^{\beta X}}{\beta}+\frac{e^{2 i k_{0}(X+L-x)+\beta(X+L)}-e^{\beta x}}{2 i k_{0}+\beta}\right)+K_{8}\left((x-X)+\frac{e^{2 i k_{0}(X+L-x)}-1}{2 i k_{0}}\right) \\
& \left.-K_{9} \frac{e^{2 i k_{0}(X+L-x)+\beta(X+L)}}{2 i k_{0}+\beta}\right] \text { if } X \leq x \leq X+L, \\
& =-\frac{i c_{0}^{2}}{2 k_{0}} \phi_{0}(x)\left[K_{5} \frac{e^{\alpha X}}{\alpha}+K_{6} \frac{e^{\alpha X}\left(e^{\alpha L}-1\right)}{\alpha}+K_{7} \frac{e^{\beta X}\left(e^{\beta L}-1\right)}{\beta}+K_{8} L\right. \\
& \left.+K_{9}\left(\frac{e^{\beta x}-e^{\beta(X+L)}}{\beta}-\frac{e^{\beta x}}{2 i k_{0}+\beta}\right)\right] \text { if } X+L<x,
\end{aligned}
$$


which is of the form

$$
\left(C_{1}+C_{2} e^{\alpha x}+C_{3} e^{\beta x}+C_{4} e^{-2 i k_{0} x}+C_{5} \frac{x}{a}\right) \phi_{0}(x)
$$

Setting $r=k_{0} a, \tilde{y}=\frac{y}{a}$ for any variable $y$ and replacing $\alpha$ and $\beta$ by their expression,

$$
\begin{aligned}
\left\langle L_{0}^{-1} L_{1} L_{0}^{-1} L_{1}\right\rangle \phi_{0}(x) & =-\frac{i\left(c_{0} a\right)^{2}}{4 r} \phi_{0}(x)\left[K_{5}\left(\frac{e^{2(1-2 i r) \tilde{x}}}{1-2 i r}+\frac{\left.e^{2(1-i r) \tilde{X}-2 i r \tilde{x}}-e^{2(1-2 i r) \tilde{x}}\right)}{1-i r}\right)\right. \\
& +K_{6} \frac{e^{2(1-i r) \tilde{X}-2 i r \tilde{x}}\left(e^{2(1-i r) \tilde{L}}-1\right)}{1-i r}+K_{7} \frac{e^{2(-1+i r) \tilde{X}-2 i r \tilde{x}}\left(e^{2(-1+i r) \tilde{L}}-1\right)}{-1+i r} \\
& \left.+K_{8} \frac{e^{2 i r(\tilde{X}-\tilde{x})}\left(e^{2 i r \tilde{L}}-1\right)}{i r}-K_{9} \frac{\left.e^{2(-1+i r)(\tilde{X}+\tilde{L})-2 i r \tilde{x}}\right]}{-1+i r}\right] \text { if } \tilde{x}<\tilde{X}, \\
& =-\frac{i\left(c_{0} a\right)^{2}}{4 r} \phi_{0}(x)\left[K_{5} \frac{e^{2(1-2 i r) \tilde{X}}}{1-2 i r}\right. \\
& +K_{6}\left(\frac{e^{2(1-2 i r) \tilde{x}}-e^{2(1-2 i r) \tilde{X}}}{1-2 i r}+\frac{e^{2(1-i r)(\tilde{X}+\tilde{L})-2 i r \tilde{x}}-e^{(1-2 i r) \tilde{x}}}{(1-i r)}\right) \\
& +K_{7}\left(-\left(e^{-2 \tilde{x}}-e^{-2 \tilde{X}}\right)+\frac{e^{2(-1+i r)(\tilde{X}+\tilde{L})-2 i r \tilde{x}}-e^{-2 \tilde{x}}}{(-1+i r)}\right) \\
& \left.+K_{8}\left(2(\tilde{x}-\tilde{X})+\frac{e^{2 i r(\tilde{X}+\tilde{L}-\tilde{x})}-1}{i r}\right)-K_{9} \frac{e^{2(-1+i r)(\tilde{X}+\tilde{L})-2 i r \tilde{x}}}{-1+i r}\right] \\
& \text { if } \tilde{X} \leq \tilde{x} \leq \tilde{X}+\tilde{L}, \\
& =-\frac{i\left(c_{0} a\right)^{2}}{4 r} \phi_{0}(x)\left[K_{5} \frac{e^{2(1-2 i r) \tilde{X}}}{1-2 i r}+K_{6} \frac{e^{2(1-2 i r) \tilde{X}}\left(e^{2(1-2 i r) \tilde{L}}-1\right)}{1-2 i r}\right. \\
& \left.-K_{7}\left(e^{-2 \tilde{X}}\left(e^{-2 \tilde{L}}-1\right)\right)+K_{8} 2 \tilde{L}+K_{9}\left(-\left(e^{-2 \tilde{x}}-e^{-2(\tilde{X}+\tilde{L})}\right)-\frac{e^{-2 \tilde{x}}}{(-1+i r)}\right)\right] \\
& \text { if } \tilde{X}+\tilde{L}<\tilde{x} .
\end{aligned}
$$

Numerically, one term appears to be dominant:

$$
\begin{aligned}
\left\langle L_{0}^{-1} L_{1} L_{0}^{-1} L_{1}\right\rangle \phi_{0}(x) & \simeq 0 \text { if } \tilde{x}<\tilde{X} \\
& \simeq-\frac{i\left(c_{0} a\right)^{2}}{2 r} \phi_{0}(x) K_{8}(\tilde{x}-\tilde{X}) \text { if } \tilde{X} \leq \tilde{x} \leq \tilde{X}+\tilde{L}, \\
& \simeq 0 \text { if } \tilde{X}+\tilde{L}<\tilde{x} .
\end{aligned}
$$


A Derivation of the second-order Born approximation for a slab of frozen random medium

Finally, we also compute

$$
\begin{aligned}
L_{0}^{-1}\left\langle L_{2}\right\rangle \phi_{0} & =\epsilon^{2}\left[-\frac{i}{2 r}\left(-\frac{e^{2 i r(\tilde{X}-\tilde{x})}}{\tilde{X}}+\frac{e^{2 i r(\tilde{X}+\tilde{L}-\tilde{x})}}{\tilde{X}+\tilde{L}}\right)+e^{2 i r(\tilde{X}-\tilde{x})}\left(1-e^{2 i r \tilde{L}}\right)\right. \\
& \left.+\frac{e^{2 i r(\tilde{X}-\tilde{x})}}{4}\left(e^{2 i r \tilde{L}}-1\right)\right] \text { if } \tilde{x}<\tilde{X} \\
& =\epsilon^{2}\left[-\frac{i}{2 r}\left(-\frac{1}{\tilde{X}}+\frac{e^{2 i r(\tilde{X}+\tilde{L}-\tilde{x})}}{\tilde{X}+\tilde{L}}\right)+\left(1-e^{2 i r(\tilde{X}+\tilde{L}-\tilde{x})}\right)\right. \\
& \left.+\frac{i r}{2}\left((\tilde{x}-\tilde{X})+\frac{e^{2 i r(\tilde{X}+\tilde{L}-\tilde{x})}-1}{2 i r}\right)\right] \text { if } \tilde{X} \leq \tilde{x} \leq \tilde{X}+\tilde{L} \\
& =\epsilon^{2}\left[-\frac{i}{2 r}\left(-\frac{1}{\tilde{X}}+\frac{1}{\tilde{X}+\tilde{L}}\right)+\frac{i r \tilde{L}}{2}\right] \text { if } \tilde{X}+\tilde{L}<\tilde{x} .
\end{aligned}
$$

Here again, one term appears to be dominant:

$$
\begin{aligned}
L_{0}^{-1}\left\langle L_{2}\right\rangle \phi_{0} & \simeq 0 \text { if } \tilde{x}<\tilde{X} \\
& \simeq \frac{\operatorname{ir\epsilon ^{2}}}{2}(\tilde{x}-\tilde{X}) \text { if } \tilde{X} \leq \tilde{x} \leq \tilde{X}+\tilde{L} \\
& \simeq 0 \text { if } \tilde{X}+\tilde{L}<\tilde{x},
\end{aligned}
$$

meaning that in the band where the medium is random:

$$
\langle\phi(x)\rangle \simeq \phi_{0}(x)\left(1+\epsilon^{2}\left[\frac{3}{2} i k_{0} a-\left(k_{0} a\right)^{2}+\frac{\left(k_{0} a\right)^{2}}{2 i k_{0} a-1}\right] \frac{x-X}{a}\right) .
$$

The corrective term is the one that appears in the Keller theory for small perturbations (2.52), although the calculation is more tedious.

Although the Keller and the Born theories are based on the same idea, the former gives directly an equation for the mean field to the second order, but requires the medium to be perturbed everywhere. On the other hand, the Born approximation gives a second-order correction to the unperturbed field, but can be computed for a localized perturbed medium and therefore accounts for boundary effects. Both theories confirm the linear increase of $\operatorname{Im}(k) / k_{0}$ in Medium 1 with frequencies in the range 1 to $5 \mathrm{mHz}$, and the approximate independence of $c_{\text {eff }}$ with respect to frequency. 


\section{Publications}

\section{Refereed publications}

- P.-L. Poulier, D. Fournier, L. Gizon and T. L. Duvall, 2020: Acoustic wave propagation through solar granulation: Validity of effective-medium theories, coda waves, A\&A, 643, A168

\section{Conference contribution}

- Waves, Instabilities and Turbulence in Astrophysical and Geophysical Flows (WITGAF 2019), Cargèse, France, July 8-19, 2019. Talk: On the validity of the frozen-in approximation for acoustic wave propagation through solar granulation.

- Second Max Planck Partner Group Workshop on Solar Physics, Mumbai, India, March 17-20, 2019. Talk: Wave propagation through convection: when is the frozen-in approximation valid?.

- XXXth General Assembly of the International Astronomical Union, Vienna, Austria, August 20-31, 2018. Poster: On the validity of the frozen-in approximation for acoustic wave propagation through solar granulation.

- Annual Meeting of the German Astronomical Society (Astronomische Gesellschaft), Göttingen, Germany, September 18-22, 2017. Poster: Multiple scattering of acoustic waves.

- Rocks \& Stars II conference, Göttingen, Germany, September 13-16, 2017. Talk: Multiple scattering of acoustic waves. 



\section{Acknowledgements}

First of all, I want to thank Laurent Gizon for giving me the opportunity to conduct my PhD research here at the Max Planck Institute for Solar System Research (MPS), which is such a great place for science and such a warm environment. His guidance throughout my $\mathrm{PhD}$ has been very valuable. All my gratitude goes to Tom Duvall for providing useful advice, entertaining conversations, and being such a great tennis partner and friend. Thanks to Olga Shishkina for her participation and advice as a member of my TAC.

Many thanks to the thesis defense committee: Laurent and Olga, and also Thorsten Hohage who kindly reviewed this thesis, Stefan Dreizler, Wolfram Kollatschny and Andreas Tilgner.

I am very grateful to Damien Fournier, without whom finishing the $\mathrm{PhD}$ would have been much more difficult. His help, patience, experience and good mood have been extremely precious. I am also indebted to Zhi-Chao Liang for being such a patient guide and teacher of data analysis. Finally, many thanks to all others on our floor, in particular Sabine, Jesper, Vincent, Dan, Aaron, Kaori, Sirila, Sarah, Ray, Robert.

I wish to thank the International Max Planck Research School for Solar System Science (IMPRS) for enabling me to pursue my doctoral studies here in Göttingen and for being such a dedicated and inspirational graduate program. Many thanks to the IMPRS coordinator Sonja Schuh for her help throughout the different phases of the $\mathrm{PhD}$. The journey also would not have been the same without the PhD students who make the MPS and the IMPRS special. I am grateful to have made incredible friends: Sebastián, Eliana, Majid, Andrea, Sihane, Mariangela, Mahmoud, Sudharshan, Felix, Bastian, Theo, Nils, and the list goes on.

Finally, I would like to thank my beloved family for their unfailing support and help, for the evenings shared on Skype, for the letters and the food deliveries (the best deliveries there are!). Special thanks to my parents: Mom, Dad, I am proud to be your son, and this thesis is a bit yours too. 